\title{
Repurposed floxacins targeting RSK4 prevent chemoresistance and metastasis in lung and bladder
}

cancer

Stelios Chrysostomou ${ }^{1^{*}}$, Rajat Roy ${ }^{1^{*}}$, Filippo Prischi ${ }^{2,13^{*}}$, Lucksamon Thamlikitkul ${ }^{1,14}$, Kathryn L Chapman $^{1,3}$, Uwais Mufti ${ }^{1}$, Robert Peach ${ }^{4,22}$, Laifeng Ding ${ }^{5}$, David Hancock ${ }^{11}$, Christopher Moore ${ }^{11}$, Miriam Molina-Arcas ${ }^{11}$, Francesco Mauri ${ }^{1}$, David J Pinato ${ }^{1}$, Joel M Abrahams ${ }^{1}$, Silvia Ottaviani ${ }^{1}$, Leandro Castellano ${ }^{1}$, Georgios Giamas ${ }^{21}$, Jennifer Pascoe ${ }^{7}$, Devmini Moonamale ${ }^{1}$, Sarah Pirrie ${ }^{8}$, Claire Gaunt $^{8}$, Lucinda Billingham ${ }^{8}$, Neil M Steven ${ }^{7}$, Michael Cullen ${ }^{7}$, David Hrouda ${ }^{9}$, Mathias Winkler ${ }^{9}$, John Post $^{18}$, Philip Cohen ${ }^{18}$, Seth J Salpeter ${ }^{15}$, Vered Bar ${ }^{15}$, Adi Zundelevich ${ }^{15}$, Shay Golan ${ }^{16}$, Dan Leibovici ${ }^{17}$, Romain Lara ${ }^{1,19}$, David R Klug ${ }^{4}$, Sophia N Yaliraki ${ }^{4}$, Mauricio Barahona ${ }^{10}$, Yulan Wang ${ }^{20}$, Julian Downward ${ }^{11}$, J Mark Skehel ${ }^{12}$, Maruf MU Ali ${ }^{13 \ddagger}$, Michael J Seckl ${ }^{1 \ddagger}$, Olivier E Pardo ${ }^{1 \ddagger}$

${ }^{1}$ Division of Cancer, Department of Surgery and Cancer, ${ }^{4}$ Department of Chemistry, ${ }^{6}$ Department of Histopathology, ${ }^{9}$ Urology, Charing Cross Hospital, ${ }^{10}$ Department of Mathematics, ${ }^{13}$ Department of Life Sciences, Imperial College London, London SW7 2AZ, UK. 'S School of Biological Sciences, University of Essex, Colchester CO4 3SQ, UK. ${ }^{3}$ Assay Biology, Domainex Ltd, Cambridge CB10 1XL, UK. ${ }^{5}$ Key Laboratory of Magnetic Resonance in Biological Systems, National Centre for Magnetic Resonance in Wuhan, State Key Laboratory of Magnetic Resonance and Atomic and Molecular Physics, Wuhan Institute of Physics and Mathematics, Chinese Academy of Sciences, Wuhan 430071 , China. ${ }^{7}$ Oncology, University Hospitals Birmingham NHS Foundation Trust, Birmingham B15 2TH, UK. ${ }^{8}$ Cancer Research UK Clinical Trials Unit, University of Birmingham, Birmingham B15 2TT, UK. ${ }^{11}$ Oncogene Biology Laboratory, The Francis Crick Institute, London NW1 1AT, UK. ${ }^{12}$ Biological Mass Spectrometry and Proteomics, MRC LMB, Cambridge CB2 0QH, UK ${ }^{14}$ Department of Medicine, Faculty of Medicine Siriraj Hospital, Mahidol University, Bangkok 10700, Thailand. ${ }^{15}$ Curesponse 6 Weizmann Street, 6423906, Tel Aviv, Israel. ${ }^{16}$ Department of Urology, Rabin Medical Center, Jabotinsky St 39, 4941492, Petah Tikva, Israel. ${ }^{17}$ Department of Urology, Kaplan Medical Center, 7610001, Rehovot, Israel. ${ }^{18} \mathrm{MRC}$ Protein Phosphorylation and Ubiquitylation Unit, School of Life Sciences, University of Dundee, Dow Street DD1 5EH. UK. ${ }^{19}$ AstraZeneca, Discovery Science, R\&D, Discovery Biology, Darwin Building, Cambridge Science Park, Milton Road, Cambridge CB4 OWG. ${ }^{20}$ Lee Kong Chian School of Medicine, Nanyang Technological University, Singapore, 636921.

${ }^{21}$ Department of Biochemistry and Biomedicine, School of Life Sciences, University of Sussex, Falmer, Brighton BN1 9QG, UK. ${ }^{22}$ Department of Neurology, University Hospital Würzburg, 97080 Würzburg, Germany.

* These authors contributed equally to the work

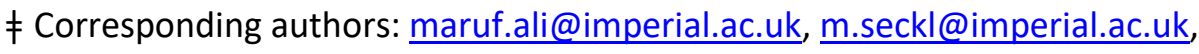
o.pardo@imperial.ac.uk

\section{Overline: CANCER}

One-sentence summary: RSK4 promotes invasiveness and drug resistance in lung and bladder cancer and inhibition of this kinase shows therapeutic potential. 
Author contributions: SC, RR, FP, LT, KLC, UM, RP, LD, DH, CM, MM-A, FM, DJP, JMA, SO, LC, GG, JP, $\mathrm{DM}, \mathrm{SP}, \mathrm{CG}, \mathrm{JP}, \mathrm{SG}, \mathrm{DL}, \mathrm{RL}, \mathrm{JMS}, \mathrm{VB}, \mathrm{AZ}$ and OEP designed and performed experiments and data analysis. $\mathrm{LB}, \mathrm{NMS}, \mathrm{MC}, \mathrm{DH}, \mathrm{MW}, \mathrm{PC}, \mathrm{SJS}, \mathrm{DRK}, \mathrm{SNY}, \mathrm{MB}, \mathrm{YW}$, JD, JMS, MMUA, MJS and OEP provided funding and performed data analysis. MJS and OEP wrote the paper.

\begin{abstract}
Lung and bladder cancers are mostly incurable due to early development of drug resistance and metastatic dissemination. Hence, better therapies that tackle these two processes are urgently needed to improve clinical outcome. We have identified RSK4 as a promoter of drug resistance and metastasis in lung and bladder cancer cells. Silencing this kinase, either through RNA interference or CRISPR, sensitised tumor cells to chemotherapy and hindered metastasis in vitro and in vivo in a tailvein injection model. Drug screening revealed several floxacin antibiotics as potent RSK4 activation inhibitors and trovafloxacin reproduced all effects of RSK4 silencing in vitro and in/ex vivo using lung cancer xenograft and genetically-engineered mouse models and bladder tumour explants. Through $X$-ray structure determination and Markov transient and Deuterium exchange analyses, we identified the allosteric binding site and revealed how this compound blocks RSK4 kinase activation through binding to an allosteric site and mimicking a kinase auto-inhibitory mechanism involving the RSK4's hydrophobic motif. Last, we show that patients undergoing chemotherapy and adhering to prophylactic levofloxacin in the large placebo-controlled randomised phase 3 SIGNIFICANT Trial had significantly $(p=0.048)$ increased long-term overall survival times. Hence, we suggest that RSK4 inhibition may represent an effective therapeutic strategy for treating lung and bladder cancer.
\end{abstract}

\title{
Introduction
}

Lung and bladder cancers are two smoking-related malignancies that are mostly incurable due to the early development of drug-resistant metastatic disease. Hence, a better understanding of the molecular mechanisms regulating these processes will help develop therapeutic strategies against these malignancies.

The p90 Ribosomal Protein S6 kinases (RPS6KAs, aka RSKs) are dual kinase domain protein kinases involved in multiple processes including protein translation, cell growth, and migration (1). Four isoforms exist in humans (RSK1-4) of which RSK1 and 2 are most studied in cancer. Their biological functions are assumed to overlap with RSK3 and 4 because these proteins have a high degree of sequence identity, especially in their $\mathrm{N}$-terminal kinase domain that mediates substrate phosphorylation. However, our previous work challenged this notion. Indeed, RSK1 differed from other isoforms in its regulation of lung cancer cell invasion as silencing of this kinase promoted metastasis in vitro (8). This suggested that the use of pan-RSK inhibitors may not be optimal for anticancer treatments (2). Moreover, RSK4 differs from other RSKs in its activation mechanism, with this isoform only requiring ERK whereas RSK1-3 need both ERK and PDK1 (1). Therefore, we hypothesise that RSK4 has divergent functions and activation patterns from other RSK isoforms. 
The role of RSK4 in cancers is controversial due to conflicting experimental data. A tumour suppressor function of RSK4 was suggested in MDA-231 breast cancer cells where RSK4 overexpression leads to cell cycle arrest, reduced clonogenic growth in vitro, and reduced tumour growth and dissemination in vivo (3). In colon carcinoma cell lines, RSK4 overexpression triggered senescence (4) consistent with large RNAi screens where RSK4 downregulation prevented p53induced senescence (5). In addition, in breast, colon and renal carcinoma, RSK4 mRNA expression was downregulated in tumour compared to normal tissues (1). In contrast, several studies in the same or different cancers suggest a tumour-promoting function for RSK4. Thus, over-expression of RSK4 enhanced whereas knockdown impaired cell migration and sunitinib resistance in renal and melanoma cell lines (6). Furthermore, RSK4 is over-expressed in breast and renal cancer at either the protein or mRNA levels (1) and in renal cancers this associated with poor patient prognosis (7). Moreover, RSK4 overexpression promoted, and silencing reduced, invasiveness of renal cell adenocarcinoma cells in vitro. One plausible explanation for these conflicting data is that RSK4 may act as a tumour suppressor or promoter in a context- or disease-dependent manner.Apart from our study demonstrating that RSK4 knockdown prevented migration and invasion of lung adenocarcinoma cells (8), the role of this kinase in lung and bladder cancer has not previously been investigated.

Here, we investigate the role of RSK4 in mediating lung and bladder cancer cell invasion as well as sensitization to chemotherapy both in vitro and in vivo through genetic manipulation (silencing and overexpression) and identify potential mechanisms to explain the effects seen. We also, screen known drugs to identify drugs or drug families which could be re-purposed as RSK4 inhibitors and crystalise part of RSK4 and undertake Markov transient and deuterium exchange analyses to assess their mechanism of action. We further test whether one of these drugs reproduces our genetic silencing data in vitro and in vivo and search for cancer patient trials that have previously used members of the drug family of interest. One such trial is found and re-analysed for whether the repurposed drug in combination with chemotherapy could prolong survival of lung and bladder plus other cancer patients. 


\section{Results}

siRNA screening reveals RSK4 as a regulator of chemosensitivity in lung and bladder cancer cells

To identify common regulators of chemo response in lung and bladder cancer, we performed a kinome siRNA screen in T24 bladder cancer cells treated with or without taxol or cisplatin and compared this to our prior screen in lung cancer cells (9). Four individual siRNA sequences targeting each kinase were used and hits selected for validation based on at least 2 sequences similarly modulating drug sensitivity (fig S1A-C). Amongst kinases found to control drug sensitivity (Fig 1A), 23 regulated the response to both cisplatin and taxol (Fig 1B, fig. S1C), and 13 of these were crossvalidated in 2 additional bladder cancer cell lines, TCCSUP and J82 (Fig 1C, fig. S1D). Cross-correlating these results with those previously-published for A549 lung adenocarcinoma cells (9) revealed RSK4 (aka RPS6KA6) as a potential common target between lung and bladder cancer. Indeed, we confirmed that silencing RSK4 sensitised both bladder and lung cancer cells to taxol and cisplatin (Fig 1D). This was reproduced in additional lung cancer cell lines (fig S2A) independently from the modality of RSK4 downregulation as two A549 clones in which RSK4 was knocked-out using CRISPR technology showed similar drug sensitisation (Fig 1E). This was associated with potentiation of apoptosis and increased DNA damage as assessed by caspase 3/7 activation (Fig 1F) and elevated phospho-H2AX nuclear foci (Fig 1G), respectively. However, RSK4-silenced cells did not show decreased cell viability in the absence of drug (Fig 1D), despite increased total caspase 7 expression, as well as cleavage of caspase 7 and PARP (Fig $\mathbf{1 H}$, fig. S2B), suggesting that RSK4-knockdown only primes the apoptotic process. Conversely, RSK4 overexpression made A549 cells chemoresistant (fig S2C), with decreased background caspase activation and PARP cleavage (fig S2D). The increased caspase 3/7 activity in RSK4-downregulated cells was rescued by RSK4 overexpression (fig S2E). In multiple NSCLC and T24 cell lines, sensitisation to chemotherapy was associated with decreased expression of the anti-apoptotic proteins BCL2, CIAP1 and CIAP2 that we previously showed promote chemo-resistance in lung cancer (Fig 11, fig. S2F) (10). This decrease occurred at the transcriptional level (Fig 1J). Similar decreases in CIAP1 and cIAP2, but not BCL2, occurred in A549 RSK4-CRISPR clones (fig S2G), which may indicate that cIAP changes are more relevant to chemosensitisation in this biological context. Moreover, cIAP2 is likely the major mediator of the RSK4-induced chemoresistance as only cIAP2 mRNA and protein expression were increased by RSK4 overexpression in A549 cells (fig S2H-I) and clAP2 but not clAP1 rescued chemosensitisation following RSK4 silencing (fig S2J). In short, RSK4 downregulation promotes cell death in several lung and bladder cancer cell lines treated with two clinically relevant chemotherapeutic compounds.

\section{RSK4 controls the migration and invasion of lung and bladder cancer cells}

We previously published that RSK4 silencing decreased the migration and invasion of A549 cells (8). Similarly, RSK4 knockdown hindered the migration and invasion of bladder and lung cancer cell lines (Fig 2A, 2B, fig. S3A), whereas RSK4 overexpression promoted migration of A549 cells (fig S3B). Microscopic image analysis of RSK4-silenced cells revealed increased cell clustering and roundness (Fig. 2C, fig. S3C), suggesting a reversal of epithelial-mesenchymal transition (EMT). In agreement, analysis of lysates from RSK4-silenced and control cells for EMT markers revealed decreased vimentin and $\mathrm{N}$-cadherin and increased epithelial marker E-cadherin (Fig. 2D-left, fig. S3D-E). 
Conversely, RSK4 overexpression had the opposite effect (Fig. 2D-right, fig. S3F). The decreased cell migration observed after siRNA-mediated RSK4 silencing was reproduced in RSK4-CRISPR A549 cells and rescued by RSK4 overexpression, demonstrating the RSK4-selectivity of this phenotype (fig. S3G). EMT is partly controlled by NFKB (11) and reduced expression of EMT markers after RSK4 downregulation associated with decreased NFKB activity (Fig. 2E, fig. S3H) and decreased RELA nuclear localisation (Fig. 2F). This suggested that NFKB activity is controlled by RSK4. RELA nuclear accumulation is negatively regulated through acetylation on K122 and K123 by the acetyl-transferase p300 (12). Figure 2 G shows that Ser89 phosphorylation of p300, an event that inhibits p300's acetyltransferase activity (13), was decreased in RSK4-silenced A549 and T24 cells. Consequently, RSK4 may regulate the activity of the NFKB pathway by direct phosphorylation of $\mathrm{p} 300$, as recombinant RSK4 phosphorylates p300 on Ser89 in vitro (Fig. $2 \mathrm{H}$ ). The role of NFkB in RSK4's effects on EMT markers and cell migration were confirmed by active-RELA overexpression reverting the changes in EMT markers and decreased cell migration in RSK4-downregulated A549 cells (fig. S4A-B). In contrast, RSK4-induced chemo-resistance was not mediated by RELA, as active-RELA overexpression failed to prevent drug sensitisation or decreased cIAP2 expression in RSK4-downregulated cells (fig. S4C-E). Although changes in cell migration and the actin cytoskeleton play important roles in cellular invasion, resistance to anoikis is crucial to metastatic dissemination, as this maximises the survival of circulating cancer cells (14). Figure $2 \mathrm{I}$ shows that unlike RSK1-silenced or non-targeted cells, RSK4downregulated A549 and T24 cells underwent cell death when placed in suspension for $18 \mathrm{~h}$. Taken together, our data suggest that RSK4 silencing impairs the metastatic program of lung and bladder cancer cells.

\section{RSK4 is overexpressed in lung cancer and correlates with decreased survival in patients with adenocarcinoma}

To assess the potential clinical relevance of our findings, we analysed tissue microarrays of normal and lung cancer tissue samples for RSK4 protein expression. Staining optimisation using paraffinembedded A549 cells silenced or not for RSK4 demonstrated that immunoreactivity depended on RSK4 expression, therefore confirming the antibody's specificity (fig. S5A), similarly to that observed in Western blotting (fig. S5B). Figure 3A shows that whereas RSK4 was undetectable in all normal lung specimens, $57 \%$ of primary lung cancers $(n=97)$ were positive for this kinase. Western blotting of NSCLC and normal lung epithelial cell lines showed similar differences, suggesting that RSK4 expression in our cell lines was representative of that seen in the clinic (fig. S5C). Stratifying our clinical samples for lung cancer subtypes showed that the frequency and intensity of RSK4 expression was highest in adenocarcinoma (AdCa), decreased in squamous cell (SCC) NSCLC and lowest in small cell lung cancers (SCLC) (Fig. 3B-C). Similar differences were observed at mRNA level in TCGA datasets where lung AdCa, but not SCCs showed a significant ( $\mathrm{p}=3.27 \mathrm{e}-06)$ increase in $R S K 4$ expression over normal lung (Fig. 3D). To test whether RSK4 was important in lung AdCa, we analysed publically available lung cancer survival data. When all lung cancer subtypes were considered, high RSK4 mRNA expression associated with poorer prognosis (fig. S5D). However, when subtypes were separated, high-expressing AdCa showed significant decreased overall survival (OS) $\left(p=9.610^{-6}\right)$ whereas survival of patients with SCC was unaffected (Fig. 3E). This association between RSK4 expression and OS existed despite RSK4 expression being far lower than other RSK isoforms across all lung cancers or AdCa alone (fig. S5E). Amongst other RSKs, only RSK3 showed increased mRNA expression in AdCa compared to normal lung samples, whereas RSK2 showed decreased and 
RSK1 unchanged expression (fig. S5F). Nevertheless, a negative association between RSK expression and survival was only observed for RSK4 as similar analysis for other RSK isoforms showed that high expression either did not impact (RSK1) or was associated with improved OS (RSK2 and RSK3) in patients with AdCa (fig. S5G). Similar to the case of lung AdCa, analysis of bladder cancer samples showed lower RSK4 mRNA expression as compared to other RSKs (fig. S6A), and RSK4 overexpresion in cancer as compared to normal bladder samples (fig. S6B). Last, consistent with our in vitro data showing that RSK4 mediates the invasiveness of lung AdCa cells, we found that $52 \%$ of AdCa metastatic lesions expressed more RSK4 protein than their corresponding primary tumours, whereas this number fell to $24 \%$ in SCC (Fig. 3F). These results are also consistent with the difference in correlation between OS and RSK4 expression in patients with lung AdCa and SCC. Taken together, our data suggest that RSK4 over-expression as a predictor of survival might be most relevant in lung AdCa.

\section{RSK4 silencing impairs tumour progression and maximises therapy response in vivo}

We next assessed the effects of RSK4 silencing on tumour growth in vivo using A549 non-targeting or RSK4-CRISPR cells injected subcutaneously into nude mice. Tumour growth was noticeably delayed for both RSK4-CRISPR clones tested as compared to their non-targeted counterpart (Fig. 4A). Moreover, potentiation of cisplatin response was seen for RSK4-CRISPR as compared to nontargeted tumours (Fig. 4A-B). Because RSK4 silencing imparied cell invasion and anoikis in vitro, we also assessed metastasis in vivo. RSK4-CRISPR or non-targeted A549 cells were injected into the tail vein of Severe Combined Immunodeficiency (SCID) mice and their lungs histologically examined 2 months later. This revealed a decreased ability of RSK4-CRISPR cells to colonise the lungs of injected mice (Fig 4C, fig. S7). Hence, RSK4 downregulation hinders lung adenocarcinoma tumour growth and dissemination in vivo.

\section{Small compound library screening identifies moxifloxacin as a RSK4 activation inhibitor}

Our data suggested that RSK4 inhibition may be clinically beneficial in the treatment of lung adenocarcinoma. Although pan-RSK inhibitors exist (15) (16), we previously published that RSK1 downregulation promotes lung cancer invasiveness (8). Here, we reproduce this finding by treating A549 cells with the pan-RSK inhibitor SL-0101 (fig. S8A). Moreover, contrary to the results obtained for RSK4, RSK1 silencing in bladder and lung cancer cells induced resistance to taxol and cisplatin (fig. S8B) and increased clAP2 expression (fig. S8C). Last, unlike RSK4, RSK1 was well expressed in normal lung epithelial cells as aforementioned. Hence, inhibiting this kinase as part of therapeutic targeting of RSK4 may yield distinct toxicity and loss of anti-cancer effects. Consequently, we wished to identify selective RSK4 inhibitors. We therefore developed a high-throughput Homogeneous TimeResolved Fluorescence (HTRF)-based assay to identify molecules interfering with RSK4 activation by ERK as, unlike other RSKs, RSK4 does not require PDK1 (1). Because bacterially-produced recombinant RSK4 showed high basal activity in vitro, we first inactivated it through dephosphorylation with the phosphatase PP1MA before incubation with test compounds and subsequent re-activation by ERK2 (Fig. 5A, fig. S8D). This favoured the selection of molecules likely to be non-ATP competitive. We screened 1675 molecules and discovered twelve compounds showing $>75 \%$ inhibition of RSK4 activation at $30 \mu \mathrm{M}$, amongst which was the antibiotic, 
moxifloxacin (Fig. 5B). This molecule acted as a micromolar $\left(\mathrm{IC}_{50}=6.2 \mu \mathrm{M}\right)$ non-ATP competitive reversible inhibitor of RSK4 activation (fig. S8E-F) and did not interfere with ERK activity in contrast to the well documented ATP-competitive pan-RSK inhibitor, BI-D1870 (fig. S8G). Increasing the concentration of RSK4 by 10 fold did not modify the efficiency of moxifloxacin, suggesting it does not act by degrading RSK4 (fig. S8E). Time-dependence inhibition studies showed a linear relationship with product formation in the presence of moxifloxacin or BI- D1870 (fig. S8H), indicating that moxifloxacin is not a slow binder and that mechanism of action studies could be performed using classical steady-state analysis. Michaelis Menten kinetics confirmed that in the presence of submaximal inhibition by BI-D1870, RSK4 maintained ATP Vmax but showed a trend to increased Km in agreement with the ATP competitive nature of this inhibitor (fig. S8J-K). In contrast, when incubated with moxifloxacin, RSK4 maintained Km and displayed reduced $\operatorname{Vmax}(\alpha=1)$, confirming ATP noncompetitive binding equally to the Enzyme and Enzyme-Substrate state of the enzyme (fig. S8I-K). Moreover, Moxifloxacin showed a preference for preventing RSK4 activation rather than inhibiting fully-active RSK4 and further favoured RSK4 over RSK1 (fig. S8L), making this compound a useful chemical starting point for future synthesis of RSK4-selective inhibitors.

\section{Trovafloxacin reproduces the effects of RSK4 silencing in vitro}

Following our results with moxifloxacin, we screened other floxacin family members in our RSK4 HTRF-activation assay and identified both additional inhibitors (trovafloxacin, marbofloxacin, rufloxacin, tosufloxacin) as well as inactive analogues (ciprofloxacin, danofloxacin, and piromidic acid) (Fig. 5B, fig. S9A). As all these compounds are active antibiotics, we assumed that their RSK4targeting activity was independent of their known clinical indication. However, no clear structureactivity relationship was readily identifiable (fig. S10). Crucially, only the compounds active against RSK4 sensitised A549 cells to cisplatin (Fig. 5C, fig. S11A). In particular, trovafloxacin showed superior abilities to moxifloxacin in this assay with $7.5 \mu \mathrm{M}$ of this compound reducing the $\mathrm{IC}_{50}$ for cisplatin (Fig. 5C). This relationship was synergistic (fig. S11B) and the potentiation of cisplatin or taxol effects maximal when combined with $\approx 10 \mu \mathrm{M}$ trovafloxacin (fig. S11B-C). This correlated with promotion of caspase activation in response to drug treatment in A549 cells (Fig. 5D), an effect dependent on the expression of RSK4. Indeed, RSK4 silencing using a 5'UTR-targeting siRNA prevented potentiation of caspase activation by trovafloxacin over that obtained with cisplatin alone whereas simultaneous RSK4 overexpression rescued this difference (Fig. 5E). In contrast, the panRSK inhibitor BI-D1870 failed to promote cisplatin-induced caspase activation (Fig. 5E). As with RSK4 silencing, increased apoptosis in response to trovafloxacin associated with decreased expression of BCL2 and CIAP1/2, an effect not seen with either ciprofloxacin (non-RSK4 inhibitor) or BI-D1870 (Fig 5F, fig. S11D). Moreover, trovafloxacin treatment reproduced the inhibition of cell migration/invasiveness (Fig. 5G-H, fig. S11E-F) and EMT reversal (fig. S11G) previously observed with RSK4 silencing in A549 cells. Other RSK4-targeting floxacins (moxifloxacin, tosufloxacin) shared this ability, whereas non RSK4-targeting compounds (ciprofloxacin, danofloxacin) did not impair cell invasiveness (Fig. $\mathbf{5 H}$ ). In addition, differential scanning fluorimetry assays demonstrated that trovafloxacin bound the inactive/dephosphorylated, but not the fully-active, RSK4 N-terminal kinase domain (RSK4-NTKD) (Fig. 5I), supporting the selectivity profile previously seen for moxifloxacin. In contrast, ciprofloxacin failed to bind RSK4 in the same assay (fig. S11H), explaining why this compound fails to inhibit this kinase. Last, we tested $10 \mu \mathrm{M}$ trovafloxacin, corresponding to the $\mathrm{IC}_{50}$ of this drug on recombinant RSK4, against a panel of 140 kinases in an in-vitro activity screen. No 
other kinase was substantially inhibited by trovafloxacin at this concentration (Supplementary Excel data file). In short, trovafloxacin appears to be a reasonably-selective allosteric RSK4-NTKD activation inhibitor.

\section{RSK4-NTKD crystal structures}

To obtain structural and mechanistic information on RSK4 and elucidate how it might be allosterically regulated, we solved the crystal structures of the phosphorylated (pS232) and dephosphorylated RSK4-NTKD, as well as the S232E phospho-mimetic mutant (table S1, fig. S12A-C). All three structures display a classic bilobal protein kinase fold (Fig. 6A), with the activation loop, which runs between the $\mathrm{C}$ - and $\mathrm{N}$-lobes, well-ordered and visible in dephosphorylated and pS232 RSK4-NTKD structures. The overall folding of these NTKD versions is identical, with an RMSD of 0.35 $\AA$ between RSK4 NTKD chain A and RSK4 NTKD S232E chain A, and 0.33 Å between RSK4 NTKD chain $A$ and RSK4 NTKD pS232 chain A.The two molecules in the asymmetric unit of the RSK4 NTKD pS232 are in the same orientation seen for the dephosphorylated protein, with only $2.6 \AA$ translation of chain $B$ in respect to its relative position in RSK4 NTKD when using chain $A$ as reference. During the refinement stage of RSK4 NTKD pS232, we found a well-defined electron density on the activation loop of chain A in which it was possible to model the phosphate of phosphorylated S232 (fig. S12A). The phosphorylation is oriented inward and points towards D197, part of the HRD motif, and, at the same time, the side chain of D197 moves $1.3 \AA$ closer to the activation loop compared to its relative position on the dephosphorylated structure.

In all three structures the top part of the C-helix, present in classical protein kinase topology (17), is disordered with the bottom part forming a helix that swings away from its classical position in PKA by up to $12 \AA$ (Fig. $6 \mathrm{~B}$ ). In this new position, the $\mathrm{C}$-helix is not involved in any catalytic activities. In its place lies the $\beta B$-sheet formed by $\beta 1-\beta 5-\beta 10$ (residues: $52-56,109-115,220-222$ ). The $\beta B$-sheet has been previously seen in both MSK1- and RSK2-NTKD crystal structures $(18,19)$, suggesting that this motif may characterise AGC family members with two kinase domains on the same polypeptide chain. Furthermore, in agreement with the RSK2-NTKD structure (18), our data suggest that the $\beta$ Bsheet is a structural feature of both inactive and active states. This was confirmed by far UV CD spectra of the three proteins, which are characteristic of a mixed $\alpha \beta$ secondary structure content and suggest the absence of major conformational changes between inactive and active states (fig. S12A, fig. S12D). This absence may facilitate RSK4 activation, as it would lower the energy barrier to reach the active state and is consistent with the high autophosphorylation property of RSK4 and with evidences that basal cellular kinase activity of ERK1/2 can already activate RSK4 to 30-50 \% (20). The three structures have similar active site organisation and AMP-PNP coordination (fig. S12C). Specifically, the N1 and N6 of the AMP-PNP adenine ring form hydrogen bonds with the backbone of L155 and of D153, as well as hydrophobic interactions with L205, A103, V87 and L79. The position of the $\alpha$-and $\beta$-phosphate groups is stabilized by interactions with the side chains of the conserved residues $\mathrm{K} 105, \mathrm{~N} 203, \mathrm{~K} 221, \mathrm{~T} 215$ and $\mathrm{F} 84$. The $\gamma$-phosphate interacts with $\mathrm{K} 200$, which is indispensable for the formation of enzymatic intermediate during catalysis. The AMP-PNP phosphates are in an optimal alignment for transferring the $\psi$-phosphate to the substrate (17) in all three structures. The major difference in the active sites as compared to a typical protein kinase is the contribution of residues from the $\beta B$-sheet motif, particularly K221. The residue K221 extends down from $\beta 10$ of the $\beta B$-sheet, and inserts directly into the active site, interacting with D216 (part of the DFG motif) and with the $\beta$-phosphate of the AMP-PNP and the three together should 
coordinate $\mathrm{Mg}^{++}$ion (due to resolutions limits, we have not been able to confidently place $\mathrm{Mg}^{++}$ ions in the structure), which help the correct positioning required for ATP binding and catalysis (17). Furthermore, K221 produces an interaction network similar to that of K72 in PKA, known to be important in catalysis. This arrangement is almost identical to the active site of RSK2 NTKD (18) (fig. S12C), where mutations of K216, equivalent of K221 in RSK4, caused impaired RSK2 NTKD activity (18). Taken together, these structural data strongly confirm the importance of K221 for RSK4 function.

A unique feature of dephosphorylated and pS232 RSK4-NTKD structures is the presence of a second AMP-PNP molecule which could be modelled with full occupancy (Fig. 6C). This AMP-PNP contacts a symmetry equivalent molecule and is important for crystal lattice formation. It is located in a surface area absent in classical AGC kinases, created through structural rearrangement caused by formation of the $\beta B$-sheet and the displacement of the C-helix. The AMP-PNP molecule in the secondary site is stabilised by a network of hydrogen bonds with water molecules and the side chains of residues C234, H250, Q81, K86, R247, D225 and K113. C234 and H250 together with the $\beta$ - and $\psi$-phosphates coordinate in a "Zing Finger"-like way a $\mathrm{Zn}^{++}$ion. This tetrahedral coordination clearly distinguishes this $\mathrm{Zn}^{++}$ion from a $\mathrm{Mg}^{++}$ion. This coordination is identical in the dephosphorylated and pS232 RSK4NTKD structures. We speculated that this unique surface is a "hot spot" (21) and represents a promising target for drug design.

\section{RSK4 hydrophobic motif interaction site}

Many AGC kinases possess a hydrophobic motif (HM), located C-terminal of the kinase domain, that is phosphorylated to achieve maximal activity (22). In classic AGC kinases the HM wraps around the $\mathrm{N}$-lobe, where a phosphate-binding pocket is localized, and stabilizes the active conformation of the C helix (23) (24). For RSK1-3, phosphorylation of the HM - found within the linker region - acts to recruit PDK1, which then phosphorylates the NTKD activation loop, resulting in activation of these RSKs. However, because RSK4 activation is PDK1-independent (20), we investigated the role of the $\mathrm{HM}$ in this process. Due to the presence of the $\beta B$-sheet the phosphate-binding pocket of RSK4NTKD is rotated $\approx 50^{\circ}$ clockwise compared to that of other AGC kinases. Moreover, this pocket is where we located the second AMP-PNP molecule (Fig. 6C). We used this information to guide a restrained docking simulation (25) using our RSK4-NTKD structure and a fully flexible phosphorylated peptide encompassing the HM sequence (NAHQLFKGF-pS-FVATSIAEE). HADDOCK (25) produced two clusters of solutions which represents $98.5 \%$ of the water-refined models the software generated and the best one had a score of $-189.0+/-18.2$, a cluster size of 190 with RMSD from the overall lowest-energy structure of $1.5+/-1.2 \AA$ and a $Z$ score of -1 . The most striking feature of the model generated by HADDOCK (26) is the position of the pS389 that occupies the same area of the $\gamma$ phosphate of the second AMP-PNP molecule, supporting our hypothesis that the latter is mimicking the HM (Fig. 6D). Specifically, the pHM position is mainly stabilised by a large network of hydrophobic interaction, while the pSer 389 forms H-bonds with E54, K113, C234 and R247. Taken together, our bioinformatics studies identified a potential phosphate-binding pocket in RSK4-NTKD.

\section{Mathematical models based on the RSK4-NTKD structure predict an allosteric binding site}


To obtain structural information about trovafloxacin binding to RSK4 we tried soaking and cocrystallisation experiments using both RSK4-NTKD and RSK4-NTKD S232E. Resulting crystals either did not contain trovafloxacin or failed to diffract. Consequently, as our wet-lab experiments suggested that trovafloxacin is an allosteric inhibitor, we adopted Markov Transient Analysis (MTA). This atomistic graph-theoretical approach can identify allosteric hotspots on the RSK4-NTKD without any a priori knowledge other than the location of the active site. MTA quantifies the speed at which a random walk diffuses on the atomistic protein graph constructed from interatomic strong and weak chemical interactions by computing the half-time $t 1 / 2$ for each atom (27). We applied MTA to our RSK4-NTKD structure to establish the specific pattern of propagation of perturbations originating from the active site and diffusing into the rest of the protein (Fig. 6F). Using quantile regression (28) (29) on the computed $t_{1 / 2}$, MTA identified a statistically significant hotspot (residues with $\leq 5 \%$ significance level) that corresponded to the second AMP-PNP binding site (Fig. 6G). The identified allosteric site exhibited a direct communication pathway with the active site (Fig. 6F), as confirmed in more detail through exhaustive computational alanine screening (fig. S13A-B). Each residue was individually alanized computationally to identify mutations that perturbed the communication between the active and obtained allosteric sites. Significant residues (one-sided, $Z>1.64, p<0.05$, red) under alanisation (fig. S14A) formed one of two different pathways connecting the active and allosteric sites (fig. S14B). Taken together, our data suggest that the second AMP-PNP binding site represents an allosteric site on the RSK4-NTKD.

\section{Trovafloxacin docking and impact on Markov transient propagation}

We performed molecular docking of Trovafloxacin using AutoDock Vina (30) around the site where the second AMP-PNP molecule sits in our crystal structures. This suggested that trovafloxacin bound to that site with a favourable energy of $-8.1 \mathrm{kcal} / \mathrm{mol}$ (Fig. 6E), which corresponded well with our in vitro inhibition data. We noticed that trovafloxacin and the allosteric inhibitor positive controls sarafloxacin and tosufloxacin tosylate had a similar overall architecture and were predicted to bind RSK4 in a similar way (fig. S13C). In contrast, the non-inhibitor negative controls ciprofloxacin and clinafloxacin, which have a more liner structure, occupied a slightly different site on RSK4 (fig. S13D). This may be connected to the predicted 1.5 order of magnitude difference in the binding energy between RSK4 allosteric inhibitors $(-8.3$ and $-8.4 \mathrm{kcal} / \mathrm{mol}$ for sarafloxacin and tosufloxacin tosylate, respectively) and non-inhibitors ( -6.6 and $-6.7 \mathrm{kcal} / \mathrm{mol}$ for ciprofloxacin and clinafloxacin, respectively). Comparison of the trovafloxacin docking model with the RSK4-NTKD X-ray structure showed that the naphthyridine and phenyl moieties of trovafloxacin occupied the same cavity where the adenine ring of the second AMP-PNP molecule sits, whereas the azabicyclo group pointed outwards and occupied the same area as the ribose and $\alpha$-phosphate of the AMP-PNP (Fig. $6 \mathrm{C}$ and E). To further validate this binding pose, we repeated the molecular docking using alternative software, GLIDE (31). The top-ranked pose, nearly identical to the one obtained using AutoDock Vina (fig. S13E), had a binding free energy (MM-GBSA $\Delta \mathrm{G}_{\text {bind }}$ ) of $-31.93 \mathrm{kcal} / \mathrm{mol}$.

To assess the impact of trovafloxacin binding to the second AMP-PNP site, we again employed MTA to test the reverse propagation of perturbations originating from the allosteric site into the rest of the protein in the presence or absence of AMP-PNP and our inhibitor. Despite the close similarity of the binding for the two molecules, MTA revealed a marked change in the communication pathways originating from the trovafloxacin-binding site as compared to those originating from the AMP-PNP- 
binding site (Fig. $6 \mathrm{H})$. In particular, MTA revealed enhanced communication from the trovafloxacin site to the active site, whereas propagation from the AMP-PNP site displayed direct communication with the activation loop and lower lobe of RSK4 (fig. S13F). These data suggest that the binding of trovafloxacin would impact the active site, providing a rational for the activity of this compound on RSK4 activation.

\section{Determination of trovafloxacin's binding site on RSK4 through hydrogen/deuterium exchange}

To gain further insight into the mechanism of action of trovafloxacin, we carried out hydrogen/deuterium exchange studies with RSK4 in the presence or absence of the drug. In this analysis, the protein is exposed to deuteriated water $\left(\mathrm{D}_{2} \mathrm{O}\right)$, which allows hydrogens on the protein backbone amides to be exchanged for deuterium. The exchange is stopped at various times by reducing the $\mathrm{pH}$, the protein is digested with pepsin, and the deuterium content of peptides determined by mass spectrometry (32). Because the rate of exchange is dependent on the tertiary structure (degree of hydrogen bonding) and solvent accessibility of amide hydrogens, such studies can yield information about binding or protein conformation, which are revealed by changes in the kinetics of deuterium incorporation into specific amino acids/peptides (33) (34).

Figure $6 \mathbf{I}$ shows a difference plot in daltons of each RSK4 peptide induced by the presence of trovafloxacin, averaged over all replicates and time points. Three regions, each covered by peptic peptides (fig. S14A), showed a reduction in exchange. One of these regions (533-552) was towards the NTKD's C-terminus, whereas a second was adjacent to this in the NTKD kinase C-terminal region (627-636). A third region (246-279) N-terminal to the NTKD also showed reduced uptake of deuterium, but was outside of any predicted domain. The butterfly plot indicates that all three sites showing reduced uptake are in regions of sequence that are more exposed to bulk solvent. From the uptake kinetics these sites are predicted to be unstructured loops rather than $\alpha$-helices or $\beta$-sheets (fig. S14B). The positions of protection suggest that trovafloxacin is not binding directly in the active or nucleotide binding sites of RSK4, but instead has an allosteric effect, inducing a conformational change in the overall structure of RSK4. The overlap between the docking data, the Markov transient analysis and the deuterium exchange results suggest that the 627-636 stretch in our tagged protein (aa 398-407 on RSK4) corresponds to the region of trovafloxacin binding, whereas the other protected sites undergo subsequent conformational changes as a result of the allosteric nature of this region. Other minor differences observed were close to the background error of the system and therefore were considered non-significant.

\section{Trovafloxacin and the role of the HM in RSK4 activation}

Our data suggest that both trovafloxacin and the HM interact with the second AMP-PNP binding site on the RSK4-NTKD (Fig. 6C-E). As MTA predicts this region is an allosteric site, we hypothesized that the HM may affect the autophosphorylation rate of RSK4-NTKD. To test this hypothesis, the RSK4NTKD was incubated in presence of saturating amount of ATP/ $\mathrm{Mg}^{2+}$ alone or with the HM synthetic peptides HP (NAHQLFKGFSFVATSIAEE) or pHP (NAHQLFKGF-pS-FVATSIAEE) corresponding to residues 380-398 of RSK4 (fig. S15). RSK4-NTKD autophosphorylation was measured over time using a phospho-Ser232-specific antibody. Incubation with pHP promoted RSK4-NTKD autophosphorylation compared to that of the HP or the RSK4-NTKD alone (fig. S15). Taken together, 
our data suggest that the phosphorylated HM binds RSK4-NTKD to increase its autophosphorylation rate. This explains how trovafloxacin inhibits RSK4 activation through preventing HM docking to the phosphate-binding pocket of the kinase.

\section{Trovafloxacin inhibits tumour growth and sensitises tumours to drug treatment in vivo}

Having shown how trovofloxacin might mechanistically impair RSK4 activation and that it reproduces the biological effects of RSK4 downregulation in vitro, we assessed the activity of this drug in vivo. Luciferase-encoding A549 cells (A549-Luc) were injected subcutaneously into nude mice. Once tumours became palpable, the mice were randomised to receive or not daily oral gavage of trovafloxacin (30 mg/kg) and weekly intraperitoneal injections of cisplatin $(5 \mathrm{mg} / \mathrm{kg})$ for 14 days. Bioluminescent whole-animal imaging performed on the first and last days of treatment after injection of luciferin (Fig. 7A, fig. S16A) shows that, as for RSK4 CRISPR cells, administration of trovafloxacin significantly $(p<0.05)$ decreased tumour growth compared to vehicle-only treatment and the effect of the trovafloxacin/cisplatin combination was superior to that of cisplatin alone $(p<0.01)$. This potentiation of cisplatin response was consistent with the statistically significant increase in caspase 3 cleavage observed in tumour samples from animals treated with the drug combination as compared to trovafloxacin $(p<0.001)$ or cisplatin alone $(p<0.01)$ (Fig. 7B, fig. S16B). The ability of trovafloxacin to potentiate response to cisplatin was further confirmed in the $\mathrm{KRAS}^{\mathrm{V} 12} / \mathrm{TP}^{-{ }^{--}}$(KP)-driven genetically-engineered mouse model where spontaneous lung adenocarcinoma tumours are triggered through adenoviral Cre-recombinase delivery (35) (Fig. 7C-F). This was consistent with trovafloxacin-induced molecular changes in cells isolated from KP tumours, such as decreased cIAP2 expression and increased caspase 3 cleavage, similar to those seen in A549 cells (Fig. 7C, fig. S16C), whereas the RSK4 non-targeting compounds ciprofoxacin failed to elicit such changes. The trovafloxacin/cisplatin combination was associated with decreased tumour number and volume as assessed by macroscopic observation (Fig. 7D) and by $\mu \mathrm{CT}$ scanning (Fig. 7E-F, fig. S16D, movie S1 and S2). For bladder cancer, as we lacked a genetically engineered mouse model, we instead employed a recently described human cancer explant system (36). We demonstrated that the cisplatin/trovafloxacin combination was beneficial in 3 out of 4 patient-derived bladder cancer explants, with synergistic effects observed in two and additive effects in one (fig. S16E). The last patient's tumour showed extreme sensitivity to cisplatin alone so the added benefit of trovafloxacin could not be assessed. Collectively, our results suggest that administration of RSK4-targeting floxacins may provide clinical benefit in patients with lung adenocarcinoma and bladder cancer.

\section{Levofloxacin inhibits RSK4 in vitro and prolongs survival of patients with cancer}

Because floxacins are frequently used in the clinic to prevent infections in patients undergoing chemotherapy we wondered whether this could be associated with improved OS. A large placebocontrolled randomised phase 3 study (SIGNIFICANT Trial) (fig. S17) demonstrated that levofloxacin reduced infective complications in patients undergoing chemotherapy in several cancer types, including lung and bladder cancer (37). HTRF assays revealed that levofloxacin inhibited RSK4 activation, resulting in decreased cellular CIAP1 and cIAP2 expression (fig. S18A-B) at concentrations achievable from the dose given in the clinical trial $(38,39)$. We therefore undertook a retrospective exploratory analysis of 1565 SIGNIFICANT trial patients to investigate whether the use of 
prophylactic levofloxacin during chemotherapy treatment had an effect on long-term OS. In patients adhering to trial treatment ( $n=937$ ), OS was significantly longer for those taking levofloxacin compared to placebo (median OS 72 versus 59 months, respectively, HR 0.83; 95\% $\mathrm{Cl} 0.69-0.999 ; \mathrm{p}$ $=0.048$; fig S18C-left). Analysis of non-adherent patients $(n=543)$ further substantiated this levofloxacin effect by showing no difference in OS (HR=1.11; 95\% $\mathrm{Cl} 0.89-1.40 ; \mathrm{p}=0.36$; fig S18Cright). Sensitivity analysis showed that excluding patients who died during treatment did not change the conclusions and thus this improvement in OS could not be due to a reduction in infective deaths due to the antibacterial effect of levofloxacin. The hazard ratio was further improved for lung and bladder cancer patients compared to the entire cohort (fig. S18D). Collectively, these results provide intriguing clinical evidence that a RSK4-targeting floxacin might also potentiate the effects of anticancer treatments and improve survival particularly in patients with lung or bladder cancer.

\section{Discussion}

Our findings demonstrate the role of RSK4 as a tumour promoter in lung adenocarcinoma and bladder cancer cells. Overexpression of this kinase lead to drug resistance and increased cell migration in lung and bladder cancer cell lines in vitro, and correlated with poorer survival in lung adenocarcinoma patients. Conversely, silencing this kinase using RNA interference or CRISPRmediated knock-out, sensitised cells to chemotherapeutic drugs, and prevented invasion in vitro and in vivo. Our data were consistent with findings in renal carcinoma $(6,7)$, but conflicted with the proposed role of RSK4 as a tumour suppressor in breast cancer $(3,4,40)$. Several explanations might account for this discrepancy including a tissue-specific role of RSK4 or differential expression of RSK4 splice variants as at least two protein-coding transcripts for RSK4 are reported in Ensembl; RPS6KA6201 and RPS6KA6-204. Further work is necessary to elucidate these seemingly conflicting tissuespecific data.

Our observations led us to hypothesize that a selective RSK4 inhibitor might represent a valuable therapeutic tool in the treatment of lung and bladder cancer. Indeed, RSK4 protein is overexpressed in the majority of lung malignancies, especially in adenocarcinoma, but undetectable in normal lung and poorly expressed in most other tissues (1). Similarly, bladder cancer samples show increased RSK4 mRNA expression compared to matching normal tissue. Hence, RSK4 inhibitors could exhibit some degree of specificity for these cancers. Moreover, the fact that RSK1, unlike RSK4, is well expressed in a variety of normal human tissues (1) may suggest that a RSK4-selective, rather than pan-RSK, inhibitor would be preferable. In support of this, our previous research showed that RSK1 silencing promoted invasion of lung cancer cells (8), an effect also seen in A549 cells with the panRSK inhibitor SL0101 but not the pan-RSK inhibitor BI-D1870 (41). Furthermore, we showed here that RSK1 in contrast to RSK4 silencing rendered lung and bladder cancer cell lines chemo-resistant. $\mathrm{S}$, while silencing RSK2 or RSK3 either led to inconsistent biological outcomes across cell lines or had no impact on cell migration and drug response $(8,9)$. Thus, it appears crucial that a therapeutically useful RSK4 inhibitor should avoid targeting RSK1 at the least.

Prior crystal structure data for the NTKDs of RSK1 and 2 (18) compared to that shown for RSK4 in the present paper reveal that the active sites for all three kinases are essentially identical. Consequently, the best hope of a RSK4 selective inhibitor might be a molecule that binds allosterically rather than 
in an ATP competitive fashion. The floxacins were originally developed as antibiotics (42) but our results show that some members of this family appear to selectively inhibit the activation of RSK4 through allosteric binding. This occurs via a second AMP-PNP binding site not conservered in other AGC kinases providing a likely mechanism for RSK4-selectivity. Several questions arise from our observations including whether the antibiotic function of floxacins is necessary for inhibition of RSK4 activation and whether floxacins could be re-purposed for use as an anticancer agent in the clinic?

A clue to answering the first question is provided by our finding that whilst all floxacins are antibtioics, several family members such as ciprofloxacin and danofloxacin fail to impair RSK4 activation. This combined with the information on the RSK4 binding site for active floxacins should enable chemistry to derive new allosteric inhibitors lacking the antibiotic function but nevertheless maintain RSK4 targeting. Loss of floxacin antibiotic function could be clinically advantageous in potential cancer therapy. This is because prolonged use of these agents can cause serious antibioticinduced complications including severe diarrohea due to clostridium difficile toxin as well as facilitating a rise in bacterial antibiotic resistance $(43,44)$. It is unknown whether dissociation of antibiotic from RSK4 targeting effects of floxacins will be possible and whether this would remove all unwanted side-effects, but it seems likely it would eliminate the diarrhoea and issues related to bacterial resistance. Intriguingly, results from several studies in cancer patients receiving chemotherapy provide tentative hope that at least one RSK4 targeting floxacin, levofloxacin, is well tolerated (44). Whilst there is good data to show that use of floxacins such as levofloxacin, prevents neutropaenic sepsis and it's related acute deaths in cancer patients undergoing chemotherapy, there was no evidence to inducate that this might improve longer-term survival. Data presented here suggest that at least for levofloxacin, cancer patients taking this drug as part of the SIGNIGICANT trial do survive longer leading us to speculate that this benefit could be due to inhibition of RSK4. However, other reports show that floxacins may exert distinct effects on cancer cells such as impaired mitochondrial function and oxidative stress responses (45) which may be independent of RSK4 targeting so further work is needed.

So how could RSK4 inihibtion impair cell migration/invasion and enhance chemotherapeutic effects in lung and bladder cancer cells? Prior data in other RSKs across several cancer types have not revealed any clear mechanistic data for RSK4 (46). Here, we have provided some evidence that RSK4 enhances resistance to chemotherapeutic drugs at least in part by increasing cIAP2 protein levels through a transcriptional mechanism. However, how enhanced transcription of its mRNA is achieved is not yet clear although this is not dependent on NFkB. We also provide some evidence that RSK4 phosphorlyates Ser89 of p300 likely leading to increased RELA nuclear localisation and enhanced activity of NFkB thereby increasing EMT and metastatic behaviour.

There are several limitations of our study and some are now discussed. Firstly, we have not provided a formal demonstration of trovafloxacin specificity for inhibiting RSK4 as opposed to RSK1 activation. This would require the development of an in vitro RSK1 activation assay which is complicated by the fact that both ERK2 and PDK1 would be needed and our attempt to develop this have so far failed. However, our preliminary data show that trovafloxacin does not inhibit the activation of RSK1 in cells. Secondly, further research is required to provide definitive proof of endogenous interaction between RSK4 and p300 and to conclusively show how this mediates the EMT changes observed. Thirdly, although our prior research (8) and results shown here demonstrate that RSK1 and RSK4 
have opposite biological outputs in regulating invasion and chemo-sensitivity of lung cancer cells, the downstream mediators responsible are currently unclear. Large scale omics analysis assessing the effects of RSK1 and RSK4 silencing and over-expression are required to identify potential discriminating effector pathways. Forthly, some, but not all floxacins, are potent RSK4 activation inhibitors. However, our current data do not provide sufficient structure-activity relationship information to clearly define the reason behind this selectivity. Finally, the re-analysed SIGNIFICANT trial data do not establish that the reason for improved survival with levofloxacin is anything to do with RSK4 inhibition. Indeed, a proof of concept trial is urgently needed to test this hypothesis in patients.

In summary, we have identified RSK4 as a mediator of tumour metastasis and chemotherapy resistance in vitro and in vivo and as a potential therapeutic target in lung and bladder cancer. Our drug screen identified certain floxacins as RSK4 allosteric inhibitors with our structural and in-silico modelling work providing a potential site for their binding. Collectively, our data suggest that RSK4targeting floxacins such as levofloxacin could be re-purposed for proof-of-concept combination studies with existing chemotherapeutics in patients with lung and bladder cancer.

\section{Materials and Methods}

Study design

The aim of this study was to highlight the role of RSK4 in invasiveness and chemoresistance of lung and bladder cancer cells and show how targeting this kinase may represent an effective therapeutic strategy for these cancers. We silenced or induced RSK4 expression in lung AdCa and bladder cancer cell lines and studied the molecular mechanisms involved in modulating the above processes using molecular biology, biochemical, and phenotypic analysis. We also screened for and identified RSK4 activation inhibitors that reproduced the molecular and phenotypic changes observed through RSK4 silencing. We validated our findings through varied corresponding mouse or explant models and proposed mechanism of action for the inhibitors based on structural, mathematical, and biochemical approaches. For in vitro or cell culture experiments, at least three independent biological replicates per condition were used for statistical analysis. For animal experiments, the work was performed under UK Home office approved project licenses and in accordance with institutional welfare guidelines. Analysis of $\mathrm{Ct}$ scan images to measure changes in tumour volume was done blinded. Animals were treated without knowledge of anticipated outcomes. No data points were removed as outliers.Cohort sizes were selected based on prior work using the same models and corresponding power analyses to provide power of 0.8 and $\mathrm{P}<0.05$. Tumour-bearing animals were randomized before treatment. Animals showing no tumour growth prior to treatment administration were excluded from further analysis. Last, we searched for prior clinical trials using floxacins in cancers including lung and bladder to ask if there was any evidence that use of such antibiotics might extend patient survival. Further methods details are provided in the Supplementary Materials.

\section{Statistical analysis}

Statistical testing was performed using Prism (GraphPad) or Excel (Microsoft). Most experiments were analyzed by one-way ANOVA with Tukey's, Dunnett's or Benjamini-Hochberg correction multiple comparison or with two-tailed unpaired Student's t-test. For datasets where the reduced count did not allow normality test, a normal distribution was assumed on the basis of data distribution. For all statistical analyses, significance was accepted at the $95 \%$ confidence level $(P<0.05)$ and significance 
levels indicated. Error bars in all figures represent the SEM as a measure of the accuracy of the mean values shown unless otherwise stated. 


\section{List of Supplementary Materials}

Materials and Methods

Fig S1: Kinome siRNA screen hits in T24 cells and validation results

Fig S2: RSK4 modulates chemoresistance in NSCLC cells

Fig S3: RSK4 modulates the metastatic program in lung and bladder cancer cells

Fig S4: Active RELA overexpression rescues cell migration but not drug response changes downstream of RSK4 silencing

Fig S5: RSK4 overexpression in lung cancer cells and clinical relevance.

Fig S6: RSK4 is overexpressed in bladder cancer

Fig S7: RSK4 downregulation prevents lung colonisation by A549 cells

Fig S8: Rational for and identification of a RSK4 allosteric inhibitor

Fig S9: Not all floxacins inhibit RSK4 activation

Fig S10: Floxacin compounds tested in our RSK4 activation assay, corresponding structures, and pIC50 values

Fig S11: Floxacins inhibiting RSK4 reproduce cellular effects of RSK4 knockdown

Fig S12: RSK4 structures and floxacin docking

Fig S13: Identifying mutations that perturb the communication between the active site and allosteric site through exhaustive computational alanisation

Fig S14: Deuterium exchange mass-spectrometry

Fig S15: The hydrophobic motif of RSK4 modulates the activity of the RSK4-NTKD

Fig S16: Trovafloxacin potentiates the effects of cisplatin treatment on tumours in vivo in mice and ex vivo on patient tumour explants

Fig S17: CONSORT flow diagram of the SIGNIFICANT Trial

Fig S18: Levofloxacin added to cisplatin-based chemotherapy improves survival in patients with cancers including lung and bladder

Table S1: Summary of data collection, structure determination, and refinement statistics for the RSK4-NTKD, RSK4-NTKD pS232 and RSK4-NTKD S232E crystals.

Table S2: siRNA sequences used to target RSK4

Table S3: RT-qPCR primers used

Data file S1. Trovafloxacin has no major activity against a panel of 140 recombinant kinases.

(Excel)Data file S2. Raw data.

Movie S1. Micro-CT projection of lung of an exemplar vehicle-only treated KP mouse

Movie S2. Micro-CT projection of lung of an exemplar trovafloxacin/cisplatin combination treated KP mouse

\section{References and notes}

1. R. Lara, M. J. Seckl, O. E. Pardo, The p90 RSK family members: common functions and isoform specificity. Cancer Res 73, 5301-5308 (2013).

2. Y. Romeo, P. P. Roux, Paving the way for targeting RSK in cancer. Expert Opin Ther Targets 15, 5-9 (2011). 
3. A. Thakur, Y. Sun, A. Bollig, J. Wu, H. Biliran, S. Banerjee, F. H. Sarkar, D. J. Liao, Anti-invasive and antimetastatic activities of ribosomal protein $\mathrm{S} 6$ kinase 4 in breast cancer cells. Clin Cancer Res 14, 4427-4436 (2008).

4. L. Lopez-Vicente, G. Armengol, B. Pons, L. Coch, E. Argelaguet, M. Lleonart, J. HernandezLosa, I. de Torres, S. Ramon y Cajal, Regulation of replicative and stress-induced senescence by RSK4, which is down-regulated in human tumors. Clin Cancer Res 15, 4546-4553 (2009).

5. K. Berns, E. M. Hijmans, J. Mullenders, T. R. Brummelkamp, A. Velds, M. Heimerikx, R. M. Kerkhoven, M. Madiredjo, W. Nijkamp, B. Weigelt, R. Agami, W. Ge, G. Cavet, P. S. Linsley, R. L. Beijersbergen, R. Bernards, A large-scale RNAi screen in human cells identifies new components of the p53 pathway. Nature 428, 431-437 (2004).

6. C. Bender, A. Ullrich, PRKX, TTBK2 and RSK4 expression causes Sunitinib resistance in kidney carcinoma- and melanoma-cell lines. International journal of cancer 131, E45-55 (2012).

7. L. Fan, P. Li, Z. Yin, G. Fu, D. J. Liao, Y. Liu, J. Zhu, Y. Zhang, L. Wang, Q. Yan, Y. Guo, C. Shao, G. Huang, Z. Wang, Ribosomal s6 protein kinase 4: a prognostic factor for renal cell carcinoma. Br J Cancer 109, 1137-1146 (2013).

8. R. Lara, F. A. Mauri, H. Taylor, R. Derua, A. Shia, C. Gray, A. Nicols, R. J. Shiner, E. Schofield, P. A. Bates, E. Waelkens, M. Dallman, J. Lamb, D. Zicha, J. Downward, M. J. Seckl, O. E. Pardo, An siRNA screen identifies RSK1 as a key modulator of lung cancer metastasis. Oncogene, (2011).

9. C. Swanton, M. Marani, O. Pardo, P. H. Warne, G. Kelly, E. Sahai, F. Elustondo, J. Chang, J. Temple, A. A. Ahmed, J. D. Brenton, J. Downward, B. Nicke, Regulators of mitotic arrest and ceramide metabolism are determinants of sensitivity to paclitaxel and other chemotherapeutic drugs. Cancer cell 11, 498-512 (2007).

10. O. E. Pardo, A. Lesay, A. Arcaro, R. Lopes, B. L. Ng, P. H. Warne, I. A. McNeish, T. D. Tetley, N. R. Lemoine, H. Mehmet, M. J. Seckl, J. Downward, Fibroblast growth factor 2-mediated translational control of IAPs blocks mitochondrial release of Smac/DIABLO and apoptosis in small cell lung cancer cells. Mol Cell Biol 23, 7600-7610 (2003).

11. B. Bonavida, S. Baritaki, The novel role of Yin Yang 1 in the regulation of epithelial to mesenchymal transition in cancer via the dysregulated NF-kappaB/Snail/YY1/RKIP/PTEN Circuitry. Crit Rev Oncog 16, 211-226 (2011).

12. R. Kiernan, V. Bres, R. W. Ng, M. P. Coudart, S. El Messaoudi, C. Sardet, D. Y. Jin, S. Emiliani, M. Benkirane, Post-activation turn-off of NF-kappa B-dependent transcription is regulated by acetylation of p65. J Biol Chem 278, 2758-2766 (2003).

13. L. W. Yuan, J. W. Soh, I. B. Weinstein, Inhibition of histone acetyltransferase function of p300 by PKCdelta. Biochimica et biophysica acta 1592, 205-211 (2002).

14. C. D. Simpson, K. Anyiwe, A. D. Schimmer, Anoikis resistance and tumor metastasis. Cancer Lett 272, 177-185 (2008).

15. M. K. Hilinski, R. M. Mrozowski, D. E. Clark, D. A. Lannigan, Analogs of the RSK inhibitor SL0101: optimization of in vitro biological stability. Bioorg Med Chem Lett 22, 3244-3247 (2012).

16. G. P. Sapkota, L. Cummings, F. S. Newell, C. Armstrong, J. Bain, M. Frodin, M. Grauert, M. Hoffmann, G. Schnapp, M. Steegmaier, P. Cohen, D. R. Alessi, BI-D1870 is a specific inhibitor of the p90 RSK (ribosomal S6 kinase) isoforms in vitro and in vivo. Biochem J 401, 29-38 (2007).

17. J. A. Endicott, M. E. M. Noble, L. N. Johnson, The Structural Basis for Control of Eukaryotic Protein Kinases. Annual Review of Biochemistry, Vol 81 81, 587-613 (2012).

18. M. Malakhova, I. Kurinov, K. Liu, D. Zheng, I. D'Angelo, J. H. Shim, V. Steinman, A. M. Bode, Z. Dong, Structural diversity of the active $\mathrm{N}$-terminal kinase domain of p90 ribosomal S6 kinase 2. PLoS One 4, e8044 (2009). 
19. K. J. Smith, P. S. Carter, A. Bridges, P. Horrocks, C. Lewis, G. Pettman, A. Clarke, M. Brown, J. Hughes, M. Wilkinson, B. Bax, A. Reith, The structure of MSK1 reveals a novel autoinhibitory conformation for a dual kinase protein. Structure 12, 1067-1077 (2004).

20. B. A. Dummler, C. Hauge, J. Silber, H. G. Yntema, L. S. Kruse, B. Kofoed, B. A. Hemmings, D. R. Alessi, M. Frodin, Functional characterization of human RSK4, a new 90-kDa ribosomal S6 kinase, reveals constitutive activation in most cell types. Journal of Biological Chemistry 280, 13304-13314 (2005).

21. A. Krantz, Probing protein surfaces for 'hot spots': a new frontier. Trends Biotechnol 16, 198199 (1998).

22. L. R. Pearce, D. Komander, D. R. Alessi, The nuts and bolts of AGC protein kinases. Nature reviews 11, 9-22 (2010).

23. J. Yang, P. Cron, V. Thompson, V. M. Good, D. Hess, B. A. Hemmings, D. Barford, Molecular mechanism for the regulation of protein kinase B/Akt by hydrophobic motif phosphorylation. Molecular cell 9, 1227-1240 (2002).

24. M. Frodin, T. L. Antal, B. A. Dummler, C. J. Jensen, M. Deak, S. Gammeltoft, R. M. Biondi, A phosphoserine/threonine-binding pocket in AGC kinases and PDK1 mediates activation by hydrophobic motif phosphorylation. Embo Journal 21, 5396-5407 (2002).

25. S. J. De Vries, A. D. J. van Dijk, M. Krzeminski, M. van Dijk, A. Thureau, V. Hsu, T. Wassenaar, A. M. J. J. Bonvin, HADDOCK versus HADDOCK: New features and performance of HADDOCK2.0 on the CAPRI targets. Proteins 69, 726-733 (2007).

26. G. C. P. van Zundert, J. P. G. L. M. Rodrigues, M. Trellet, C. Schmitz, P. L. Kastritis, E. Karaca, A. S. J. Melquiond, M. van Dijk, S. J. de Vries, A. M. J. J. Bonvin, The HADDOCK2.2 Web Server: User-Friendly Integrative Modeling of Biomolecular Complexes. Journal of Molecular Biology 428, 720-725 (2016).

27. B. Amor, S. N. Yaliraki, R. Woscholski, M. Barahona, Uncovering allosteric pathways in caspase-1 using Markov transient analysis and multiscale community detection. Mol Biosyst 10, 2247-2258 (2014).

28. R. Koenker, Quantile Regression. (2005).

29. R. Koenker, Quantreg: Quantile Regression. R package version 5.19. Available at http://CRAN.R-project.org/package=quantreg., ( 2015).

30. O. Trott, A. J. Olson, Software News and Update AutoDock Vina: Improving the Speed and Accuracy of Docking with a New Scoring Function, Efficient Optimization, and Multithreading. Journal of Computational Chemistry 31, 455-461 (2010).

31. R. A. Friesner, R. B. Murphy, M. P. Repasky, L. L. Frye, J. R. Greenwood, T. A. Halgren, P. C. Sanschagrin, D. T. Mainz, Extra precision glide: docking and scoring incorporating a model of hydrophobic enclosure for protein-ligand complexes. J Med Chem 49, 6177-6196 (2006).

32. T. E. Wales, J. R. Engen, Hydrogen exchange mass spectrometry for the analysis of protein dynamics. Mass Spectrom Rev 25, 158-170 (2006).

33. O. Vadas, H. A. Dbouk, A. Shymanets, O. Perisic, J. E. Burke, W. F. Abi Saab, B. D. Khalil, C. Harteneck, A. R. Bresnick, B. Nurnberg, J. M. Backer, R. L. Williams, Molecular determinants of PI3Kgamma-mediated activation downstream of G-protein-coupled receptors (GPCRs). Proc Natl Acad Sci U S A 110, 18862-18867 (2013).

34. K. Y. Chung, S. G. Rasmussen, T. Liu, S. Li, B. T. DeVree, P. S. Chae, D. Calinski, B. K. Kobilka, V. L. Woods, Jr., R. K. Sunahara, Conformational changes in the $G$ protein Gs induced by the beta2 adrenergic receptor. Nature 477, 611-615 (2011).

35. M. DuPage, A. L. Dooley, T. Jacks, Conditional mouse lung cancer models using adenoviral or lentiviral delivery of Cre recombinase. Nat Protoc 4, 1064-1072 (2009).

36. R. Ben-Hamo, A. Jacob Berger, N. Gavert, M. Miller, G. Pines, R. Oren, E. Pikarsky, C. H. Benes, T. Neuman, Y. Zwang, S. Efroni, G. Getz, R. Straussman, Predicting and affecting response to cancer therapy based on pathway-level biomarkers. Nature communications 11, 3296 (2020). 
37. M. Cullen, N. Steven, L. Billingham, C. Gaunt, M. Hastings, P. Simmonds, N. Stuart, D. Rea, M. Bower, I. Fernando, R. Huddart, S. Gollins, A. Stanley, Antibacterial prophylaxis after chemotherapy for solid tumors and lymphomas. N Engl J Med 353, 988-998 (2005).

38. G. Cao, Y. Zhu, X. Xie, Y. Chen, J. Yu, J. Zhang, Z. Chen, L. Pang, Y. Zhang, Y. Shi, Pharmacokinetics and pharmacodynamics of levofloxacin in bronchial mucosa and lung tissue of patients undergoing pulmonary operation. Exp Ther Med 20, 607-616 (2020).

39. D. N. Fish, A. T. Chow, The clinical pharmacokinetics of levofloxacin. Clin Pharmacokinet 32, 101-119 (1997).

40. J. Zhu, Q. Y. Li, J. L. Liu, W. Wei, H. W. Yang, W. Tang, RSK4 knockdown promotes proliferation, migration and metastasis of human breast adenocarcinoma cells. Oncol Rep 34, 3156-3162 (2015).

41. S. C. Samson, A. Elliott, B. D. Mueller, Y. Kim, K. R. Carney, J. P. Bergman, J. Blenis, M. C. Mendoza, p90 ribosomal S6 kinase (RSK) phosphorylates myosin phosphatase and thereby controls edge dynamics during cell migration. J Biol Chem 294, 10846-10862 (2019).

42. K. J. Aldred, R. J. Kerns, N. Osheroff, Mechanism of quinolone action and resistance. Biochemistry 53, 1565-1574 (2014).

43. L. S. M. Kuula, K. M. Viljemaa, J. T. Backman, M. Blom, Fluoroquinolone-related adverse events resulting in health service use and costs: A systematic review. PLoS One 14, e0216029 (2019).

44. S. S. F. Lee, A. E. Fulford, M. A. Quinn, J. Seabrook, I. Rajakumar, Levofloxacin for febrile neutropenia prophylaxis in acute myeloid leukemia patients associated with reduction in hospital admissions. Support Care Cancer 26, 1499-1504 (2018).

45. V. Yadav, P. Talwar, Repositioning of fluoroquinolones from antibiotic to anti-cancer agents: An underestimated truth. Biomed Pharmacother 111, 934-946 (2019).

46. R. Cronin, G. N. Brooke, F. Prischi, The role of the p90 ribosomal S6 kinase family in prostate cancer progression and therapy resistance. Oncogene, (2021).

48. C. Swanton, M. Marani, O. Pardo, P. H. Warne, G. Kelly, E. Sahai, F. Elustondo, J. Chang, J. Temple, A. A. Ahmed, J. D. Brenton, J. Downward, B. Nicke, Regulators of mitotic arrest and ceramide metabolism are determinants of sensitivity to paclitaxel and other chemotherapeutic drugs. Cancer cell 11, 498-512 (2007).

49. L. F. Chen, S. A. Williams, Y. Mu, H. Nakano, J. M. Duerr, L. Buckbinder, W. C. Greene, NFkappaB RelA phosphorylation regulates RelA acetylation. Mol Cell Biol 25, 7966-7975 (2005).

50. X. Li, Y. Yang, J. D. Ashwell, TNF-RII and C-IAP1 mediate ubiquitination and degradation of TRAF2. Nature 416, 345-347 (2002).

51. B. Herberger, H. Puhalla, M. Lehnert, F. Wrba, S. Novak, A. Brandstetter, B. Gruenberger, T. Gruenberger, R. Pirker, M. Filipits, Activated mammalian target of rapamycin is an adverse prognostic factor in patients with biliary tract adenocarcinoma. Clin Cancer Res 13, 47954799 (2007).

52. J. Bain, H. McLauchlan, M. Elliott, P. Cohen, The specificities of protein kinase inhibitors: an update. Biochem J 371, 199-204 (2003).

53. F. A. Ran, P. D. Hsu, J. Wright, V. Agarwala, D. A. Scott, F. Zhang, Genome engineering using the CRISPR-Cas9 system. Nat Protoc 8, 2281-2308 (2013).

54. J. S. Lee, S. H. Leem, S. Y. Lee, S. C. Kim, E. S. Park, S. B. Kim, S. K. Kim, Y. J. Kim, W. J. Kim, I. S. Chu, Expression signature of E2F1 and its associated genes predict superficial to invasive progression of bladder tumors. J Clin Oncol 28, 2660-2667 (2010).

55. M. Sanchez-Carbayo, N. D. Socci, J. Lozano, F. Saint, C. Cordon-Cardo, Defining molecular profiles of poor outcome in patients with invasive bladder cancer using oligonucleotide microarrays. J Clin Oncol 24, 778-789 (2006).

56. M. Malakhova, I. Kurinov, K. Liu, D. Zheng, I. D'Angelo, J. H. Shim, V. Steinman, A. M. Bode, Z. Dong, Structural diversity of the active $\mathrm{N}$-terminal kinase domain of p90 ribosomal S6 kinase 2. PLoS One 4, e8044 (2009). 
57. W. Kabsch, XDS. Acta crystallographica. Section D, Biological crystallography 66, 125-132 (2010).

58. A. J. Mccoy, R. W. Grosse-Kunstleve, P. D. Adams, M. D. Winn, L. C. Storoni, R. J. Read, Phaser crystallographic software. J Appl Crystallogr 40, 658-674 (2007).

59. N. S. Pannu, G. N. Murshudov, E. J. Dodson, R. J. Read, Incorporation of prior phase information strengthens maximum-likelihood structure refinement. Acta Crystallogr D 54, 1285-1294 (1998).

60. P. D. Adams, P. V. Afonine, G. Bunkoczi, V. B. Chen, I. W. Davis, N. Echols, J. J. Headd, L. W. Hung, G. J. Kapral, R. W. Grosse-Kunstleve, A. J. McCoy, N. W. Moriarty, R. Oeffner, R. J. Read, D. C. Richardson, J. S. Richardson, T. C. Terwilliger, P. H. Zwart, PHENIX: a comprehensive Python-based system for macromolecular structure solution. Acta Crystallogr D 66, 213-221 (2010).

61. P. Emsley, B. Lohkamp, W. G. Scott, K. Cowtan, Features and development of Coot. Acta Crystallogr D 66, 486-501 (2010).

62. S. J. De Vries, A. D. J. van Dijk, M. Krzeminski, M. van Dijk, A. Thureau, V. Hsu, T. Wassenaar, A. M. J. J. Bonvin, HADDOCK versus HADDOCK: New features and performance of HADDOCK2.0 on the CAPRI targets. Proteins 69, 726-733 (2007).

63. O. Trott, A. J. Olson, Software News and Update AutoDock Vina: Improving the Speed and Accuracy of Docking with a New Scoring Function, Efficient Optimization, and Multithreading. Journal of Computational Chemistry 31, 455-461 (2010).

64. C. Meier, D. C. Brookings, T. A. Ceska, C. Doyle, H. P. Gong, D. McMillan, G. P. Saville, A. Mushtaq, D. Knight, S. Reich, L. H. Pearl, K. A. Powell, R. Sawa, R. A. Allen, Engineering human MEK-1 for structural studies: A case study of combinatorial domain hunting. Journal of Structural Biology 177, 329-334 (2012).

65. W. P. Feinstein, M. Brylinski, Calculating an optimal box size for ligand docking and virtual screening against experimental and predicted binding pockets. J Cheminformatics 7, (2015).

66. R. A. Friesner, R. B. Murphy, M. P. Repasky, L. L. Frye, J. R. Greenwood, T. A. Halgren, P. C. Sanschagrin, D. T. Mainz, Extra precision glide: docking and scoring incorporating a model of hydrophobic enclosure for protein-ligand complexes. J Med Chem 49, 6177-6196 (2006).

67. M. P. Jacobson, D. L. Pincus, C. S. Rapp, T. J. Day, B. Honig, D. E. Shaw, R. A. Friesner, A hierarchical approach to all-atom protein loop prediction. Proteins 55, 351-367 (2004).

68. L. Whitmore, B. A. Wallace, Protein secondary structure analyses from circular dichroism spectroscopy: methods and reference databases. Biopolymers 89, 392-400 (2008).

69. L. Whitmore, B. A. Wallace, DICHROWEB, an online server for protein secondary structure analyses from circular dichroism spectroscopic data. Nucleic Acids Res 32, W668-673 (2004).

70. J. C. Silva, R. Denny, C. A. Dorschel, M. Gorenstein, I. J. Kass, G. Z. Li, T. McKenna, M. J. Nold, K. Richardson, P. Young, S. Geromanos, Quantitative proteomic analysis by accurate mass retention time pairs. Anal Chem 77, 2187-2200 (2005).

71. R. Ben-Hamo, A. Jacob Berger, N. Gavert, M. Miller, G. Pines, R. Oren, E. Pikarsky, C. H. Benes, T. Neuman, Y. Zwang, S. Efroni, G. Getz, R. Straussman, Predicting and affecting response to cancer therapy based on pathway-level biomarkers. Nature communications 11 , 3296 (2020).

72. T. Valovka, F. Verdier, R. Cramer, A. Zhyvoloup, T. Fenton, H. Rebholz, M. L. Wang, M. Gzhegotsky, A. Lutsyk, G. Matsuka, V. Filonenko, L. Wang, C. G. Proud, P. J. Parker, I. T. Gout, Protein kinase $\mathrm{C}$ phosphorylates ribosomal protein $\mathrm{S} 6$ kinase betall and regulates its subcellular localization. Mol Cell Biol 23, 852-863 (2003).

73. Y. Romeo, X. Zhang, P. P. Roux, Regulation and function of the RSK family of protein kinases. Biochem J 441, 553-569 (2012).

74. J. A. Endicott, M. E. M. Noble, L. N. Johnson, The Structural Basis for Control of Eukaryotic Protein Kinases. Annual Review of Biochemistry, Vol 81 81, 587-613 (2012). 
75. A. Lanczky, A. Nagy, G. Bottai, G. Munkacsy, A. Szabo, L. Santarpia, B. Gyorffy, miRpower: a web-tool to validate survival-associated miRNAs utilizing expression data from 2178 breast cancer patients. Breast Cancer Res Treat 160, 439-446 (2016).

\section{Acknowledgements}

The authors gratefully acknowledge infrastructure support from the Cancer Research UK Imperial Centre, the Imperial Experimental Cancer Medicine Centre and the National Institute for Health Research Imperial Biomedical Research Centre. OEP, MJS, FP thank Cancer Research UK (CRUK) for funding SC, UM and FP, and CTRT for funding RR. MMUA acknowledges CRUK funding (C33269/A11161 and C33269/A20752). SNY and MB acknowledge Engineering and Physical Sciences Research Council (EPSRC) grant EP/N014529/1 supporting the EPSRC Centre for Mathematics of Precision Healthcare. DJP thank the Wellcome Trust Strategic Fund (PS3416). MMUA thanks CDF CRUK funding (A11161). JD is funded by the Francis Crick Institute-which receives its core funding from Cancer Research UK (FC001070), the UK Medical Research Council (FC001070), and the Wellcome Trust (FC001070).

Competing Interests: OEP is scientific adviser for ClyzLabs Limited. JD acted as consultant for AstraZeneca, Bayer, Jubilant, Theras, Vividion and Novartis, and has funded research agreements with BMS, Revolution Medicines and Boehringer Ingelheim. PC is on the Scientific Advisory Board of Mission Therapeutics. SJS, VB and AZ are employed by Curesponse. DJP received lecture fees from ViiV Healthcare, Bayer Healthcare, Falk, BMS, EISAI and Roche, travel expenses from BMS, MSD and Bayer Healthcare, consulting fees for Mina Therapeutics, EISAI, H3B, Roche, Astra Zeneca and DaVolterra. RL is employed by AstraZeneca and a Biosceptre shareholder. $\mathrm{DM} / \mathrm{LC} / \mathrm{JP} / \mathrm{MW} / \mathrm{CM} / \mathrm{FM} / \mathrm{CG} / \mathrm{JMS} / \mathrm{SO} / \mathrm{MM}-\mathrm{A} / \mathrm{DH} / \mathrm{MC} / \mathrm{UM} / \mathrm{MMUA} / \mathrm{FP} / \mathrm{SP} / \mathrm{YW} / \mathrm{LT} / \mathrm{MB} / \mathrm{SG} / \mathrm{GG}$ declare that they have no competing interests.

Data and materials availability: Coordinates for the RSK4 NTKD structures are deposited with the Protein Data Bank: RSK4 NTKD (6G77), RSK4 NTKD pS232 (6G76) and RSK4 NTKD S232E (6G78). Some plasmids used in this study are covered by MTA: RELA, MTA\#2020S-1003-1024, Addgene; cIAP1 and 2, MTA\#2018S-0908-1058, Addgene.

\section{Figure legends}

Figure 1: RSK4 downregulation sensitises lung and bladder cancer cell lines to chemotherapy. (A-C) A kinome siRNA screen reveals modulators of taxol and cisplatin response in T24 bladder cancer cells. (A) Dot plot of ratio of cell number change in response to drug treatment for cells silenced for individual targets over those transfected with non-targeting (NT) sequences. (B) Venn diagram of targets modulating response to cisplatin with or without taxol. (C) Number of validated targets in T24, TCCSUP, and J82 bladder cancer cells out of the 23 screen hits. (D) Effect of siRNA-mediated 
silencing in T24 or A549 or (E) CRISPR-mediated knock-out of RSK4 in A549 cells on cell survival to cisplatin (T24-3.5 $\mu \mathrm{M}$ and A549-12.5 $\mu \mathrm{M}$ ) or taxol (T24-40 nM and A549-58 nM). (E-upper) Lysates from RSK4 CRISPR clones (\#1,\#2) analysed by Western blotting (WB) for RSK4 knock-out efficiency. (E) \#1 and \#2 refer to two separate clones of RSK4-CRISPR cells. (F) RSK4 silencing effect on druginduced caspase-3/7 activation and (G) DNA damage in T24 and A549 cells. Cells transfected with RSK4-siRNA for 48h were treated with cisplatin or taxol and substrate-based caspase activity or $\gamma \mathrm{H} 2 \mathrm{AX}$ expression measured. (H) RSK4 silencing effect on baseline apoptotic pathway activity in A549 cells. (I) RSK4 downregulation effect on BCL2, cIAP1, and cIAP2 expression. (J) A549 cells treated with siRNA for $48 \mathrm{~h}$ analysed by RT-qPCR. (A, D and E) Cell number assessed by Crystal violet staining and normalised to the corresponding control condition. (A) Data are average of four individual siRNA sequences per target. (D-J) Data representative of $n=3$. (D-G and J) Data are mean \pm SEM of $n=4$. (D, H, I) Tubulin or vinculin was used as loading control.

Figure 2: RSK4 downregulation inhibits the pro-metastatic program. (A-G) A549 and T24 cells transfected with/without RSK4, RSK1 and non-targeting (NT) siRNAs or RSK4 and empty-vector (EV) cDNAs were analysed $48 \mathrm{~h}$ or $72 \mathrm{~h}$ later. (A) Random-walk assays determined cell speed (left) and single-cell trajectories (right). (B) Cells were covered with collagen-I and overlaid with EGF for chemotaxis. 48h later, cells were stained with Cytox Green before confocal imaging to determine the extent of invasion. Data are mean \pm SEM of 36 fields-of-view/condition. (C) Cells were stained for actin before fluorescent microscopy. Images are representative of 36 fields-of-view/condition. Scale bar: 10 $\mathrm{mm}$. (D) Cell lysates were analysed by SDS-PAGE/WB. Vinculin was used as loading controls. (E) Cells transfected with a RelA-Firefly luciferase reporter construct and a Renilla luciferase control vector were then transfected with siRNAs and lysates and analysed for Firefly luciferase activity normalised to the Renilla luciferase control. (F) cytoplasmic (Cyto) and nuclear (Nuc) fractions were analysed by SDS-PAGE/WB. Ubulin and Lamin B were used as loading controls. (G) Cell lysates were analysed by SDS-PAGE/WB. HSP90 was used as a loading control. (H) Recombinant p300 (1 $\mathrm{gg})$ was mixed with or without recombinant RSK4 (200ng) and $\gamma$ ATP for 5 min prior to SDS-PAGE/WB analysis. (I) A549 and T24 cells transfected with/without RSK4, RSK1 and non-targeting (NT) siRNAs were split equally $48 \mathrm{~h}$ later between adherent and ultra-low adhesion plates for $24 \mathrm{~h}$ prior to re-adhesion. Cell numbers were determined by Crystal violet staining and ratio of suspension-treated to onlyadherent cultures represented as mean $\pm S E M$ of $n=4$. Data are representative of at least biological triplicates. Statistics: Student's t-test (B, I and E) and ANOVA (A) with n.s.; not significant, *; $p<0.05$, $* * ; p<0.01, * * * ; p<0.001$.

Figure 3: RSK4 is overexpressed in lung cancer and correlates with poor survival in patients with lung adenocarcinoma. $(A-C)$ Normal $(n=25)$ and cancerous $(n=183)$ lung FFPE samples were stained with H\&E or a RSK4 antibody followed by 3,3'-Diaminobenzidinedevelopment. (A-left) Examples of RSK4 staining in normal lung and RSK4-negative and -positive adenocarcinoma (AdCa) samples. Scale bar: $30 \mu \mathrm{m}$. (A-right and B) Percentage of samples showing positive (pos) or negative (neg) RSK4 staining shown for (A) all primary (I ${ }^{\text {ary }}$ ) lung cancer samples or (B) lung cancer (LC) subtypes. SCC; squamous cell carcinoma, SCLC; small-cell LC. (C) RSK4 staining semi-quantitative scores for each LC subtype. (D) Comparison of RSK4 mRNA expression between AdCa, SCC, and normal lung samples in TCGA RNA-Seq data. Statistics: t-test; ns, not significant. (E) Overall survival of patients with AdCa or SCC with high or low tumour RSK4 mRNA expression. Cut-off: median value. (F) Comparison of post- 
mortem matched primary and metastatic AdCa $(n=26)$ and SCC $(n=20)$ lesions for RSK4 IHC staining scores. Percentage of cases where metastatic lesions expressed more (Met>Primary) or less (Met $\leq$ Primary) RSK4 than the primary site. Average IHC scores are shown. Statistics: (C) ANOVA; ***; $p<0.001, * * ; p<0.005$ and $*$; $p<0.05$.

Figure 4: RSK4 downregulation impairs growth of lung adenocarinoma xenografts, sensitises to chemotherapy, and reduces metastatic potential of lung cancer cells. (A-B) Non-targeting (NT) or RSK4 CRISPR A549 cells were injected subcutaneously in nude mice and tumour growth rate (A) or fold volume change at Day-46 following treatment cycle with/without cisplatin (B) monitored. (C) A459 NT or RSK4 CRISPR cell clone \#1 were injected through the tail vein of SCID mice. Animals were sacrificed 2 months later and their lungs paraffin-embedded. 10 sections (each separated by $60 \mu \mathrm{m}$ ) were $H \& E$ stained and numbers of (lower), and lung area colonised by (upper) tumour nodules quantified. Upper: dots represent individual lung sections. Statistics: ANOVA (A-B) and Student's ttest (C). n.s.; not significant, ${ }^{*} ; \mathrm{p}<0.05,{ }^{* *} ; \mathrm{p}<0.01,{ }^{* * *} ; \mathrm{p}<0.001$.

Figure 5: Trovafloxacin, an inhibitor of RSK4 activation, reproduces the biological effects of RSK4 silencing. (A) Diagram of the drug screening process. (B) Moxifloxacin, trovafloxacinand ciprofloxacin differ in their ability to inhibit activation of RSK4 by ERK2 in HTRF assays. (C-D) A549 cells incubated with the indicated concentration of floxacin for $1 \mathrm{~h}$ were treated with cisplatin (12.5 $\mu \mathrm{M})$ for $72 \mathrm{~h}(\mathrm{C})$ or $24 \mathrm{~h}$ (D). (C) Cell viability determined using Alamar Blue. (D) Caspase activity determined using a substrate-based fluorigenic assay. (E) Relation of cisplatin sensitisation by trovafloxacin in A549 cells to RSK4 expression. NT; non-targeting siRNA, EV; empty-vector DNA, oeRSK4; overexpressed RSK4, si5'UTR-RSK4; 5'UTR RSK4 mRNA-directed siRNA. (F) Lysates from A549 cells treated with or swithout trovafloxacin (Trova) for $24 \mathrm{~h}$ were analysed by SDS-PAGE/WB. Vinculin used as loading control. (G) Random cell migration or (H) 3D collagen-I invasion in A549 cells treated with indicated inhibitors $(10 \mu \mathrm{M})$. Dano; danofloxacin, Tosu; tosufloxacin tosylate, Moxi; moxifloxacin. (I) Trovafloxacin binding tos inactive (dephosphorylated, APO) and not fully-active (ATP) RSK4-NTKD. Tm-shift assay performed over the indicated temperature range (left) and parameters recorded (right). (B-H) Results representative of at least three independent repeats. Data are mean (B-D and F) or median (G-H) of $\mathrm{n}=4 \pm$ SEM. Statistics: ANOVA. $*$; $p \leq 0.05, * * ; p \leq 0.01, * * * ; p \leq 0.001$.

Figure 6: RSK4-NTKD structure and trovafloxacin binding site prediction. (A) Structure of dephosphorylated RSK4-NTKD. (B) Overlay of dephosphorylated RSK4-NTKD and PKA structures showing the characteristic $\beta B$-sheet and $\alpha 2$-helix of RSK4-NTKD as opposed to the C-helix of classical AGC kinases. (C-E) Docking of second AMP-PNP (C), the phospho-HM peptide (D) and trovafloxacin (E) to the RSK4-NTKD. Inserts: view of interaction residues. Colours: $\beta B$-sheet; red, activation; teal, $\mathrm{H}$-bonds; yellow dotted lines. AMP-PNP, trovafloxacin and hydrophobic motif FXXF-pS-F residues coloured by element. Key binding site residues shown in ball-and-stick. (F-G) Identification of an allosteric hotspot in RSK4 through Markov transient analysis. (F) Propagation of a random walk on the protein graph, originating at the active site showingactive site and a hotspot where trovafloxacin and AMP-PNP bind. The evolution of the probability distribution (shown at $t=0,500,1000 \mathrm{sec}$; atoms coloured from white to red with increasing probability) is shown relative to the trovafloxacin binding site and the upper and lower lobes of RSK4. (G) Top: From the RSK4 structure and location of its active site, the pathway is extracted through statistical quantile regression of the Markov transient times of the random walk, $\mathrm{t} 1 / 2$, against distance. Low quantile scores indicate residues highly connected to the active site (relative to their distance). Bottom: Quantile score ( $p R$ ) of each residue 
mapped onto RSK4's surface (different views). Residues below the $2 \%$ quantile connect the active (black ellipse) and the allosteric (green ellipse) sites. $(\mathrm{H})$ The propagation of the random walk originating from AMP-PNP or trovafloxacin binding at the allosteric site exhibits different communication pathways. (I) Deuterium exchange mass-spectrometry for RSK4 incubated with or without trovafloxacin. Difference plot obtained by subtracting the incorporation of deuterium in the absence versus in the presence of trovafloxacin. Colours: Experimental error; light grey, other colours represent different $\mathrm{D}_{2} \mathrm{O}$ incubation times. Upper half of graph; more exposed regions, lower half; protected regions. RSK4 amino acid numbers are given for regions showing significant protection or exposure as indicated by being above or below the dotted red and blue lines $(98 \%$ confidence limit).

Figure 7: Trovafloxacin enhances cisplatin efficacy in A549 xenografts and KRASV12/TP53-/- (KP)driven genetic mouse models. (A-B) Nude mice injected subcutaneously with luciferase-expressing A549 cells treated with or without trovafloxacin and cisplatin once tumours reached $0.5 \mathrm{~cm}$ in any dimension. (A) Left: representative images from each condition showing luciferase signal at day 0 and 14. Right: Percent tumour volume change at Day 14. Red bar; median. Statistics: Student's t-test, ${ }^{*} ; p \leq 0.05,{ }^{* *} ; p \leq 0.01,{ }^{* * *} ; p \leq 0.001$. (B) IHC of end-point tumours for caspase-3 cleavage. (C) Differential effect of trovafloxacin and ciprofloxacin on RSK4 phosphorylation (pRSK4-S232), cIAP2 expression and cleaved (Clv) caspase-3 in a cancer cell line isolated from KP tumours (D) Representative images of lungs from treated KP animals. Green stars; examples of lung tumour nodules in the vehicle-only condition. (E) Representative $\mu \mathrm{CT}$ scans from vehicle-only and combination-treated KP animals before treatment and at end-point. Individually tractable tumours indicated by numbers and green circles. (F) Percent volume change in individually tractable tumours in KP mice determined by differential analysis of CT-scans before treatment and at end-point. Dots represent individual tumours. Red line: median \pm SEM. Statistics: ANOVA; ${ }^{*} ; p \leq 0.05, * * ; p \leq 0.01,{ }^{* * *}$; $p \leq 0.005, * * * * ; p \leq 0.001$ and ns; non-significant. 
Fig 1

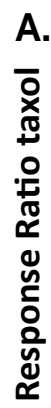

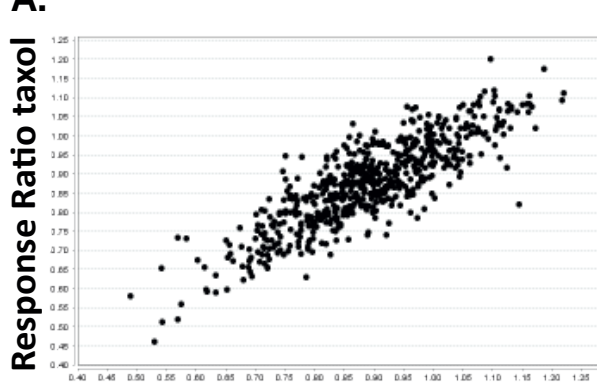

Response Ratio cisplatin

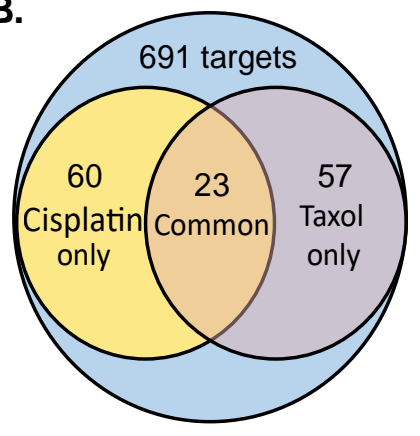

D.

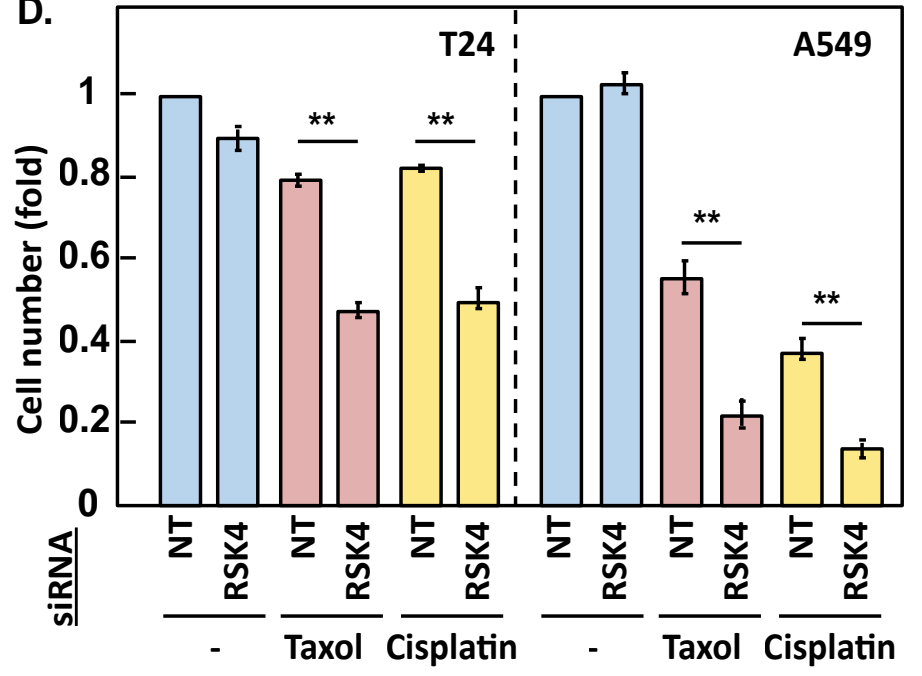

F.

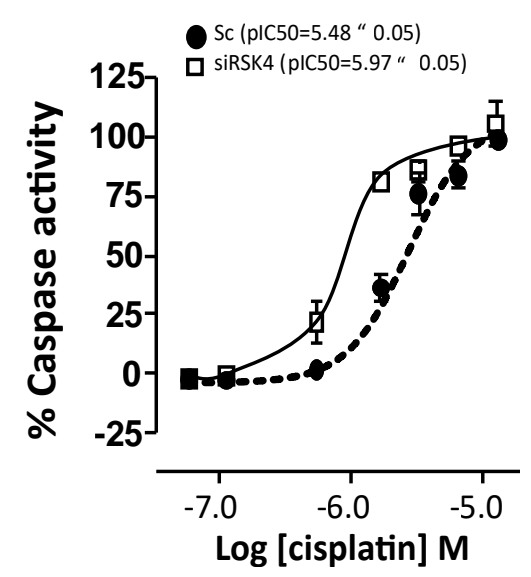

H.

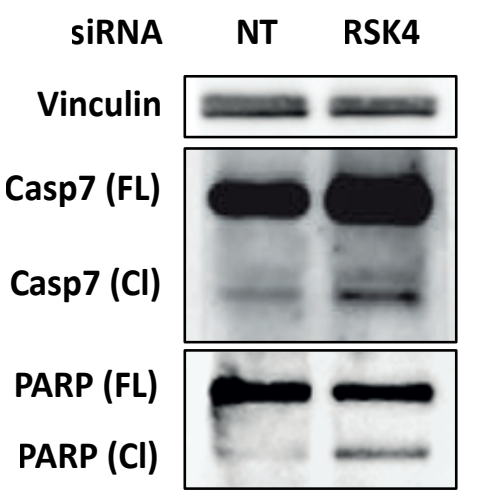

A549

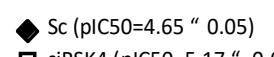

$\square$ siRSK4 (pIC50=5.17“ 0.05)
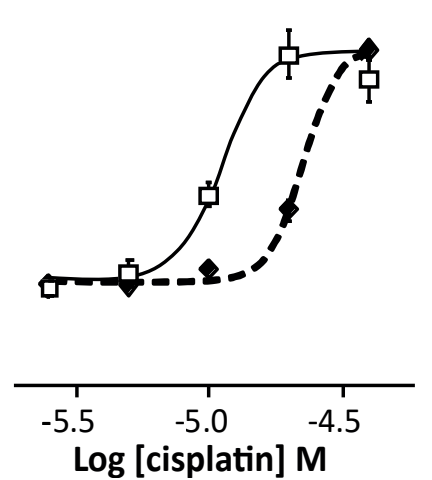

G. c.

\begin{tabular}{|l|c|c|c|c|}
\cline { 2 - 5 } \multicolumn{1}{c|}{} & T24 & T24 \& TCCSUP & T24 \& J82 & All 3 \\
\hline Cisplatin & 21 & 16 & 14 & 13 \\
\hline Taxol & 20 & 15 & 14 & 13 \\
\hline Both & 20 & 15 & 14 & 13 \\
\hline
\end{tabular}

E.

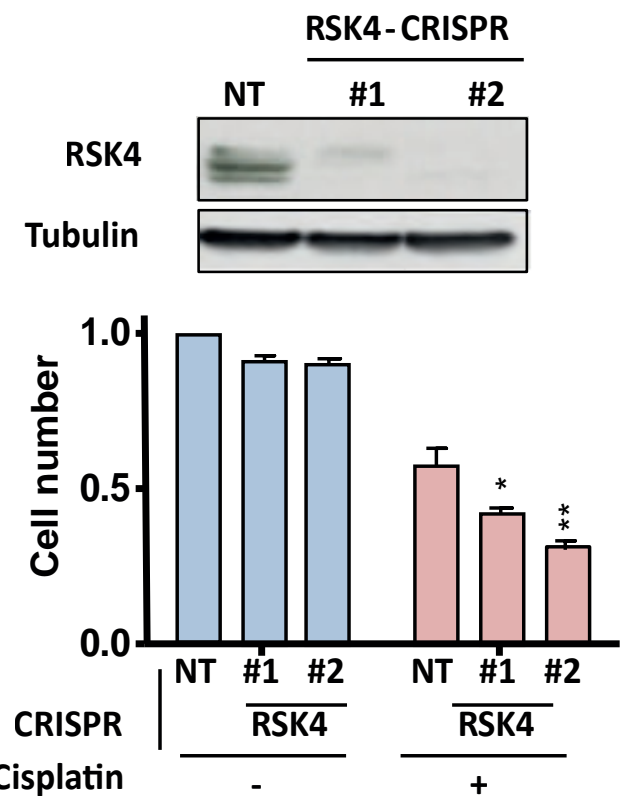

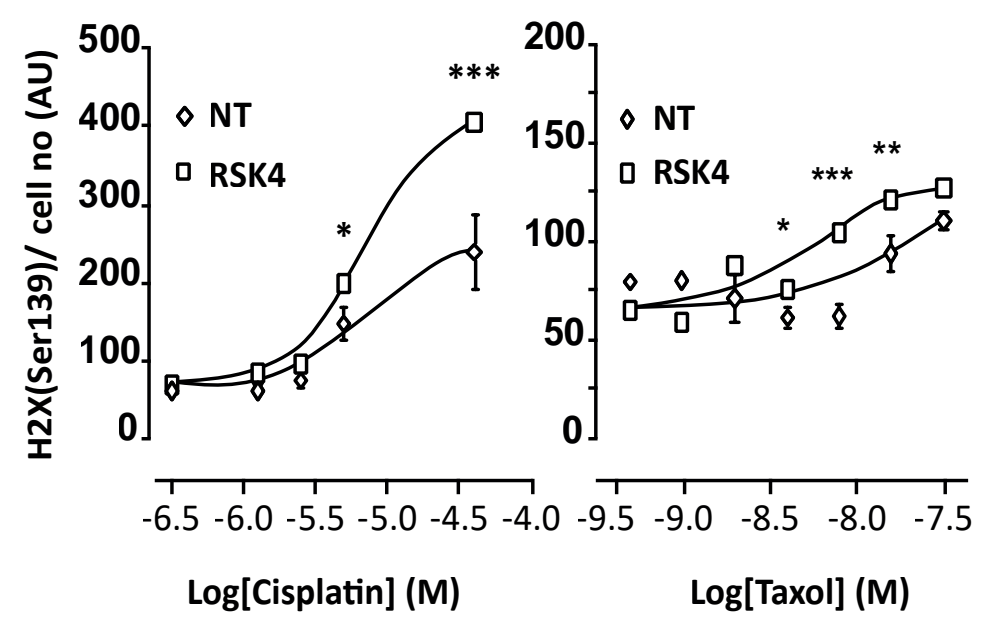

I.

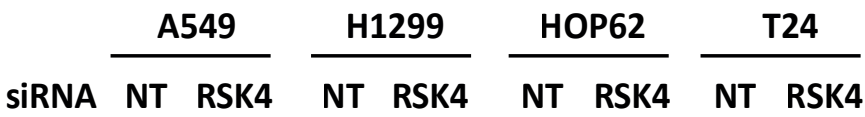

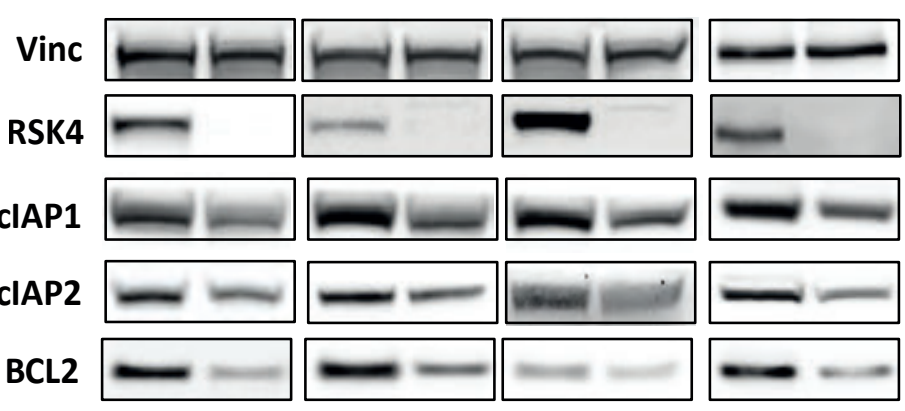

J.

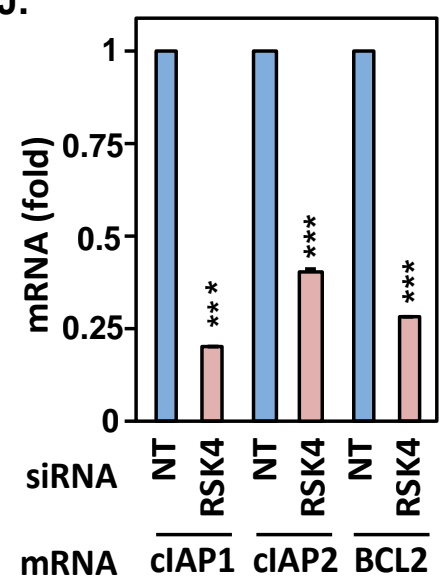


Fig 2

A.

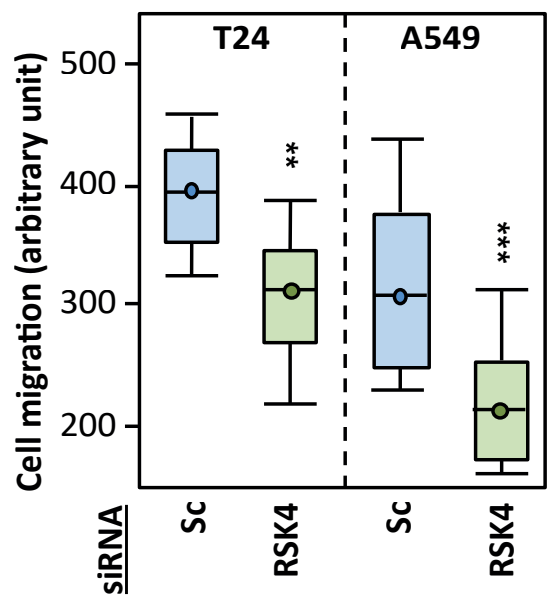

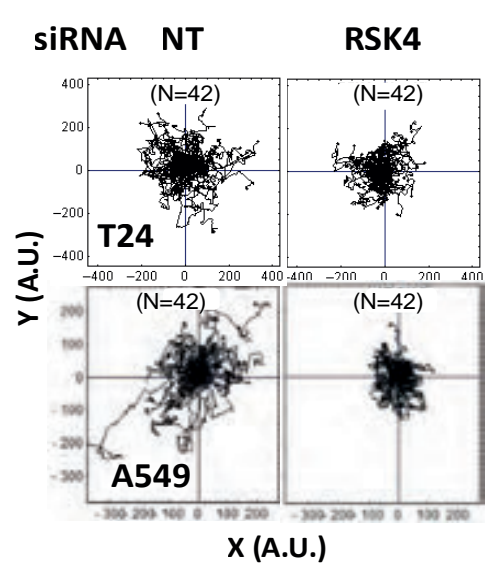

B.

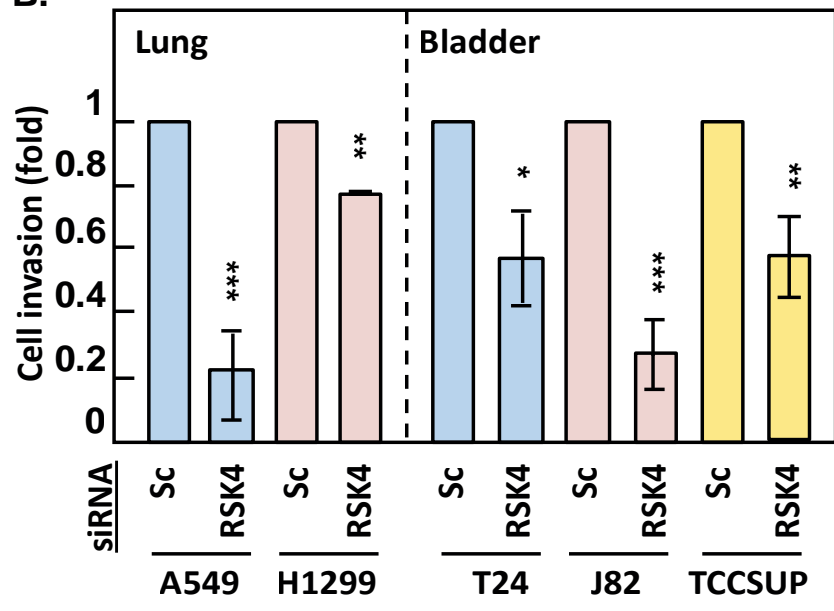

C.

NT

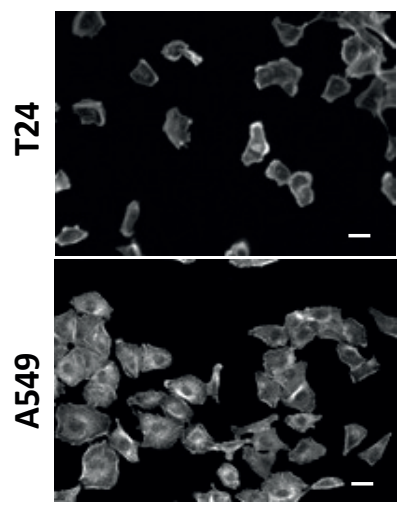

RSK4

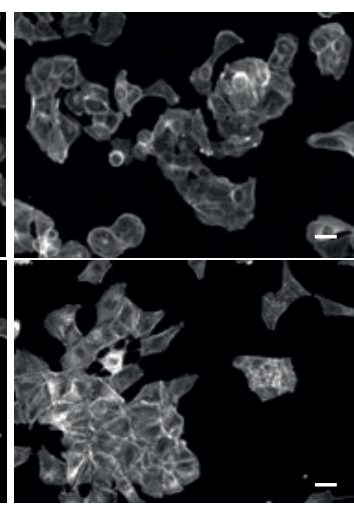

D.

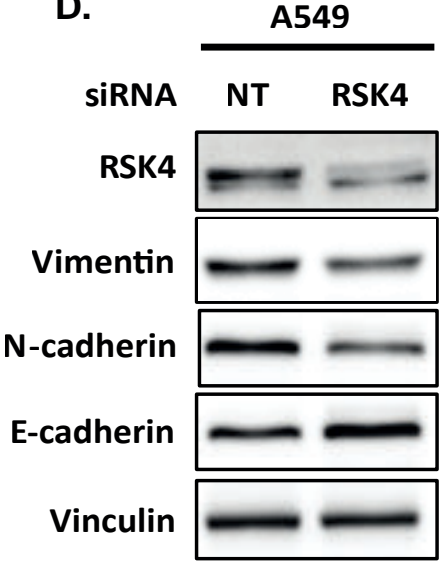

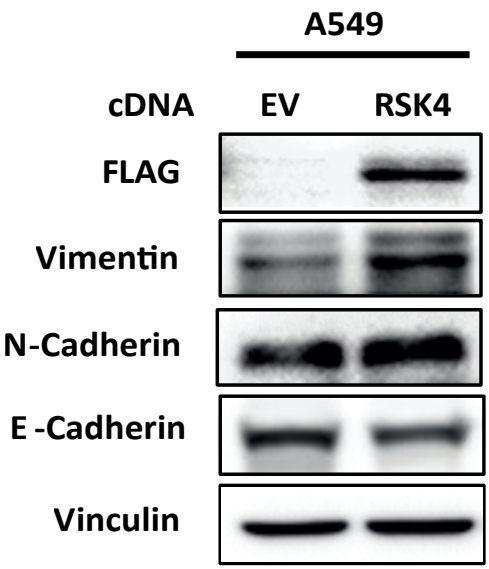

G.
E. TGGGGACTTTCCGC (5x)

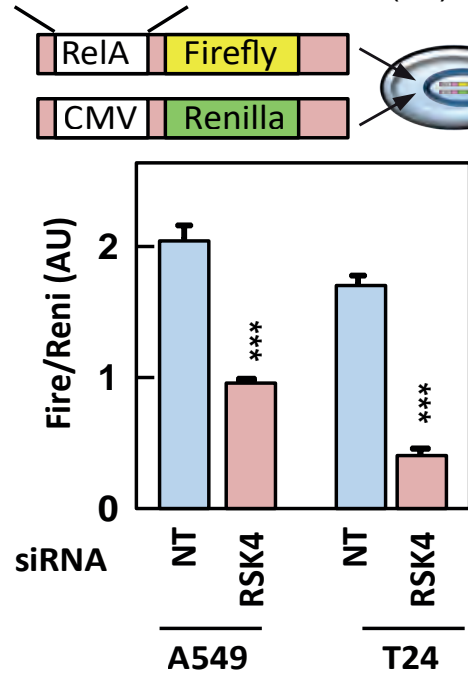

H.

P300-pSer89

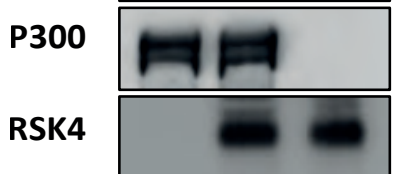

F.

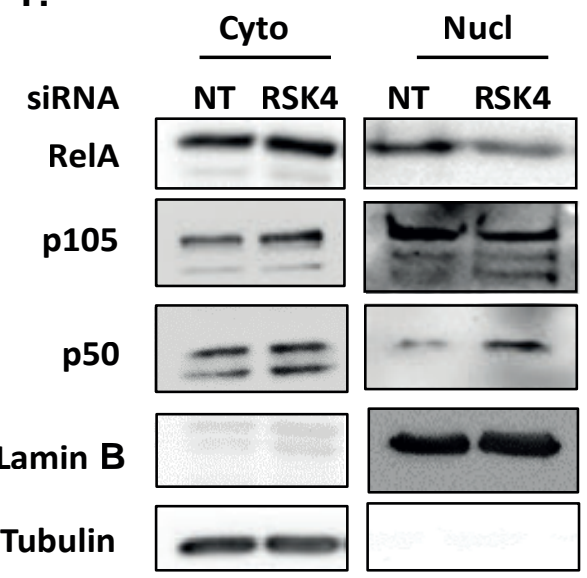

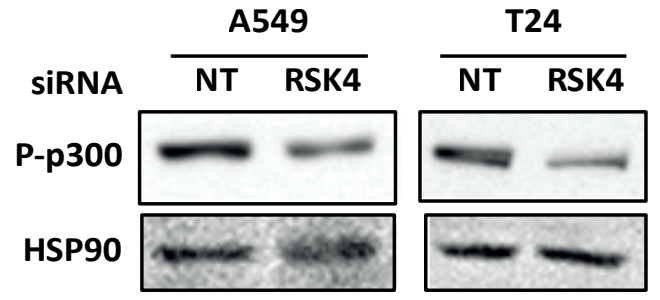
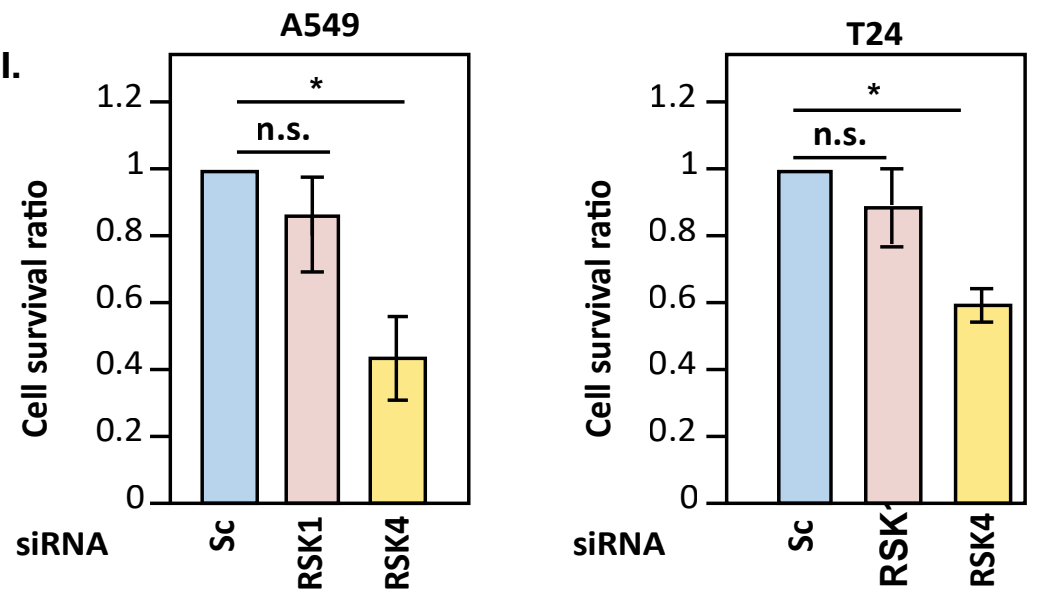
Fig 3

A.

Normal Lung Negative AdCa PositiveAdCa

Normal Tissue jary tumour
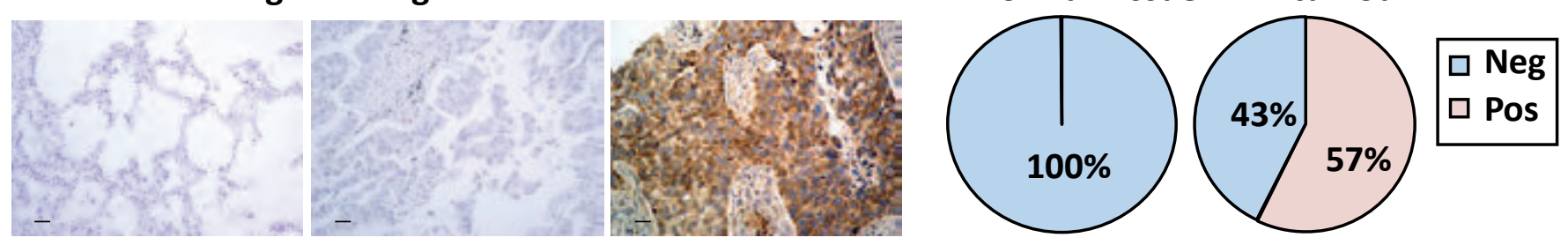

B.

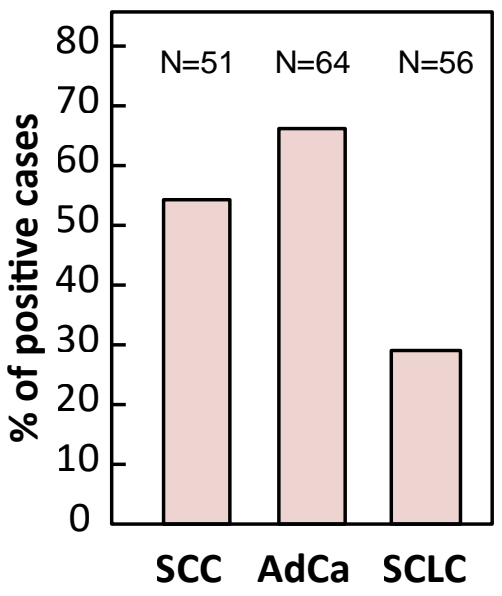

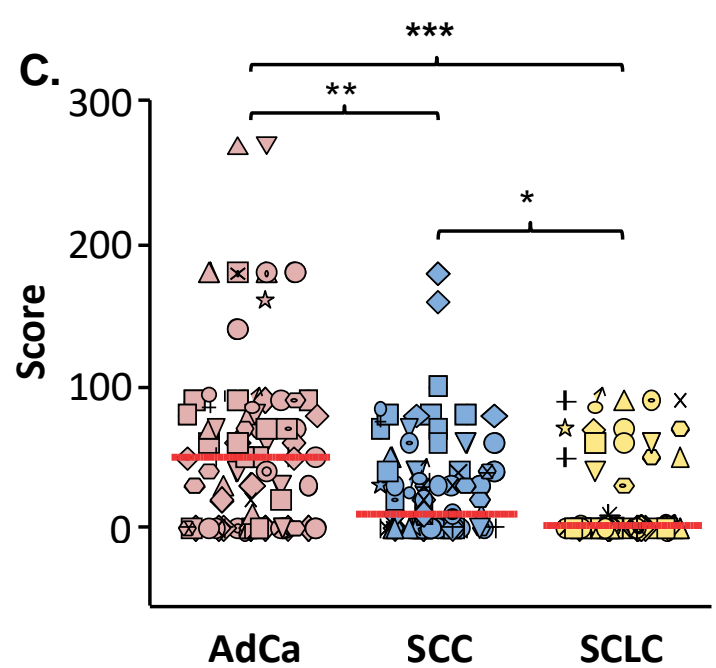

AdCa SCC SCLC

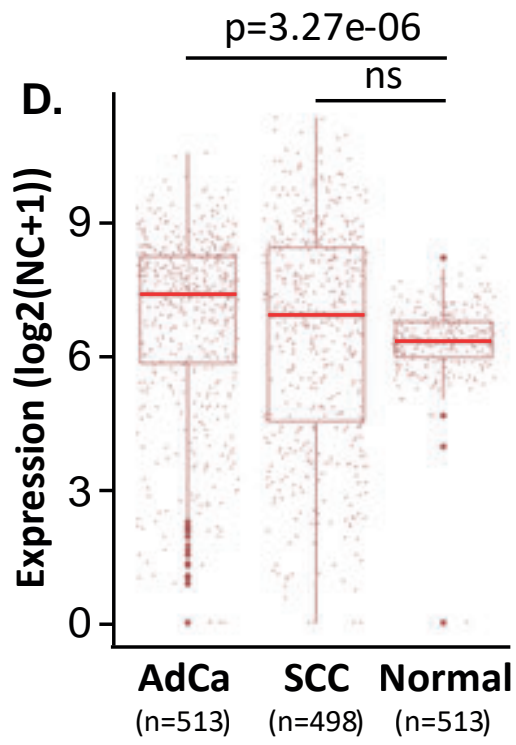

E.

AdCa: Probe 220737_at

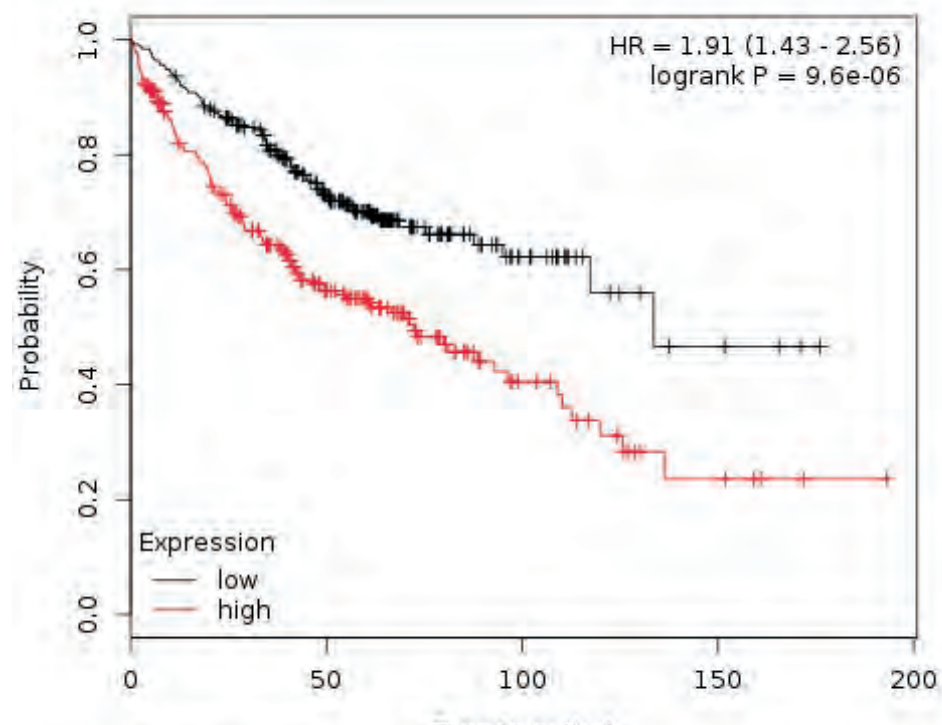

Time (months) Number at risk high 236

F.

\begin{tabular}{|l|c|c|c|c|}
\hline \multirow{2}{*}{ NSCLC subtype } & \multicolumn{2}{|c|}{ Cases (\%) } & \multicolumn{2}{c|}{ Average IHC score } \\
\cline { 2 - 5 } & Met > Primary & Met $\leq$ Primary & Met > Primary & Met $\leq$ Primary \\
\hline Adenocarcinoma & 52 & 48 & 55.23 & 43.43 \\
\hline Squamous cell carcinoma & 24 & 76 & 62.44 & 8.72 \\
\hline
\end{tabular}

SCC: Probe 220737_at

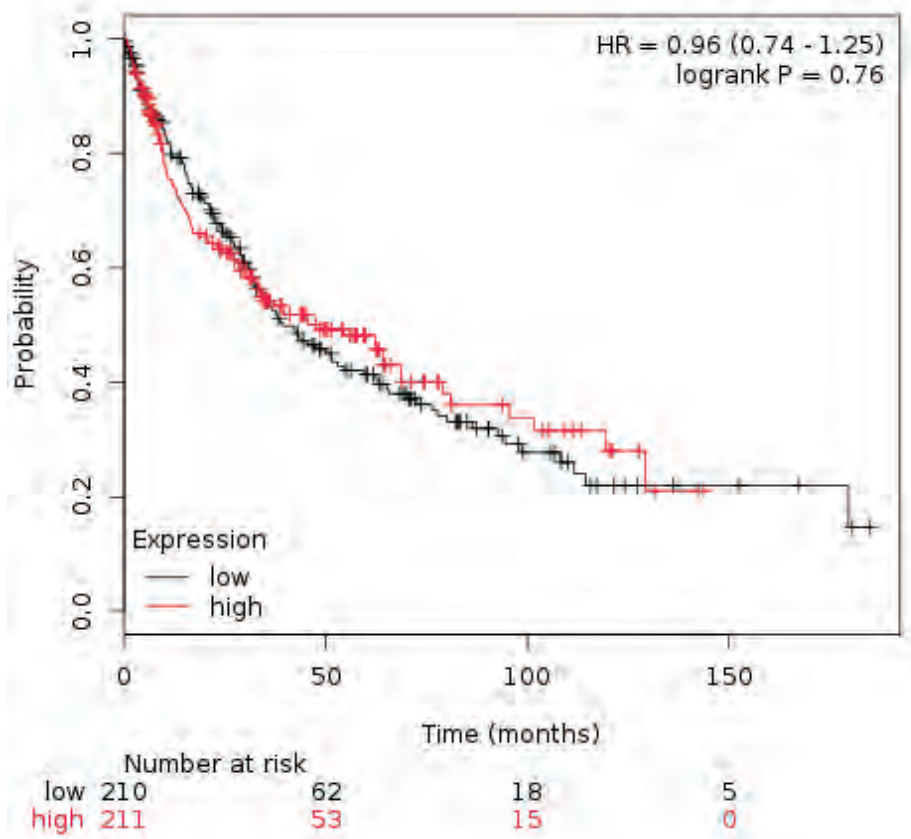


Fig 4

A.

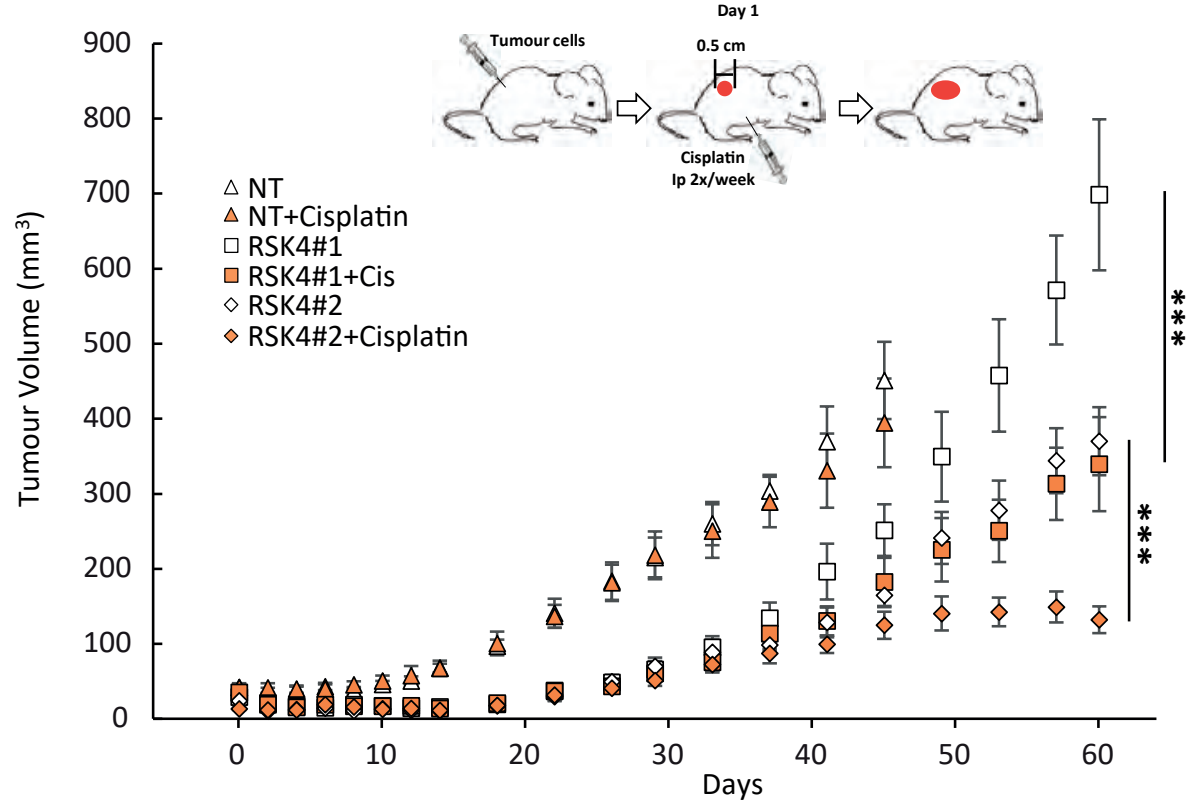

B.

C.
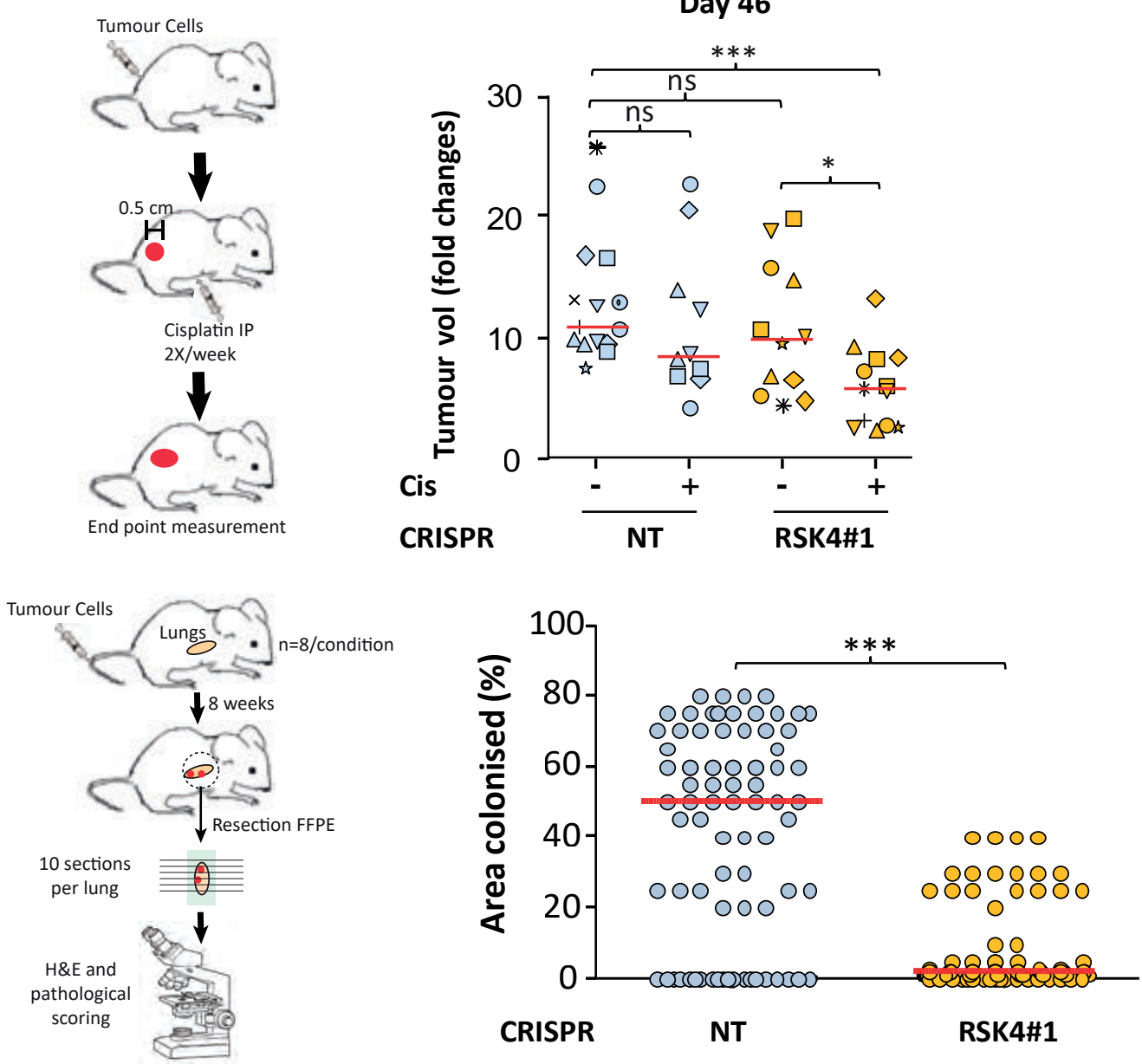

\section{Number of colonies}

\begin{tabular}{|c|c|c|c|c|c|c|c|c|c|c|c|c|c|c|c|c|c|c|c|c|c|c|c|}
\hline \multicolumn{2}{|c|}{ Section } & $\# 1$ & $\# 2$ & $\# 3$ & \#4 & $\# 5$ & $\# 6$ & $\# 7$ & $\# 8$ & \#9 & $\# 10$ & \multicolumn{2}{|c|}{ Section } & $\# 1$ & $\# 2$ & \#3 & \#4 & $\# 5$ & \#6 & \#7 & \#8 & \#9 & $\# 10$ \\
\hline \multirow{8}{*}{ 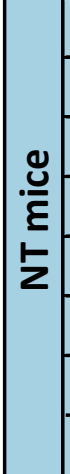 } & $\# 1$ & $>10$ & $>10$ & $>10$ & $>10$ & $>10$ & $>10$ & $>10$ & $>10$ & $>10$ & $>10$ & \multirow{2}{*}{\multicolumn{2}{|c|}{. }} & 4 & 4 & 4 & 4 & 4 & 5 & 5 & 5 & 5 & 5 \\
\hline & $\# 2$ & $>10$ & $>10$ & $>10$ & $>10$ & $>10$ & $>10$ & $>10$ & $>10$ & $>10$ & $>10$ & & & 1 & 1 & 1 & 1 & 1 & 1 & 0 & 0 & 0 & 0 \\
\hline & $\# 3$ & $>10$ & $>10$ & $>10$ & 10 & $>10$ & $>10$ & $>10$ & $>10$ & 10 & 10 & \multirow{5}{*}{ 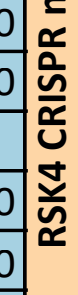 } & $\# 3$ & 1 & 1 & 1 & 1 & 0 & 0 & 0 & 1 & 1 & 1 \\
\hline & $\# 4$ & $>10$ & $>10$ & $>10$ & $>10$ & $>10$ & $>10$ & $>10$ & $>10$ & $>10$ & $>10$ & & $\# 4$ & 4 & 4 & 4 & 4 & 5 & 6 & 7 & $\epsilon$ & 5 & 5 \\
\hline & $\# 5$ & 0 & 0 & 0 & 0 & 0 & 0 & 0 & 0 & 0 & 0 & & $\# 5$ & 1 & 1 & 1 & 1 & 1 & 1 & 1 & 1 & 1 & 1 \\
\hline & $\# 6$ & $>10$ & $>10$ & $>10$ & $>10$ & $>10$ & $>10$ & $>10$ & $>10$ & $>10$ & $>10$ & & $\# 6$ & 2 & - & 2 & 2 & 0 & 0 & 0 & - & 0 & 0 \\
\hline & $\# 7$ & $>10$ & $>10$ & $>10$ & $>10$ & $>10$ & $>10$ & $>10$ & $>10$ & $>10$ & $>10$ & & \#7 & $\perp$ & 2 & 2 & 2 & 4 & 4 & 4 & J & o & 0 \\
\hline & \#8 & 0 & 0 & 0 & U & 0 & 0 & 0 & 0 & 0 & 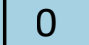 & & \#8 & 1 & 1 & $\perp$ & 1 & 0 & 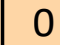 & 0 & U & 1 & 0 \\
\hline
\end{tabular}


Fig 5

A.

B.
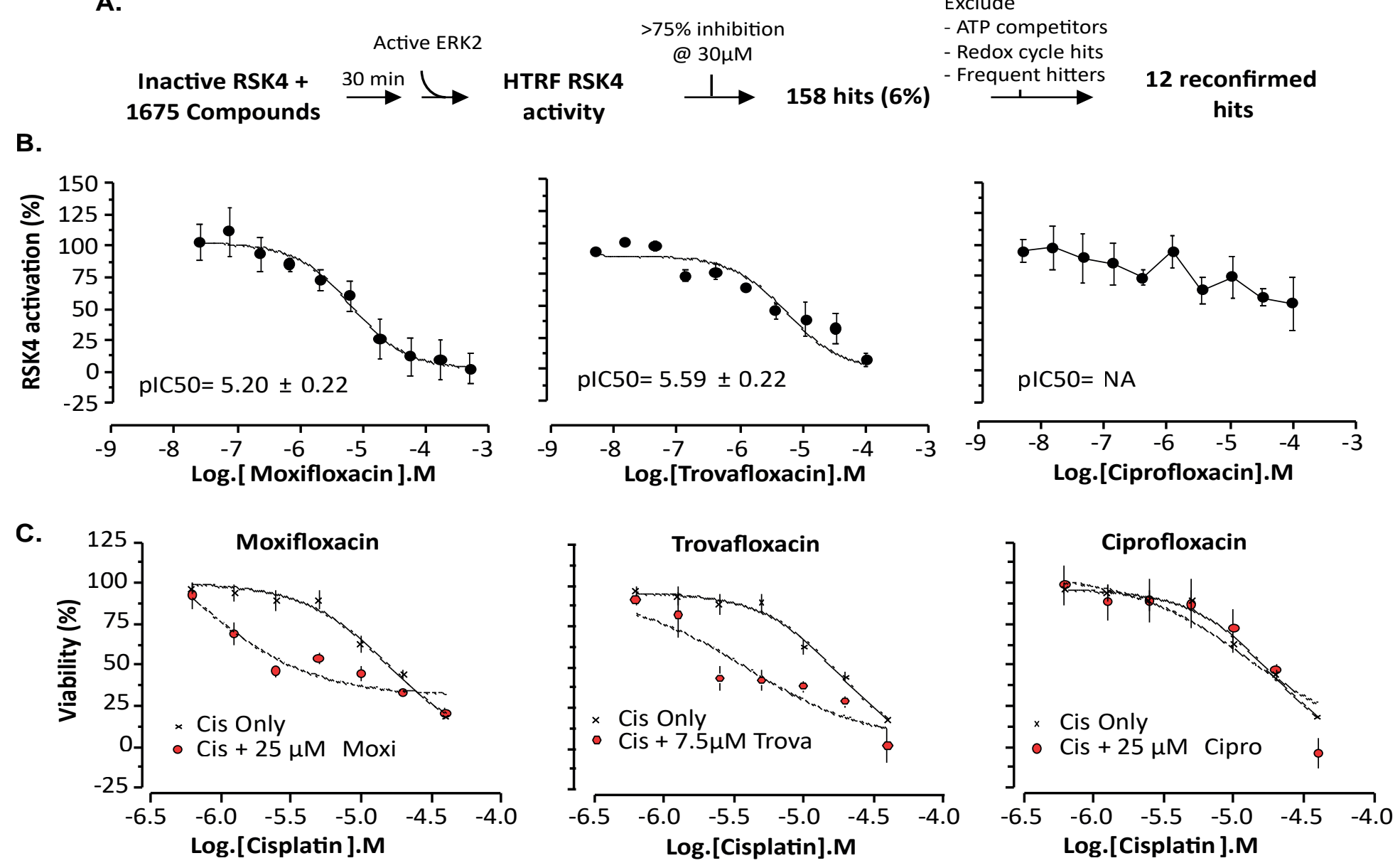

D.
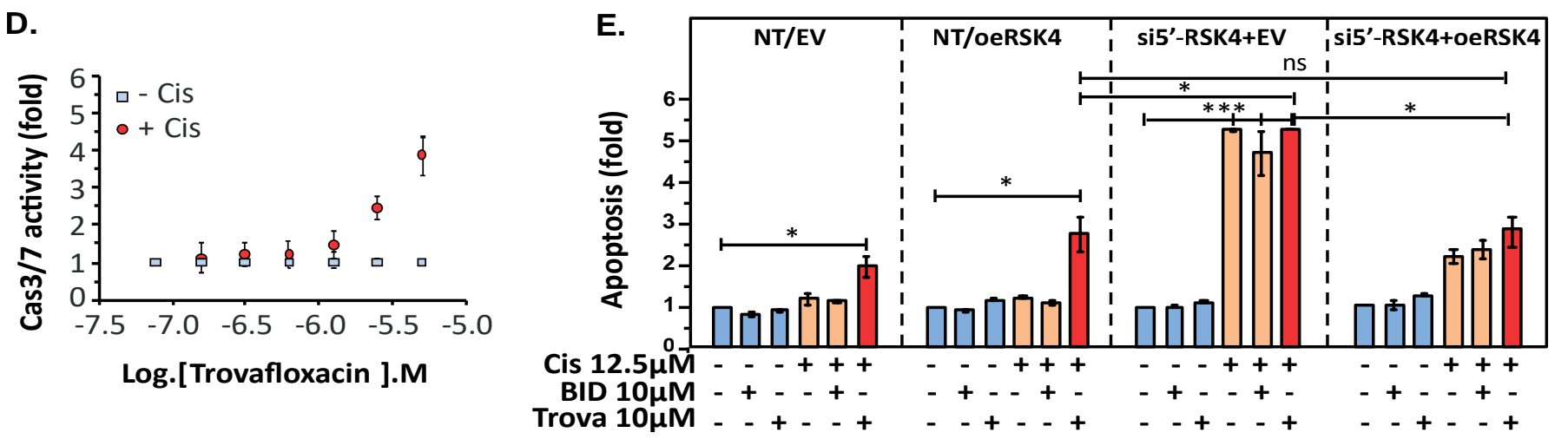

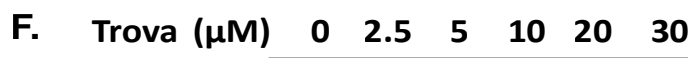
Vinculin $\mathrm{Wrar}$

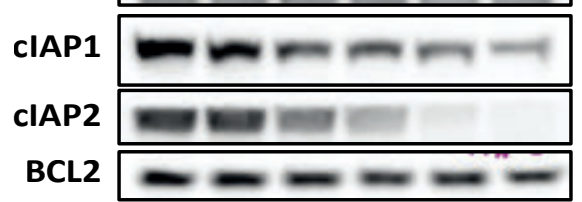

I.

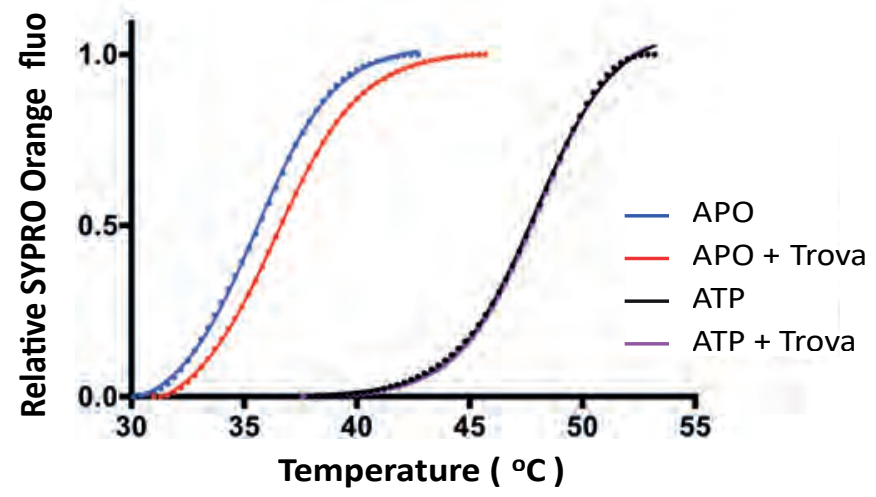

$\mathrm{H}$.
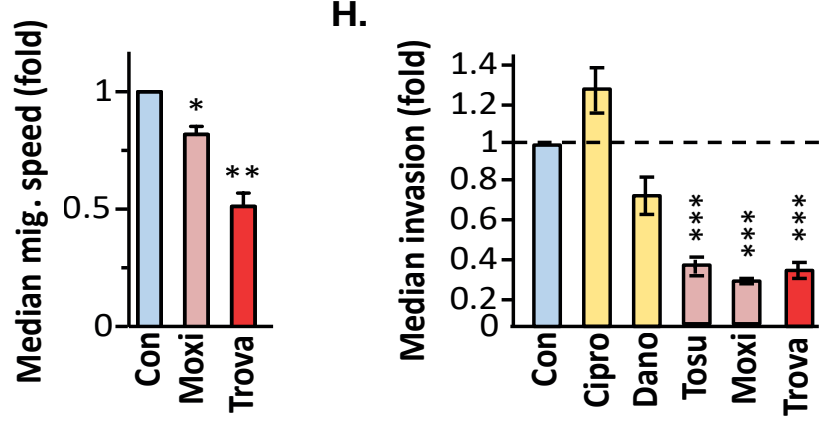

\begin{tabular}{|c|c|}
\hline Conditions & $\begin{array}{c}\text { RSK4 NTKD } \\
(3 \mu \mathrm{M}) \\
\operatorname{Tm}\left({ }^{\circ} \mathrm{C}\right)\end{array}$ \\
\hline APO & $35.52 \pm 0.02$ \\
APO+Trova $(100 \mu \mathrm{M})$ & $36.41 \pm 0.01$ \\
ATP $(1 \mathrm{mM})$ & $47.91 \pm 0.03$ \\
ATP $(1 \mathrm{mM})+\operatorname{Trova}(100 \mu \mathrm{M})$ & $48.01 \pm 0.02$ \\
\hline
\end{tabular}


Fig 6

A.

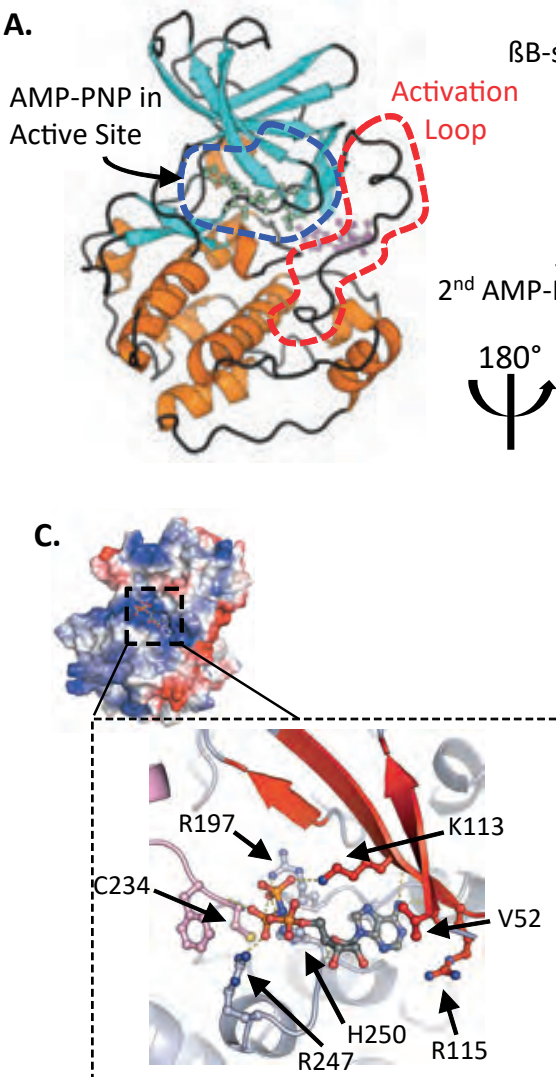

BB-sheet

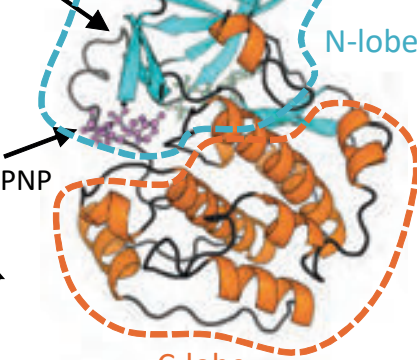

C-lobe

D.
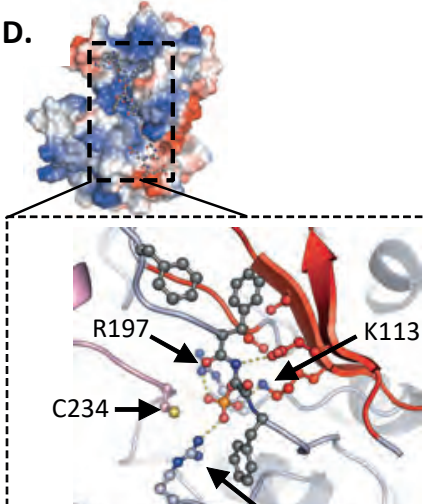

F.

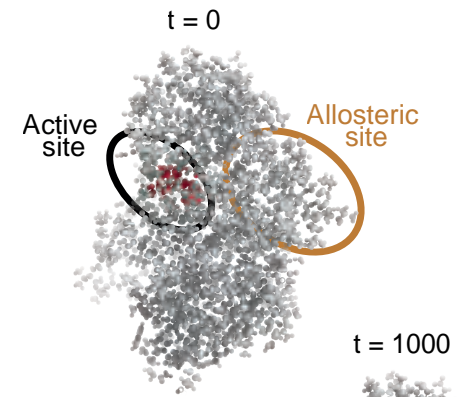

$\left(90^{\circ}\right)$

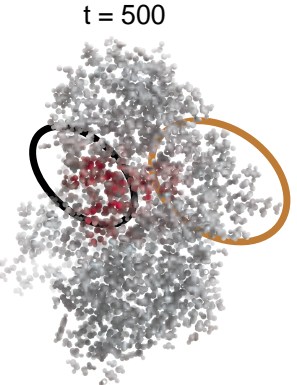

$\left(90^{\circ}\right)$

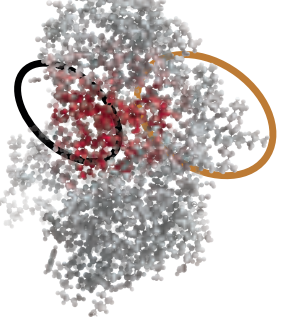

G.
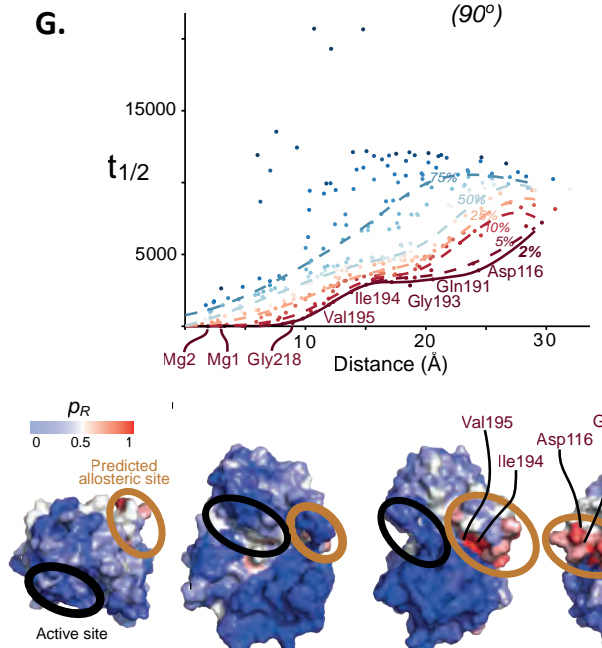

(top view)
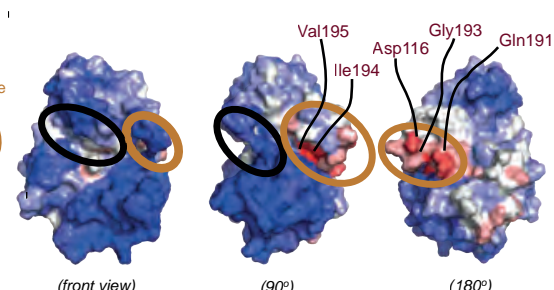

$\left(180^{\circ}\right)$

B.
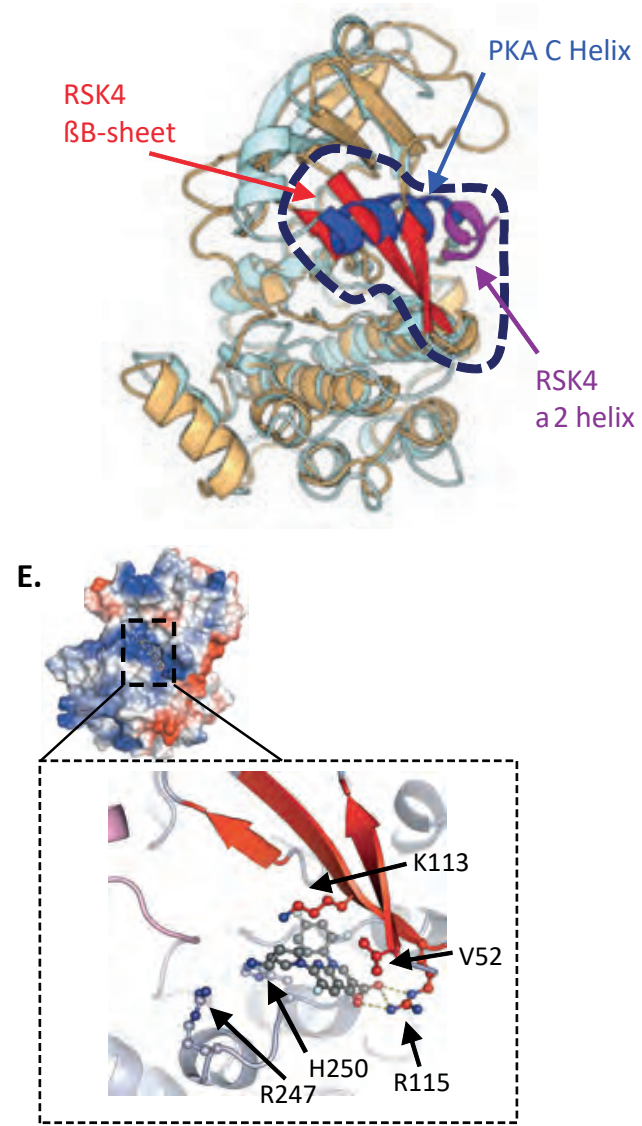
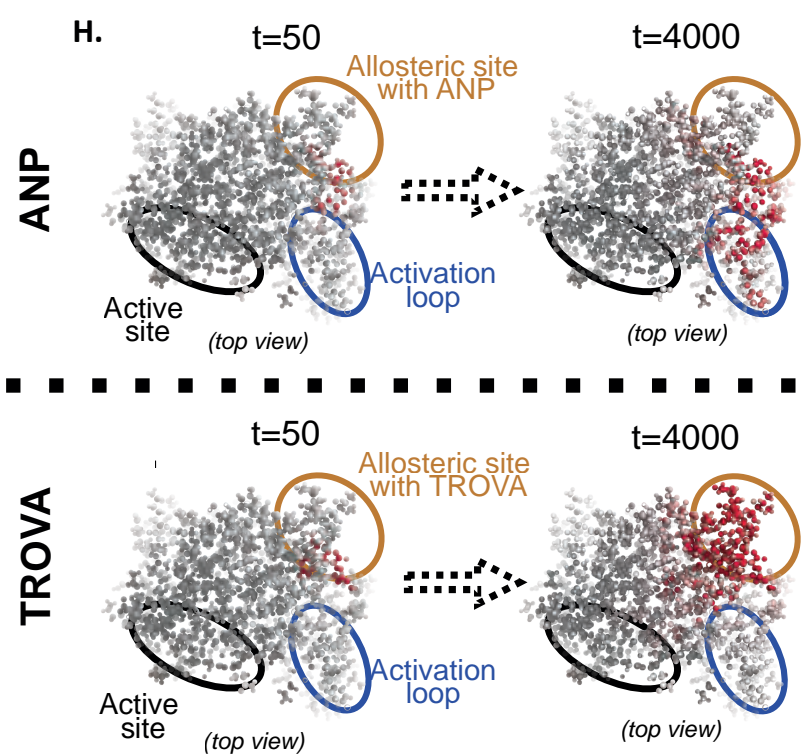

I.

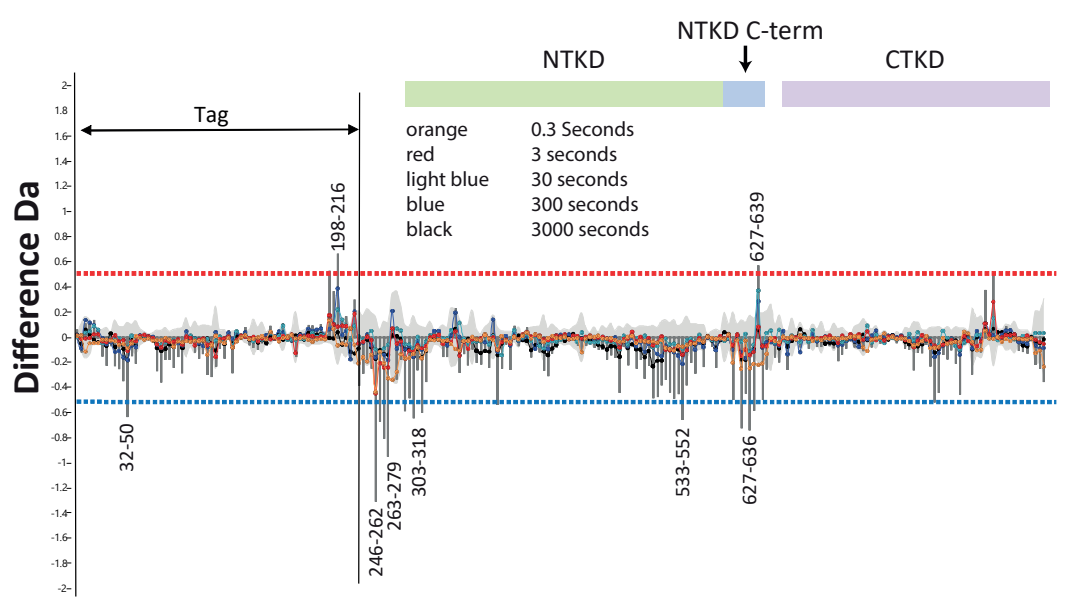


Fig 6

A. Active Site

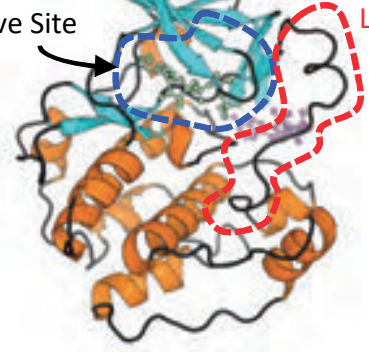

c.

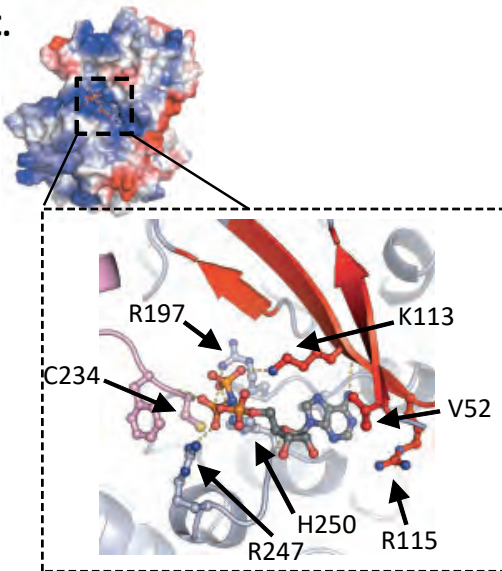

BB-sheet
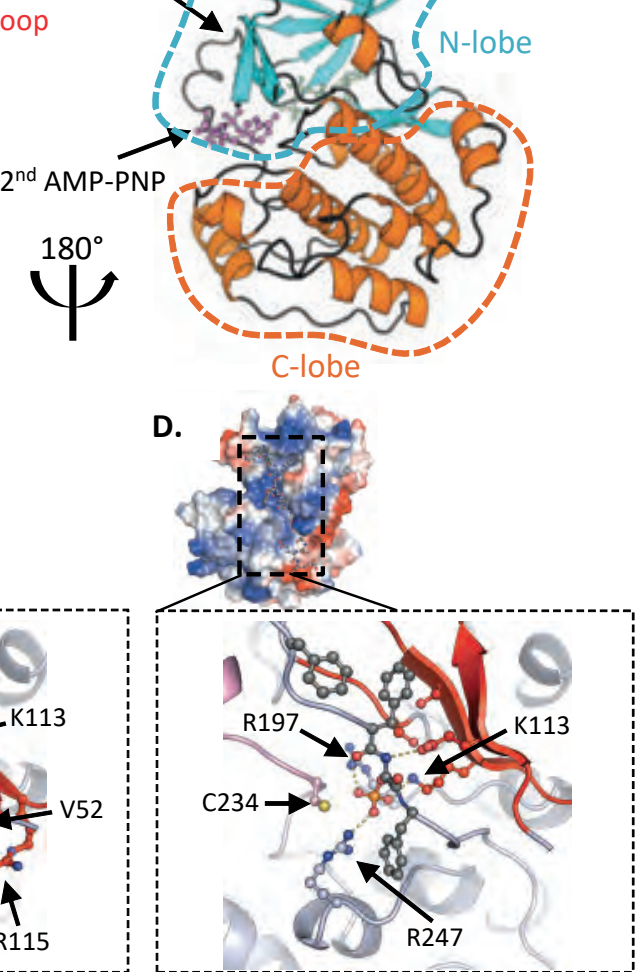

B.
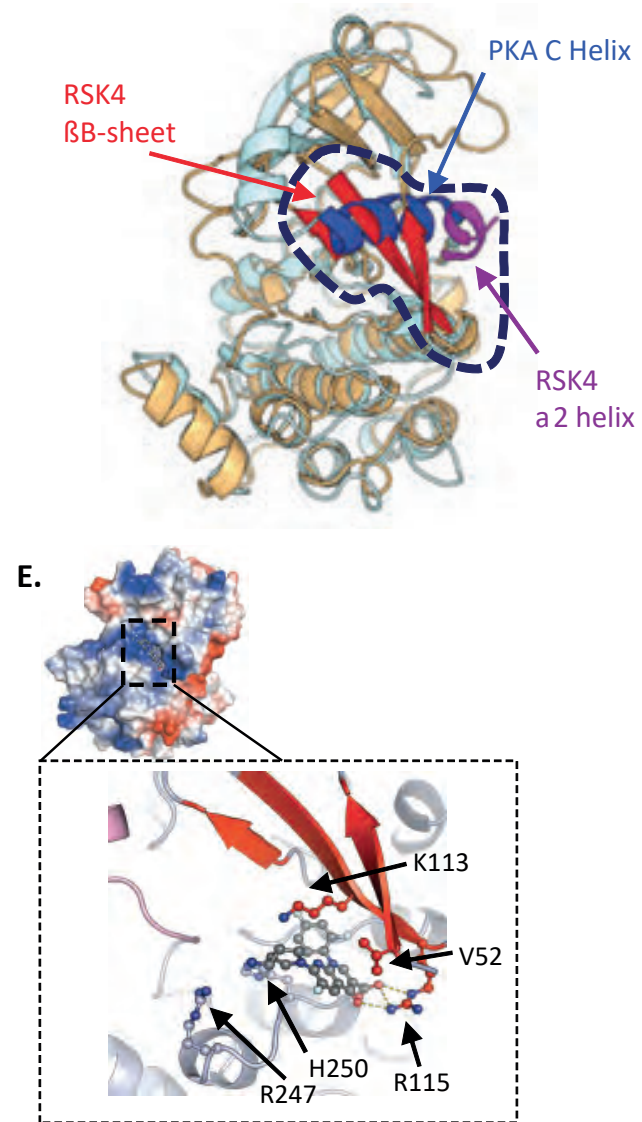

F.

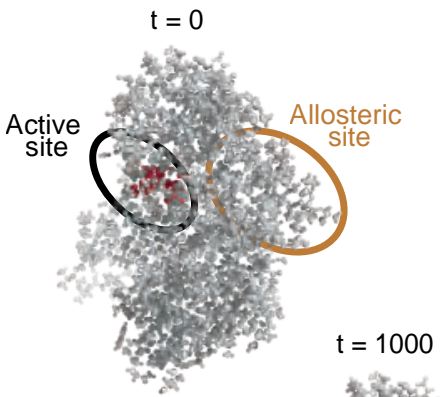

$\left(90^{\circ}\right)$

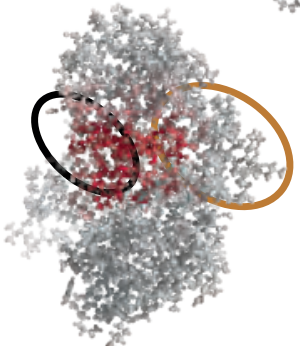

G.

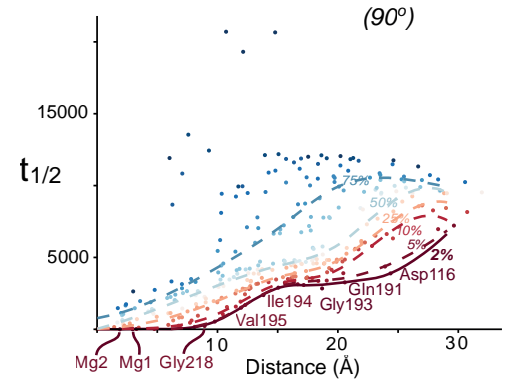

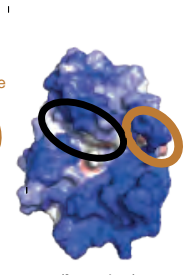

(tront view)
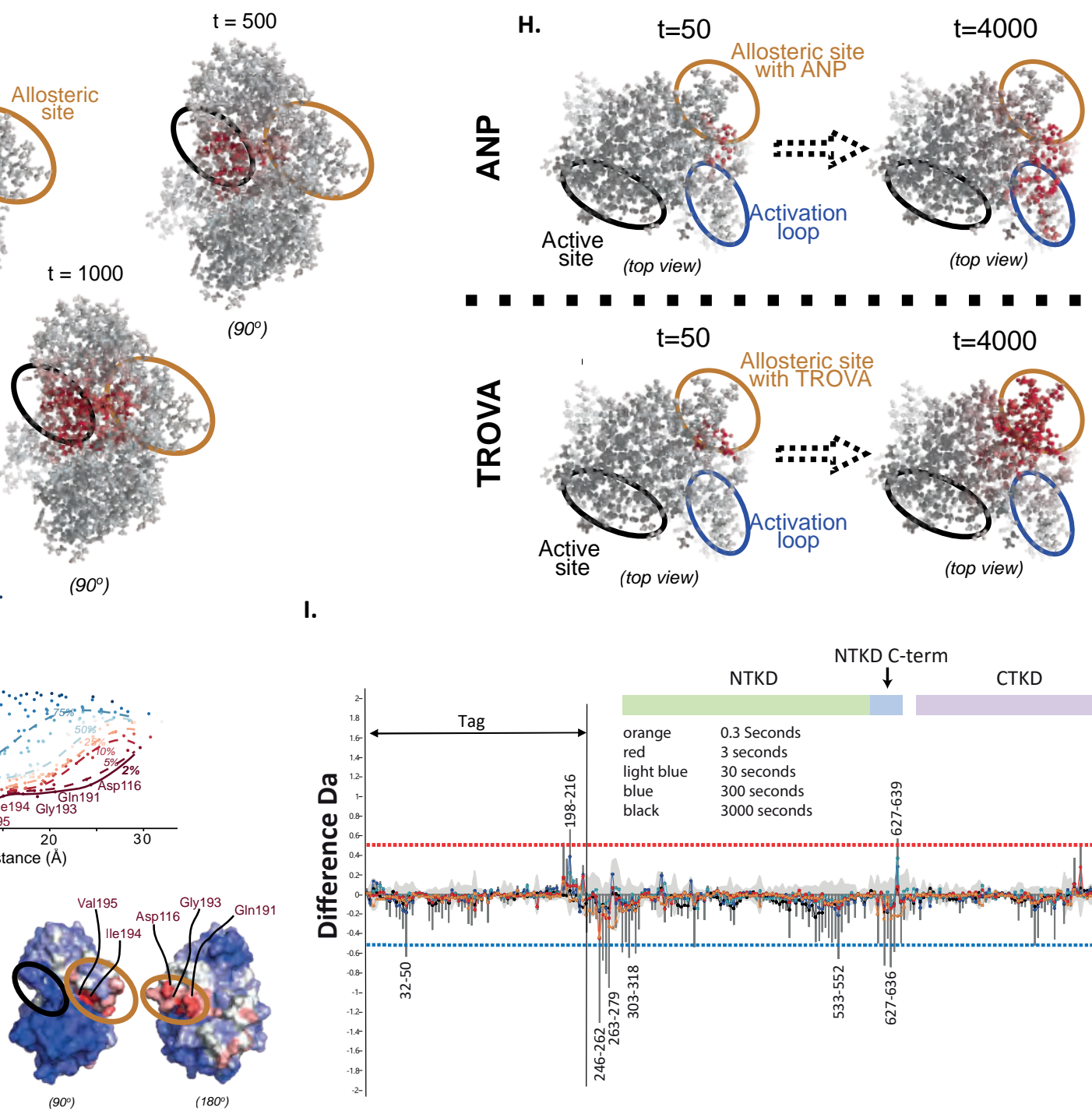

$\left(90^{\circ}\right)$

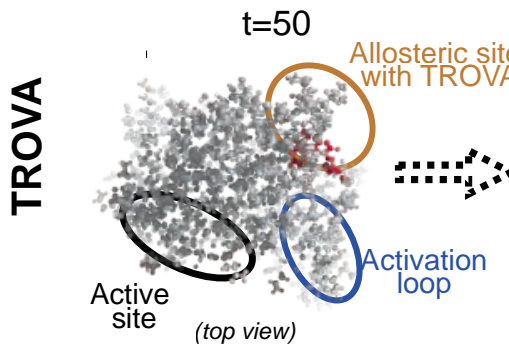

$t=4000$

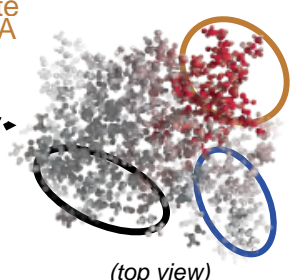

I.

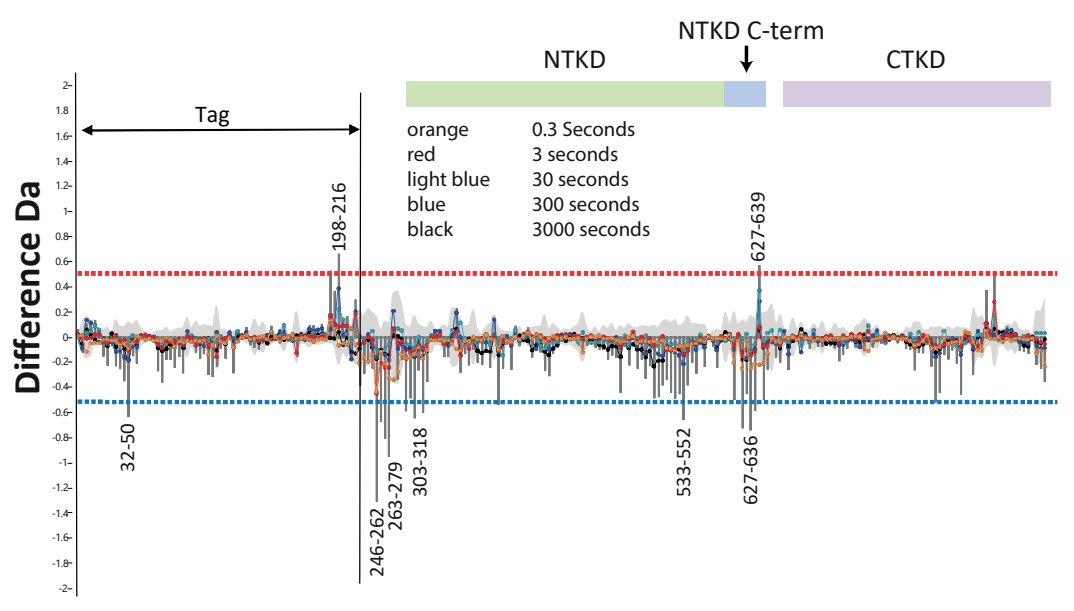


Fig 7

A.

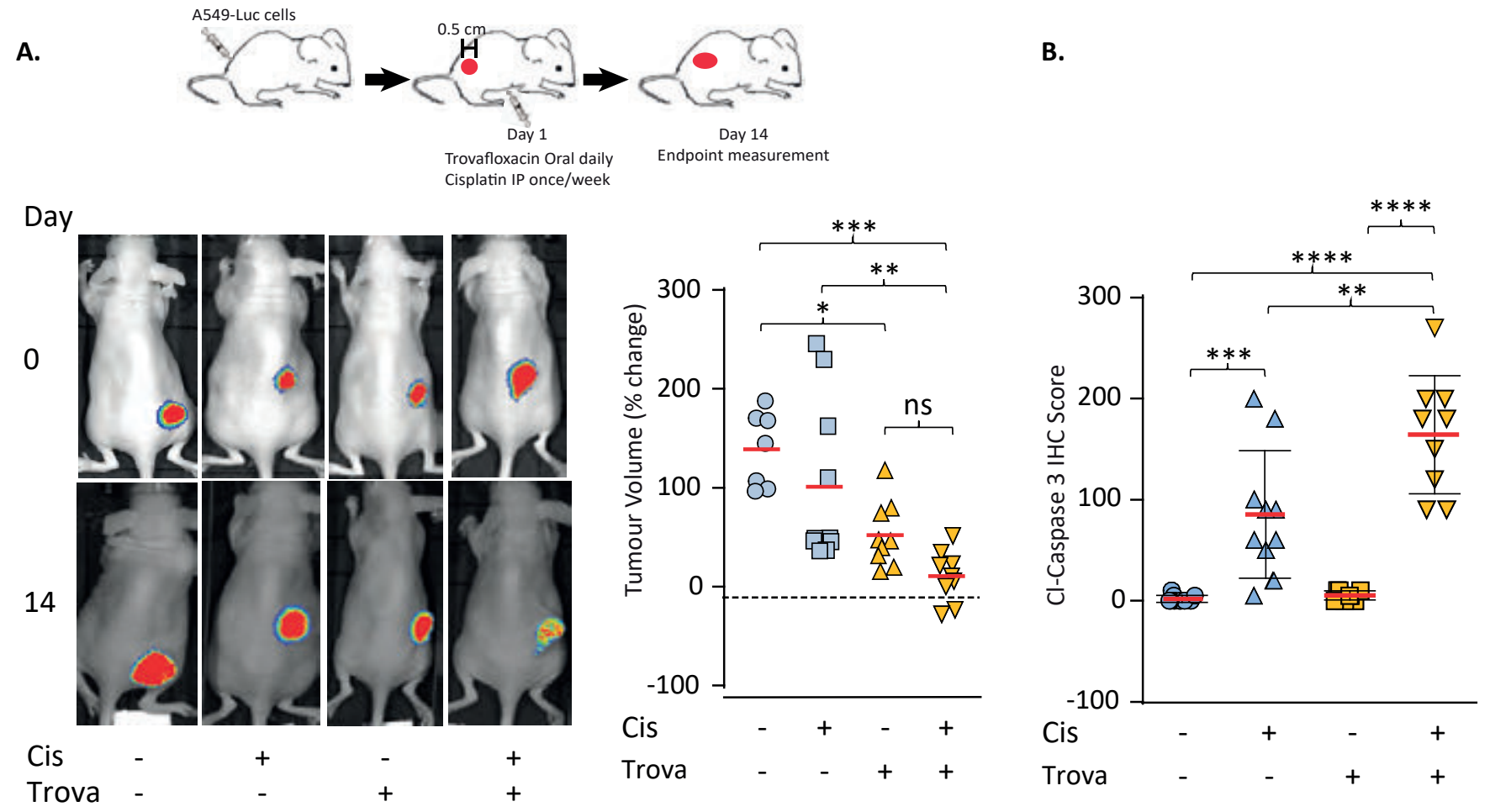

B.

c.

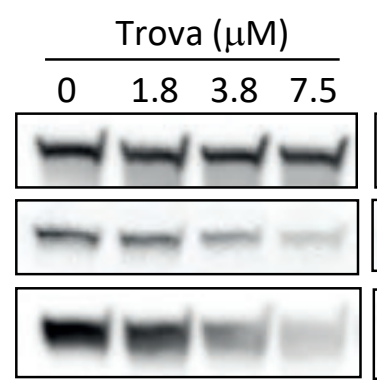

\begin{tabular}{cccc}
\multicolumn{4}{c}{ Cipro $(\mu \mathrm{M})$} \\
\hline 0 & 1.8 & 3.8 & 7.5
\end{tabular}

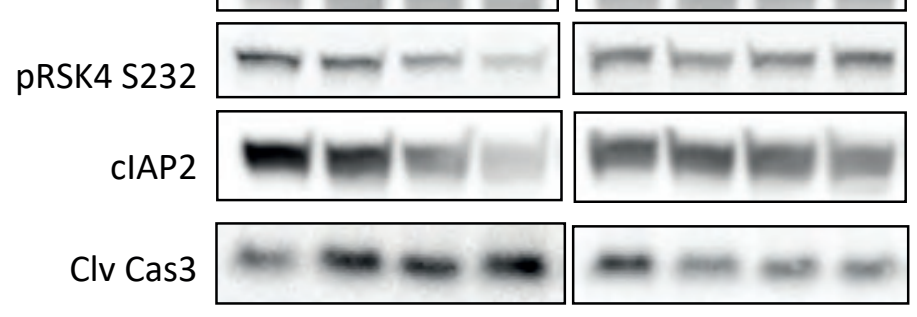

D.

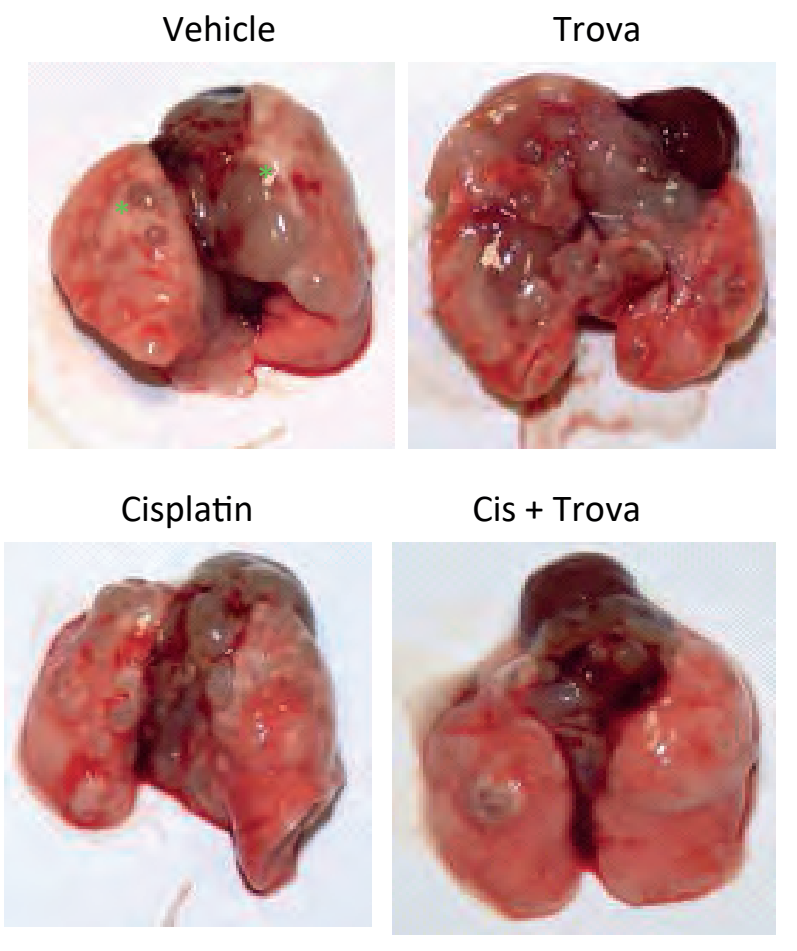

E.

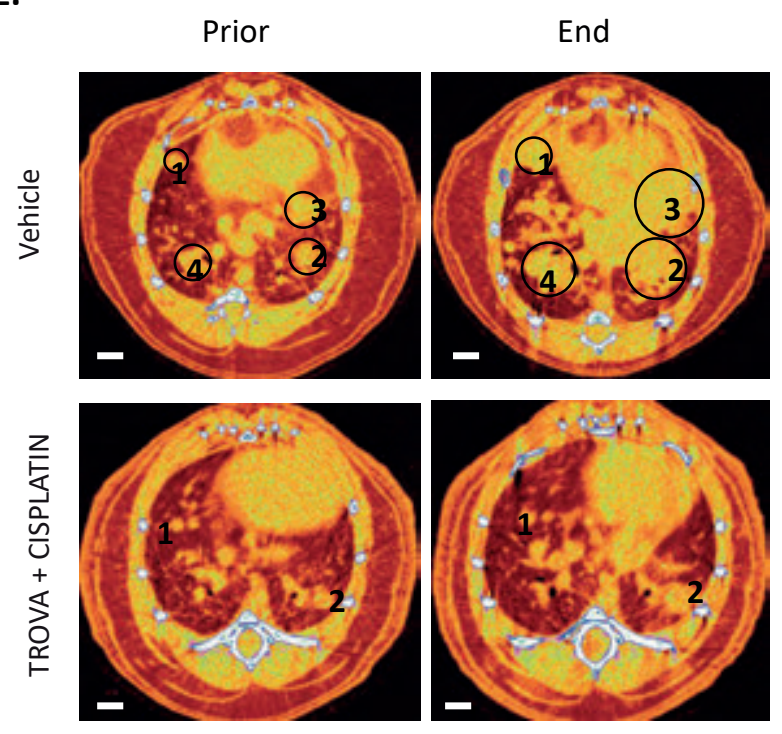

F.

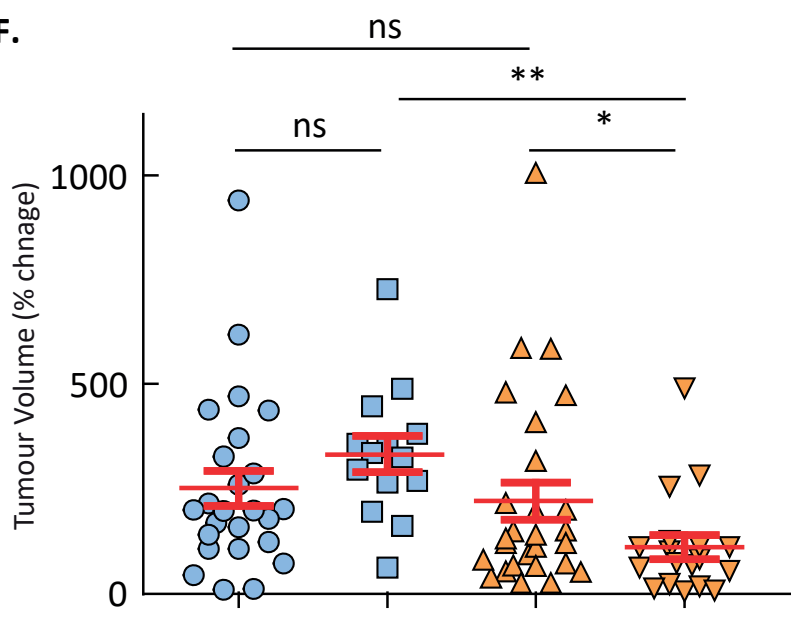

Cisplatin

Trova 


\section{SUPPLEMENTARY MATERIALS AND METHODS}

Cell lines and culture: Non-small cell lung cancer (A549, H1299, EKVX and HOP62) and bladder cancer (T24, TCCSUP and J82) cells were cultured in DMEM $/ 10 \%$ foetal bovine serum (FBS) at $37^{\circ} \mathrm{C}$, $10 \% \mathrm{CO} 2$.

RNA interference kinome screen: Cells were transfected with siRNA ( $35 \mathrm{nM}$ ) using Lipofectamine RNAi-Max (Invitrogen), according to the manufacturer's instructions, for $48 \mathrm{~h}$ to allow for target silencing prior to subsequent treatment. The Kinome siRNA library v2.0 (Qiagen) targeted 691 proteins with 4 individual oligonucleotide sequences/target. siRNAs targeting PLK1 were used as internal control for transfection efficiency across the screen. Luciferase-targeting (Dharmacon) oligonucleotides were used as negative controls. $48 \mathrm{~h}$ after transfection, cisplatin and taxol were added or not to the cells to achieve their IC50 of respectively $3.5 \mu \mathrm{M}$ and $40 \mathrm{nM}$. $48 \mathrm{~h}$ later, the plates were subjected to Crystal violet staining. Data were normalised to the readings obtained for the corresponding plate's Luciferase siRNA condition. Hit selection for targets sensitising or antagonising drug treatment was performed as previously (48). Validation experiments for screen hits used siRNAs from Dharmacon. Specific siRNA sequences used to target RSK4 can be found in table S2. Individual siRNAs transfections: 20nM siRNA oligonucleotides (Dharmacon) were transfected using Oligofectamine 3000 (ThermoFisher) according to the manufacturer's instructions. The siGENOME Non-targeting siRNA Pool \#2 (Dharmacon) was used as non-targeted control.

\section{Plasmid DNA transfection}

Cells were transfected with Lipofectamine 3000 (Invitrogen) following the manufacturer's instructions and used for experiments $24 \mathrm{~h}$ later when expression is maximal. The active RELA (T7RelA S536E - Plasmid \#24156 (49)), clAP1 and clAP2 (gifts from Jon Ashwell - plasmid \# 8311 and plasmid \# 8338, respectively(50)) plasmids were obtained from Addgene. RSK4 transcript variant I (NM_014496.5) ORF was PCR-amplified using RSK4-GFP as template (pLPS-3'EGFP backbone; Arizona State University plasmid repository, AZ, USA), and primers introducing a 5' Xhol restriction site prior to the start codon and a 3' EcoRI restriction site after the stop codon. Double digested PCR products (Xhol/EcoRI) were cloned into a pLPC vector containing a FLAG-HA-HA tag (FH2) at the N-terminus, kindly provided by deLange Lab (The Rockefeller University, NY, USA).

\section{Crystal violet staining}

Cells were fixed in $4 \%$ paraformaldehyde (PFA) and stained in $0.02 \%$ crystal violet solution for $15 \mathrm{~min}$. Plates were washed in water and air-dried. Crystal violet-precipitates were solubilised in $10 \%(\mathrm{v} / \mathrm{v})$ acetic acid (30 min, room temperature, gentle shaking) and absorbance measured at $595 \mathrm{~nm}$.

\section{Caspase $3 / 7$ activity assay}

The Caspase-Glo 3/7 Assay (Promega) was performed according to the manufacturers' instructions. Briefly, $100 \mu$ l of Caspase-Glo 3/7 reagent was added per 96-well and luminescence measured after 15 and 60 min of incubation at room temperature (PHERAstar microplate reader).

\section{Cell motility assay}

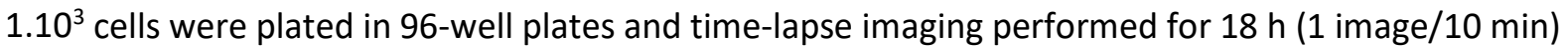
using a Zeis Axiovert microscope driven by Metamorph (Molecular Devices). Cells were tracked using Image $\mathrm{J}$ and tracks analysed in $\mathrm{R}$.

\section{Actin cytoskeleton staining}

Cells in 96-well plates were fixed with a 4\% PFA for 20 min, permeabilised with $0.1 \%$ Triton X-100 in PBS for 5 min, and blocked using 3\% Bovin Serum Albumine (BSA) in PBS. Actin was stained with Alexa Fluor 488-Phalloidin (Molecular Probe) and nuclear DNA revealed using DAPI (Molecular 
Probe). Images for 36 fields per well were acquired using an ImageXpress high-content imager (Molecular Devices).

qRT-PCR validation of target down regulation

Total cellular mRNA was extracted using Purelink RNA kit (Invitrogen) and converted into cDNA using High Capacity Reverse Transcriptase kit (Applied Biosystems). qRT-PCR was performed using Fast SYBR green master mix (Applied Biosystems) with gene specific primers on ABI $7900 \mathrm{HT}$ real-time PCR machine. HPRT and GAPDH were used as internal controls. For primer sequences used, see table S3.

\section{Western blotting}

Cellular proteins were extracted using a Radio immunoprecipitation assay buffer (RIPA) (50 mM Tris$\mathrm{Cl}, \mathrm{pH} 7.4,0.1 \%$ SDS, $0.1 \%$ sodium deoxycholate, $1 \%$ Triton $\mathrm{X}-100,150 \mathrm{mM} \mathrm{NaCl}, 2 \mathrm{mM}$ EDTA, $5 \%$ Glycerol supplemented with protease inhibitors cocktail tablets (Roche Diagnostics), $10 \mathrm{mM}$ $\beta$ Glycerophosphate, $1 \mathrm{mM}$ sodium orthovanadate, $10 \mathrm{mM}$ sodium fluoride). Equal protein amounts were diluted in $2 x$ Laemmli buffer, boiled for $5 \mathrm{~min}$ and analysed by SDS-PAGE/Western blotting using the relevant antibodies. For details of antibodies used, see table S4.

\section{In vitro kinase assay}

$1 \mu \mathrm{g}$ recombinant p300 (Abcam) was incubated with 200 ng recombinant RSK4 in Kinase Buffer (50 mM HEPES, $10 \mathrm{mM} \mathrm{MgCl2}, 2 \mathrm{mM}$ DTT, $5 \mathrm{mM} \beta$-glycerophosphate, $0.1 \mathrm{mM}$ Sodium orthovanadate). Reactions were started by adding $2 \mathrm{mM}$ ATP and pursued for 30 min before termination in SDSsample buffer.

\section{RelA reporter assays}

Cells were transfected with a RelA-driven firefly luciferase construct together with a CMV-driven Renilla luciferase construct used for normalisation of the firefly luciferase signal. Detection of luciferase activity was performed using the Dual-Glo luciferase kit (Promega) according to the manufacturer's protocol.

\section{Invasion assay}

$1.10^{4}$ cells were plated in 96 well-plates for $24 \mathrm{~h}$ prior to being covered with rat tail Collagen I (BD Bioscience) (3mg/ml final) in DMEM/1\% FCS. After $4 \mathrm{~h}, 25 \mu \mathrm{l}$ of DMEM/1\% FCS were placed above the gel. $25 \mu \mathrm{l}$ of DMEM/1\% FCS with $200 \mathrm{ng} / \mathrm{ml}$ EGF were added $24 \mathrm{~h}$ later. Cells were allowed to invade for $48 \mathrm{~h}$. The gels were fixed in 4\% PFA and cell nuclei revealed using Cytox Green (Molecular Probes). Nine stacks of $210 \mu \mathrm{m}$ ( $4 \mu \mathrm{m}$ intervals) were acquired per well with a Zeiss 7.10 invert confocal microscope. Stacks were analysed with ImageJ.

\section{Anoikis assay}

Cells transfected in 96-well plates with the relevant siRNA for $48 \mathrm{~h}$ were trypsinised and the content of each well split equally between an ultra-low attachment and a normal-adhesion 96-well plates (Corning). $18 \mathrm{~h}$ later, the content of the ultra-low attachment plate was transferred to a normaladhesion 96-well plate and left to adhere prior to Crystal violet staining. The absorbance of plates where cells underwent detachment were then compared to that of those where cells had remained adherent.

\section{Immunohistochemistry (IHC)}

Post-mortem homemade tissue microarrays (TMA) containing syngenic primary tumour/metastasis samples from 98 patients was prepared (Ethic Ref: 06/Q0406/154). For full details on this TMA, see Supplementary Methods. Commercial TMAs (LC10010, LC1006, LC1201) containing 64 AdCa, 51 SCC, 56 SCLC and 25 normal lung samples were purchased from US Biomax Inc. IHC was performed using a 
RSK4 antibody (Sigma Prestige) at 1/50 dilution. A semi-quantitative IHC score was used as previously described (51). Signal specificity was determined using RSK4-silenced paraffin-embedded A549 cells (fig. S4A).

In vitro recombinant kinases assay screen

Trovafloxacin $(10 \mu \mathrm{M})$ was tested in an in vitro kinase assay using a panel of tyrosine and serine/threonine kinases as previously published (52).

\section{Generation of CRISPR RSK4 A549 cells}

The protocol used for the CRISPR/Cas9 system was as reported earlier (53). The backbone vector pX458, pSpCas9(BB)-2A-GFP was a gift from Feng Zhang (Addgene plasmid \#48139). Two guide RNA sequences were designed to target RSK4 gene (5'-CCCTATTACTCATCATGTTAAGG-3' on Exon 3 and 5'-TGGGCAGCTCTATGCAATGAAGG-3' on Exon 4). Forward and reverse oligonucleotides for each were annealed and phosphorylated using T4 DNA Ligase Reaction Buffer and T4 Polynucleotide Kinase (New England Biolabs) at $37^{\circ} \mathrm{C}$ for $30 \mathrm{~min}$ followed by $95^{\circ} \mathrm{C}$ for $5 \mathrm{~min}$. pX458 was digested using Bbsl (Thermo Scientific, Yokohama, Japan) at $37^{\circ} \mathrm{C}$ for $2 \mathrm{hrs}$, and gel purification was performed using a QIAquick Gel Extraction Kit (QIAGEN). The ligation reactions of pX458 and the annealed oligonucleotides were performed at room temperature for $4 \mathrm{hrs}$ using a T4 DNA ligase Kit (New England Biolabs). Then, the ligated products were purified using PlasmidSafe exonuclease (Cambio) at $37^{\circ} \mathrm{C}$ for $30 \mathrm{~min}$. The plasmids were transformed into Stbl3, and amplified using Qiagen maxiprep kit. Each plasmid was transfected into A549 cells by electroporation using Amexa nucleofector II according to the manufacturer's protocol. The transfectants were selected using puromycin prior to isolation of clones. Cell lines were tested for knockdown of RSK4 using Western blotting and the best clones selected for further experiments.

\section{Post-mortem tissue micro array}

The Royal Postgraduate Medical School archives were searched for metastatic lung cancer (LC) samples. The reports of 12,580 post-mortem (PM) examinations performed at the Hammersmith Hospital between January 1970 and December 2005 were reviewed. LC was identified in 499 cases. Patients who received anticancer therapy for their malignancy, as assessed through review of PM reports and medical notes, were excluded $(n=286)$. In total, formalin-fixed paraffin embedded (FFPE) tissue blocks pertaining to 213 patients were retrieved. These patients were further screened for stringent tissue quality criteria, including (1) confirmed histopathologic diagnosis of metastatic LC; (2) lack of significant autolytic degeneration of the target tissues; (3) complete PM dissection of all the organs; and (4) PM interval <24 $\mathrm{h}$ from death.

Histotype classification as well as metastatic distribution was confirmed following review of newly cut Haematoxylin and Eosin (H\&E) sections by a consultant pulmonary pathologist (FAM). The suitability of both primary and metastatic deposits to immunohistochemical (IHC) studies was confirmed following preliminary immunostaining for two pan-cytokeratin markers: MNF116 (Dako) incubated at 1:200 concentration for $1 \mathrm{~h}$ and CK Cam 5.2 (BD Biosciences) incubated at 1:20 concentration for $1 \mathrm{~h}$ after $0.1 \%$ trypsin in phosphate buffered saline incubation for $10 \mathrm{~min}$. PM specimens showing dubious or unsatisfactory MNF116/CK Cam 5.2 staining were discarded. Based on the combination of the above quality criteria, a total number of 98 cases were considered suitable to be included in the study. A tissue micro-array was constructed by obtaining three $1 \mathrm{~mm}$ cores from the most representative and better-preserved areas of the tumours and re-embedded in microarray blocks. 
This study protocol received full approval by an accredited Research Ethics Committee (Reference: 06/Q0406/154).

\section{Survival analysis \\ Univariate overall survival analysis for lung adenocarcinoma or lung squamous carcinoma patients was performed on microarray datasets using an online Kaplan-Meier plotter (kmplot.com) and patients stratified by their low or high expression status for the corresponding RSK isoform using the median as a cut-off expression value. Probes used for the analysis were as follow: RSK1; 203379_at, RSK2; 203843_at, RSK3; 212912_at and RSK4; 220737_at and 220738_s_at.}

\section{Interrogation of public datasets for RSK expression}

Comparison of RSK isoform mRNA expression in cancer: lung and bladder cancer data were extracted from the The Cancer Genome Atlas (TCGA) pan-cancer dataset downloaded from the UCSC Xena Browser. Analysis was performed in R with plots created using the lattice package. Comparison of RSK isoforms mRNA expression between cancer and normal tissue: For lung, the same TCGA dataset as above was used. Analysis was performed in $\mathrm{R}$ and plots created using the lattice package. For bladder, the GEO RNA-Seq dataset GSE133624 was analysed in R and two previously published microarray datasets (54)' (55) were analysed in Oncomine (www.oncomine.org).

\section{Crystallization}

Proteins were concentrated to $10 \mathrm{mg} / \mathrm{mL}$ only in the presence of AMP-PNP (Sigma-Aldrich), as previously reported for RSK2 NTKD (56). $1 \mu$ I RSK4 NTKD or RSK4 NTKD S232E was mixed with $1 \mu$ of precipitant solution (20\% PEG 6000, 0.1 M TRIS pH8, 2 mM ZnCl2). $1 \mu$ I RSK4 NTKD pS232 was mixed with $1 \mu$ l of precipitant solution (18\% PEG 6000, $0.1 \mathrm{M}$ TRIS pH8, $2 \mathrm{mM} \mathrm{ZnCl2).} \mathrm{Crystals} \mathrm{were} \mathrm{grown}$ within 1 day at $18{ }^{\circ} \mathrm{C}$ by the sitting drop vapour diffusion method.

\section{Data collection and structure determination}

The crystals were flash-cooled in liquid nitrogen after a short soaking in cryo-protection solution (40\% (w/v) glycerol, $20 \%$ PEG 6000, $0.1 \mathrm{M} \mathrm{TRIS} \mathrm{pH8,} 2 \mathrm{mM} \mathrm{ZnCl}$ ) and then frozen by plunging into liquid nitrogen. X-ray diffraction data were collected at Diamond Light Source beamlines 104-1, 103 and 124 respectively for RSK4 NTKD, RSK4 NTKD pS232 and RSK4 NTKD S232E. Data were processed using XDS (57). The structures were determined by molecular replacement using the program PhaserMR (CCP4) (58) using respectively RSK2 NTKD structure (3G51) (56) for RSK4 NTKD and RSK4 NTKD for RSK4 NTKD pS232 and S232E. The structures were refined using Refmac5 (59) and PHENIX.refine (60). PHENIX.Xtriage (60) found 1 pseudo-merohedral twin operator in RSK4 NTKD pS232 and S232E and a twin law h,-k,-I was applied during refinement with a PHENIX.refine. Once a satisfactory description of the protein electron density was complete, water molecules and AMPPNP were added using PHENIX.refine and PHENIX.LiganFit, respectively (60). Model building was performed using the graphics program Coot (61). Figures were prepared using PyMOL (DeLano Scientific). Coordinates have been deposited with the Protein Data Bank: RSK4 NTKD (6G77), RSK4 NTKD pS232 (6G76) and RSK4 NTKD S232E (6G78).

\section{Docking}

Molecular docking simulations were performed with HADDOCK web docking software (62), using RSK4 NTKD structure and a phosphorylated peptide in random coil covering the sequence of the hydrophobic motif between residues 380 and 398 (NAHQLFKGF-pS-FVATSIAEE). AutoDock Vina (63) (64) was used for docking simulation of trovafloxacin sarafloxacin, tosufloxacin tosylate, ciprofloxacin and clinafloxacin on RSK4 NTKD structure using a docking box of $17 \times 17 \times 17 \AA$ (gridpoint spacing of $1 \AA \AA$ ). Optimal box size was estimated according to the radius of giration of trovafloxacin (65). Binding energy, electrostatic energy, the number of possible conformation(s) of the ligand-receptor complex and also predicted polar bonds were obtained from Vina. 
Trovafloxacin was prepared using the "LigPrep" (Schrödinger Release 2020-2: LigPrep, Schrödinger, LLC) module of Schrodinger before molecular docking. Specifically, the ligand was minimized using the OPLS3 force field, the possible ionization states were created at $\mathrm{pH} 7.0 \pm 2.0$, the tautomers were generated, specific chiralities of the ligands were retained, and a total of 12 conformations were generated relative to the indefinite chiralities. The docking box was generated using the "Receptor Grid Generation" (66) module of Schrodinger, and was placed in a comparable position as the AutoDock Vina box. All parameters were default. Trovafloxacin was docked into the allosteric surface of RSK4 NTKD using an extra precision (XP) algorithm of Glide (66). The pose with the best score was rescored using Prime (Schrödinger Release 2020-2: Prime, Schrödinger, LLC) MM-GBSA approach (67). The top-ranked pose was selected.

Pymol (The PyMOL Molecular Graphics System, Version 2.0 Schrödinger, LLC. http://ww.pymol.org) was used to visualize the results.

\section{Differenctial scanning fluorimetry (DSF)}

DSF was performed using the fluorescent dye Sypro Orange (Sigma Aldrich) and a DNA Engine Opticon 2 (Bio-Rad) to monitor binding of Trovafloxacin and Ciprofloxacin to the RSK4 NTKD. The fluorescence intensity was plotted as a function of temperature and the curve's inflection point (Tm) was calculated by fitting the experimental data with a Boltzman equation in PRISM (GraphPad Software).

\section{Circular dichroism}

Far UV CD spectra were recorded on a Chirascan spectrometer (Applied Photophysics Ltd) equipped with a thermostated cell holder. Far UV measurements were performed at $20^{\circ} \mathrm{C}$ in $15 \mathrm{mM} \mathrm{NaCl}$ using protein concentrations of 3.51, 5.08 and $6.37 \mu \mathrm{M}$ respectively for RSK4 NTKD, RSK4 NTKD pS232 and RSK4 NTKD. The spectra were recorded in fused silica cuvettes of $1 \mathrm{~mm}$ path length. Five scans were averaged. Spectral decomposition for secondary structure predictions was achieved through DichroWeb (68) (69) using SELCON method.

\section{HDX-MS experiments}

Hydrogen/deuterium exchange reaction: Deuterium exchange reactions of Rsk4 (100 uM), and Rsk4 (100 uM) with Trvoa (300 uM), were initiated by diluting the proteins in D2O (99.8\% D2O ACROS, Sigma) in 25mM Tris- $\mathrm{HCl}$ pH8.0, $150 \mathrm{mM} \mathrm{NaCl}, 5 \mathrm{mM}$ TCEP and 4\% DMSO to obtain a final D2O percentage of $\sim 92 \%$. For all experiments, deuterium labelling was carried out at $23^{\circ} \mathrm{C}$ (unless otherwise stated) at five time points, $3 \mathrm{~s}$ on ice, $3 \mathrm{~s}, 30 \mathrm{~s}, 300 \mathrm{~s}$, and 3,000 s in triplicate. The labelling reaction was quenched by the addition of chilled $2.4 \%$ (vol/vol) formic acid in $2 \mathrm{M}$ guanidinium hydrochloride, to rapidly lower the $\mathrm{pH}$. Protein samples were then immediately snap frozen in liquid nitrogen and stored at $-80^{\circ} \mathrm{C}$ prior to analysis.

UPLC-HDX-MS: The quenched protein samples were rapidly thawed and subjected to proteolytic cleavage with pepsin followed by reversed phase HPLC separation. Briefly, the protein was passed through an Enzymate BEH immobilized pepsin column, $2.1 \times 30 \mathrm{~mm}, 5 \mu \mathrm{m}$ (Waters) at $200 \mu \mathrm{L} / \mathrm{min}$ for $2 \mathrm{~min}$, the peptic peptides were trapped and desalted on a $2.1 \times 5 \mathrm{~mm} \mathrm{C18} \mathrm{trap} \mathrm{column} \mathrm{(Acquity}$ BEH C18 Van-guard pre-column, $1.7 \mu \mathrm{m}$, Waters). Trapped peptides were subsequently eluted over $11 \mathrm{~min}$ using a $3 \%-43 \%$ gradient of acetonitrile in $0.1 \% \mathrm{v} / \mathrm{v}$ formic acid at $40 \mu \mathrm{L} / \mathrm{min}$. Peptides were separated on a reverse phase column (Acquity UPLC BEH C18 column $1.7 \mu \mathrm{m}, 100 \mathrm{~mm} \times 1 \mathrm{~mm}$ 
(Waters, UK) and detected on a SYNAPT G2-Si HDMS mass spectrometer (Waters) over a $\mathrm{m} / \mathrm{z}$ of 300 to 2000 , with the standard electrospray ionization (ESI) source with lock mass calibration using [Glu1]-fibrino peptide $B(50 \mathrm{fmol} / \mu \mathrm{L})$. The mass spectrometer was operated at a source temperature of $80^{\circ} \mathrm{C}$ and a spray voltage of $2.6 \mathrm{kV}$. Spectra were collected in positive ion mode. Peptide identification was performed by MSe (70) using an identical gradient of increasing acetonitrile in $0.1 \% \mathrm{v} / \mathrm{v}$ formic acid over $11 \mathrm{~min}$.

Data analysis: The resulting MSe data were analyzed using Protein Lynx Global Server 3.0 software (Waters, UK) with an MS tolerance of $5 \mathrm{ppm}$. Mass analysis of the peptide centroids was performed using DynamX 3.0 software (Waters). Only peptides with a score $>6.4$ were considered. The first round of analysis and identification was performed automatically by the DynamX 3.0 software, however, all peptides (deuterated and non-deuterated) were manually verified at every time point for the correct charge state, presence of overlapping peptides, and correct retention time.

Deuterium incorporation was not corrected for back-exchange and represents relative, rather than absolute changes in deuterium levels. Changes in H/D amide exchange in any peptide may be due to a single amide or a number of amides within that peptide. The DynamX 3.0 software plots the standard deviation for every peptide. The error band shows the standard deviation of the plotted uptake or difference for each peptide. When there are multiple exposures, as in this experiment, for a given peptide, the maximum standard deviation is plotted for each peptide. A sigma multiplier of 1 is applied to the standard deviation to produce the gray error bar plotted in Fig. 61 and fig. S12B.

\section{Auto-phosphorylation assay}

$10 \mu \mathrm{M}$ non-phosphorylated RSK4 NTKD was incubated at room temperature in $25 \mathrm{mM} \mathrm{HEPES} \mathrm{(pH}$ 7.5), $150 \mathrm{mM} \mathrm{NaCl}, 10 \mathrm{mM} \mathrm{MgCl} 2,5 \mathrm{mM}$ DTT, $5 \%$ Glycerol, supplied with $2 \mathrm{mM}$ ATP alone or in presence of $100 \mu \mathrm{M}$ peptide covering the sequence of the hydrophobic motif between residues 380 and 398 (HP: NAHQLFKGFSFVATSIAEE) (Alta Bioscience Ltd), or $100 \mu \mathrm{M}$ of the phosphorylated peptide (pHP: NAHQLFKGF-pS-FVATSIAEE) (Alta Bioscience Ltd) for $30 \mathrm{~min}, 1 \mathrm{~h}$ and $2 \mathrm{~h}$. Samples were immediately diluted with $2 x$ Laemmli buffer, boiled for $5 \mathrm{~min}$ and analysed by $4-12 \%$ Bis-Tris SDS-PAGE gel and Western blotting using anti-Phospho-Rsk-1/2/3/4 (S221/227/S218/232) antibody (St John's Laboratory) and ECL (Millipore).

\section{Animal experiments}

All studies were performed under a UK Home office approved project license and in accordance with institutional welfare guidelines. Mice were randomized by tumour burden. Analysis of $\mathrm{Ct}$ scan images to measure changes in tumour volume was done blinded. Animals were treated without knowledge of anticipated outcomes. No data points were removed as outliers.

Xenograft experiments: For tumour growth experiments, $5 \times 10^{6}$ CRISPR-NT, CRISPR-RSK4 A549 or A549-Luc cells were injected subcutaneously into the flanks of female nu/nu-BALB/c mice. Tumour measurements were performed daily by caliper and volumes calculated using $V=($ length $(\mathrm{mm})) \mathrm{x}$ (width $(\mathrm{mm})) \times($ width $(\mathrm{mm})) \times \pi / 6$. When tumours reached $50 \mathrm{~mm}^{3}$, treatment $\pm 3.5 \mathrm{mg} / \mathrm{kg}$ cisplatin in PBS (intraperitoneally weekly or twice weekly) and $30 \mathrm{mg} / \mathrm{kg}$ trovafloxacin by daily oral gavage. For metastatic experiments, CRISPR-NT and CRISPR-RSK4 A549 cells ( $1 \times 10^{6}$ in $\left.100 \mu \mathrm{l}\right)$ were tail veininjected into SCID mice and tumours left to develop for 2 months. The animals were then sacrificed and their lungs extracted, fixed and embedded into paraffin. 10 microtome slices over a depth of $600 \mu \mathrm{m}$ were analysed microscopically for the presence of tumour nodules following H\&E staining.

\section{KRASV12/TP53-/- (KP)-driven genetically engineered mouse model:}

For autochthonous tumour formation, lung tumours were initiated using intratracheal intubation of $1 \times 10^{6} \mathrm{pfu}$ (KrasLSL-G12D/+: Trp53flox/flox mice) adenovirus expressing Cre-recombinase (Gene Transfer Vector Core) in mice between 6-12 weeks of age. Mice were treated 14 weeks after adenoCre infection with vehicle, trovafloxacin $(175 \mathrm{mg} / \mathrm{kg}$ ) 5 days per week by oral gavage, cisplatin ( 5 $\mathrm{mg} / \mathrm{kg}$ ) weekly by i.p injection or combined trovafloxacin $(175 \mathrm{mg} / \mathrm{kg}) 5$ days per week by oral 
gavage and cisplatin ( $5 \mathrm{mg} / \mathrm{kg}$ ) weekly by i.p injection for 3 weeks. Tumour volume was measured by micro-CT analysis using the SkyScan 1176. Micro-CT data were processed and reconstructed using NRecon (SkyScan). Reconstructed data were subsequently imaged using DataViewer and tumour volumes were calculated using the CTan program (SkyScan). Trovafloxacin was freshly prepared in sterile water before o.g. administration. Cisplatin was freshly solubilised in DMSO and diluted into PBS before i.p. administration.

\section{Tumour explant drug sensitivity assay}

Single drugs and combinations of cisplatin $(25 \mu \mathrm{M})$ and trovafloxacin $(40 \mu \mathrm{M})$ were tested on patientderived bladder tumour explants as in (71).

\section{Generation of SIGNIFICANT trial data}

Details of the design and analysis of the SIGNIFICANT trial is published elsewhere (31). In summary the trial randomised 1565 patients receiving cyclical chemotherapy for solid tumours or lymphoma between August 1999 and April 2003, who were at risk of temporary severe neutropenia $(<500$ neutrophils per cubic millimetre) to receive either levofloxacin $500 \mathrm{mg}$ once daily or placebo for seven days during the expected neutropenic period, for up to six chemotherapy cycles. The primary outcome was the incidence of clinically documented febrile episodes attributed to infection and overall survival time was not collected as part of the original design.

Ethical approval was obtained for the conduct of the SIGNIFICANT Trial (West Midlands Multi-centre Research Ethics Committee (MREC) reference 99/7/08). Patients provided written informed consent at trial entry but this did not include permission for long term follow-up or documenting date of death. Subsequently, the original MREC advised that it was acceptable to trace date of death without further consent. Dates of death were obtained through the NHS Strategic Tracing Service (NSTS) who receive notification via the NHS central register when a death is registered and issue a formal notification. It advised that the vast majority of deaths in England and Wales are listed within 6 weeks. Participants' full name and date of birth were entered into the database and the patient was either confirmed as alive or date of death was displayed. Survival data from Scotland were obtained by sending patient information to the General Register Office for Scotland (GROS) which conducted patient tracing. The GROS advises that deaths are generally registered within 2 weeks. A small number of patients were traced through hospital records and matched using hospital number, date of birth and name. Initial patient tracing through NSTS was conducted between August 2007 and January 2008, but in order to ensure at least five years follow-up at the time of tracing, this was repeated in late July 2008 where it was needed. Tracing was conducted through GROS during late May 2008.

Survival time was calculated from trial entry until date of death from any cause. Any traced patients who had no date of death were assumed to be alive 6 weeks prior to tracing and a censored survival time was calculated using this endpoint. Patients alive at the time of tracing were censored at July $1^{\text {st }}$ 2007 for the first tracing set, June $1^{\text {st }} 2008$ for the second set and April $1^{\text {st }} 2008$ for patients in Scotland. For patients who could not be traced, date last known to be alive was taken from trial data; either date of last chemotherapy or date when came off study.

\section{Statistical analysis of the SIGNIFICANT trial}

The effect of treatment on overall survival time was investigated using Kaplan-Meier survival curves and Cox regression models. Univariable analysis included just treatment as a covariate within the model whilst multivariable analysis provided estimates of treatment effect adjusted for all relevant baseline prognostic factors that had been collected prospectively in the trial, specifically age $(<40$, 
40-59, 60+), World Health Organization (WHO) performance status (0-1,2+), cancer type (breast, small cell lung cancer, germ cell, non-Hodgkin's lymphoma, other) and chemotherapy setting

(adjuvant, non-adjuvant). It was recognized that any effect of levofloxacin on survival might depend upon patients' adherence to treatment. According to protocol, patients' adherence was recorded at the end of each cycle by counting the number of returned tablets and patients were categorised prospectively as overall adherers or not on the basis of taking at least $80 \%$ of the trial medication (a similar approach taken in other studies). The survival analysis was carried out separately for adherers and non-adherers. The analysis included all randomised patients for whom adherence data was available. In addition, in order to evaluate the effect of treatment on long-term survival, sensitivity analysis was carried out excluding patients who died during their planned chemotherapy treatment.

$(72)(73)(74)(74)(56)(56)(62)$

\section{Supplementary Figures}


A.

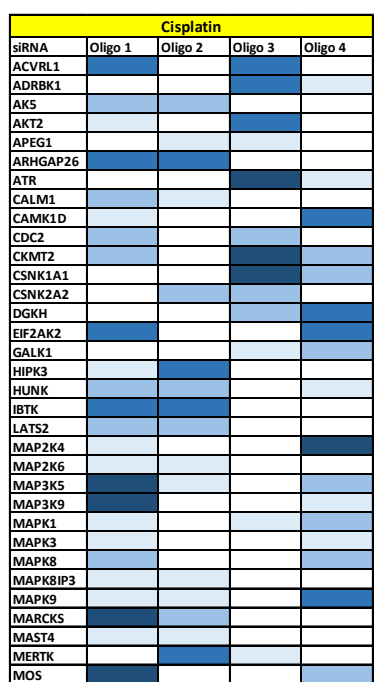

\begin{tabular}{|l|l|l|l|l|l|}
\hline colour & $>0.3$ & 0.2 to 0.3 & 0.15 to 0.2 & 0.1 to 0.15 \\
\hline Si
\end{tabular}

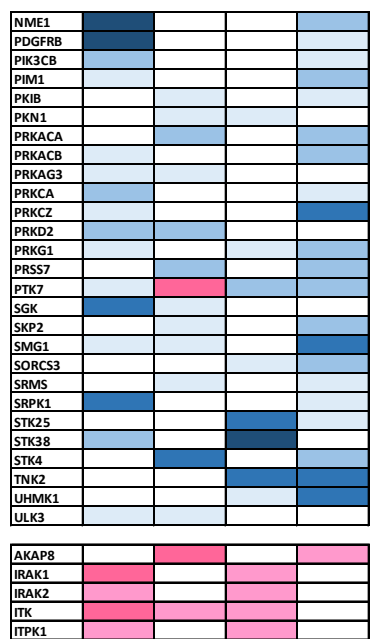

\begin{tabular}{|l|c|c|c|c|}
\hline Colour & $<-0.4$ & -0.3 to $-0.4-0.2$ to $-0.3-0.15$ to -0.2 \\
\hline Sl
\end{tabular}
B.

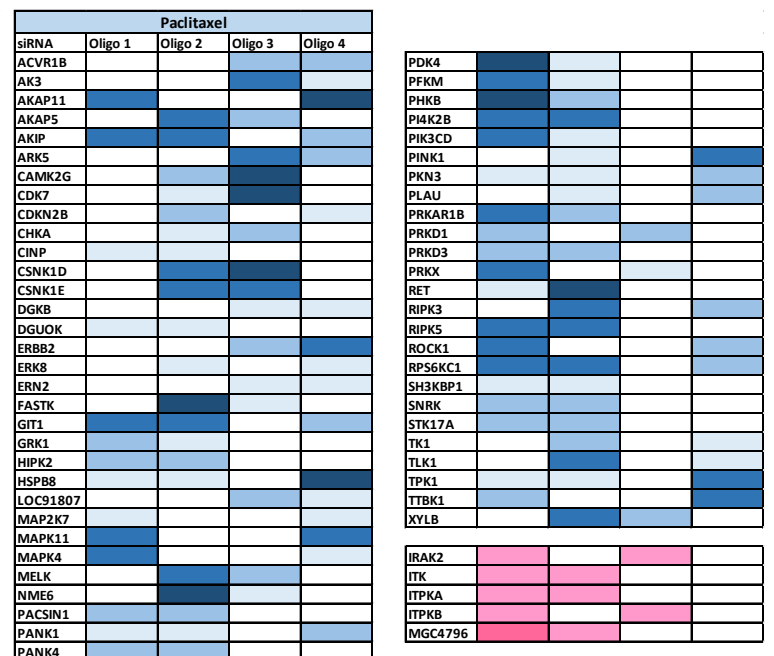

\begin{tabular}{|l|l|l|l|l|}
\hline colour & $>0.3$ & 0.2 to 0.3 & 0.15 to 0.2 & 0.1 to 0.15 \\
\hline Sl
\end{tabular}

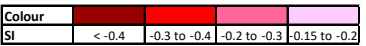

c.

\begin{tabular}{|c|c|c|c|c|c|c|c|c|}
\hline \multicolumn{4}{|c|}{ Cisplatin } & & \multicolumn{4}{|c|}{ Paclitaxel } \\
\hline Oligo 1 & Oligo 2 & Oligo 3 & Oligo 4 & siRNA & Oligo 1 & Oligo 2 & Oligo 3 & Oligo 4 \\
\hline & & & & ADK & & & & \\
\hline & & & & BMPR1A & & & & \\
\hline & & & & BMPR1B & & & & \\
\hline & & & & CDKL3 & & & & \\
\hline & & & & CKMT1 & & & & \\
\hline & & & & EEF2K & & & & \\
\hline & & & & EPHB6 & & & & \\
\hline & & & & GIT2 & & & & \\
\hline & & & & GNE & & & & \\
\hline & & & & IRAK2 & & & & \\
\hline & & & & $\mathrm{ITK}$ & & & & \\
\hline & & & & MAP4K4 & & & & \\
\hline & & & & MAPK10 & & & & \\
\hline & & & & $\begin{array}{l}\text { PDLIM5 } \\
\end{array}$ & & & & \\
\hline & & & & $\begin{array}{l}\text { PIP5K1C } \\
\end{array}$ & & & & \\
\hline & & & & PKN2 & & & & \\
\hline & & & & PRKAR2A & & & & \\
\hline & & & & PRKAR2B & & & & \\
\hline & & & & $\begin{array}{l}\text { PRKCB1 } \\
\end{array}$ & & & & \\
\hline & & & & RPS6KA6 & & & & \\
\hline & & & & SNF1LK2 & & & & \\
\hline & & & & STK22B & & & & \\
\hline & & & & TNNI3K & & & & \\
\hline & & & & ULK4 & & & & \\
\hline & & & & UMP-CMPK & & & & \\
\hline
\end{tabular}

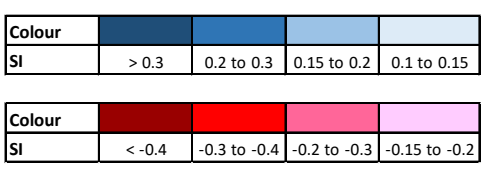

D.

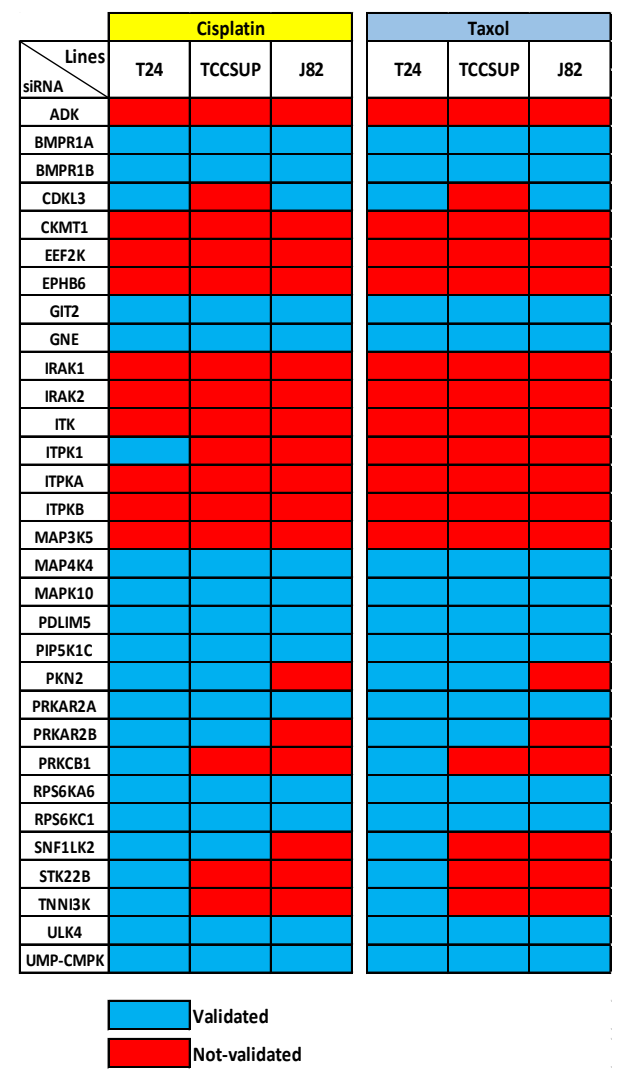

Fig S1: Kinome siRNA screen hits in T24 cells and validation results. (A-C) Heat maps of siRNA targets modulating the response of T24 cells to cisplatin only (A), taxol only (B) or both drugs (C) based on the sensitivity index (SI) (as defined in (48)). SI $>0.1$ highlights sensitisation whereas $\mathrm{SI}<0.15$ highlights resistance. Results for four individual targeting oligonucleotides per gene are shown. (D) Results of validation experiments performed in T24, TCCSUP and J82 bladder cancer cell lines. 

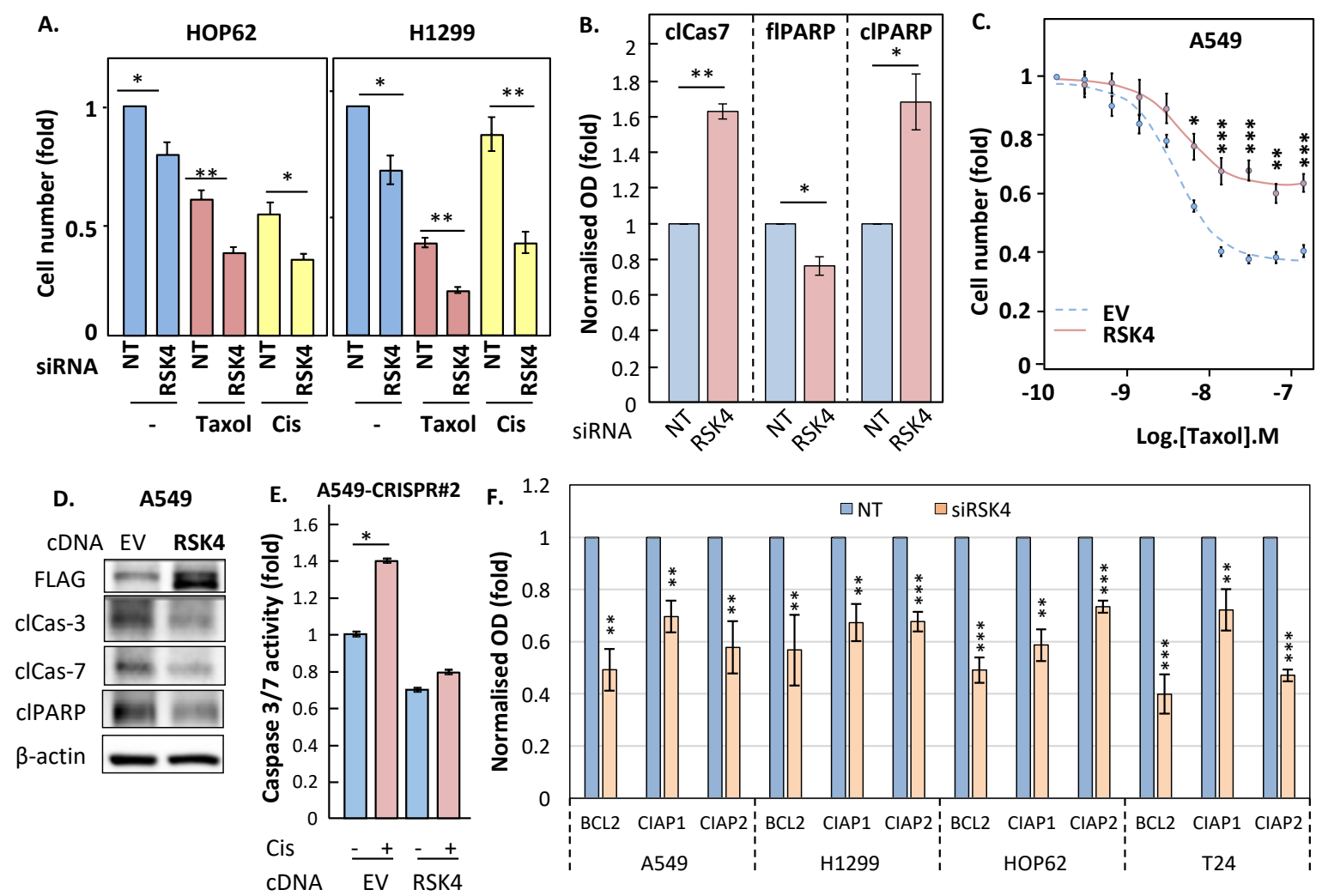

G.
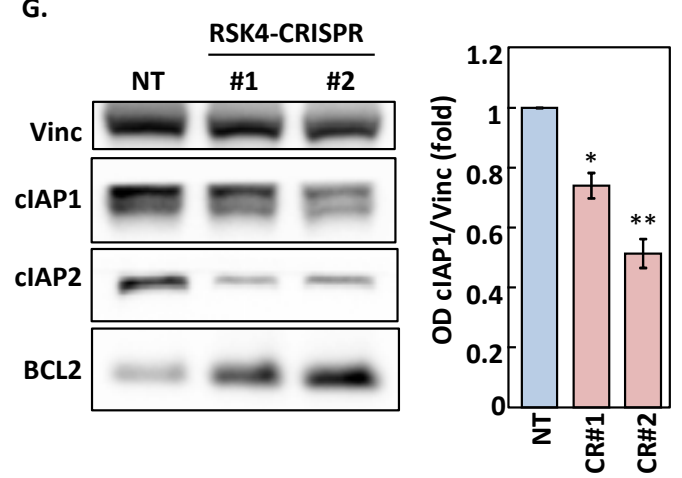

H.

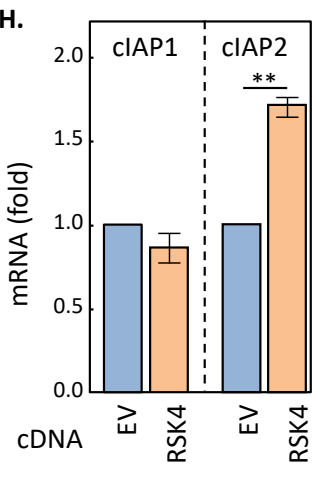

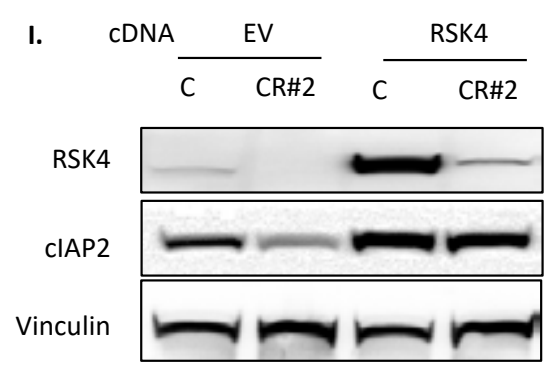
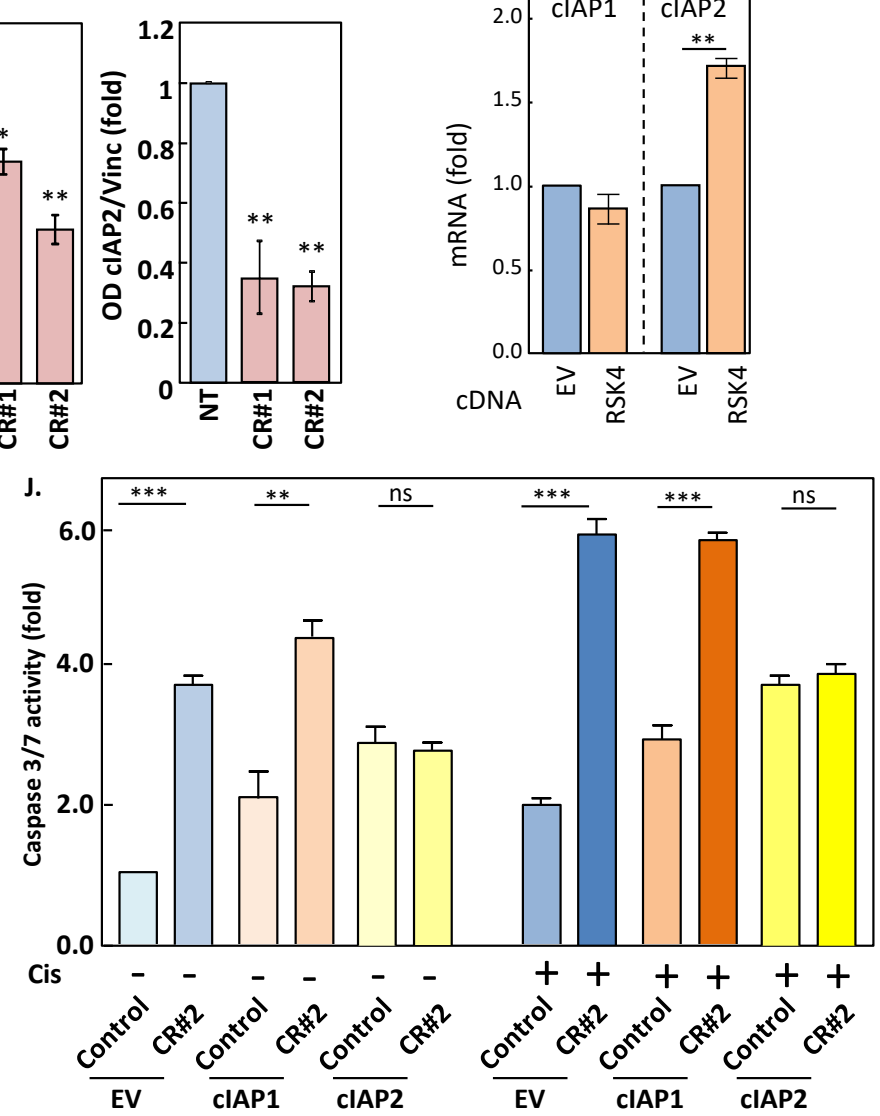

Fig S2: RSK4 modulates chemo-resistance in NSCLC cells. (A) siRNA-mediated silencing of RSK4 decreases cell survival to cisplatin $(12.5 \mu \mathrm{M})$ and taxol $(58 \mathrm{nM})$ in HOP62 and H1299 cells. NT; nontargeting. (B) Optical densitometry of Western blots for cleaved caspase 7, full-length and cleaved 
PARP normalised to vinculin expression from A549 cells treated with RSK4 or non-targeting (NT) siRNAs. (C) RSK4 overexpression in A549 renders them resistant to taxol. EV; empty-vector. (A-B) Cell number was determined by Crystal violet staining. Data shown are the mean \pm SEM of quadruplicates. (D and G) Lysates from A549 cells transfected with or without a RSK4-encoding or EV plasmid for $48 \mathrm{~h}$ (D) or A549 CRISPR non-targeting (NT) or RSK4 knockout (\#1, \#2) clones (G) were analysed by SDS-PAGE/Western blotting for the indicated proteins (left panel) and optical densitometry was performed on three biological replicates (right panels). Detection of $\beta$-actin or vinculin (Vinc) was used as a loading control. (A-D) Data shown are representative of at least biological triplicates. (E) A549 RSK4-CRISPR cells were transfected with empty vector (EV) or RSK4coding cDNA and caspase $3 / 7$ activity determined using a substrate-based assay. (F) Optical densitometry of Western blots for BCL2, CIAP1 and 2 normalised to vinculin expression from A549 cells treated with RSK4 or non-targeting (NT) siRNAs. (H) RT-qPCR for cIAP1 and 2 from A549 cells transfected with or without a RSK4-encoding or EV plasmid for $48 \mathrm{~h}$. (I) A549 NT and RSK4-CRISPR cells were transfected with EV or RSK4 CDNA and protein extracts were analysed by Western blotting for the indicated proteins $72 \mathrm{~h}$ later. (J) A549 control or RSK4-CRISPR cells were transfected with a ClAP1 or ClAP2-encoding or EV plasmid for $24 \mathrm{~h}$ prior to treatment \pm cisplatin for $48 \mathrm{~h}$ and caspase $3 / 7$ activity determination using a substrate-based assay. (C, E, F, G, H, I) Bar graphs represent mean \pm SEM of $\mathrm{n}=3$ or $4(\mathrm{H})$. Stats: Student's t-test with *; $p \leq 0.05,{ }^{* *} ; p \leq 0.01,{ }^{* * *} ; p \leq 0.001$, ns; nonsignificant. 

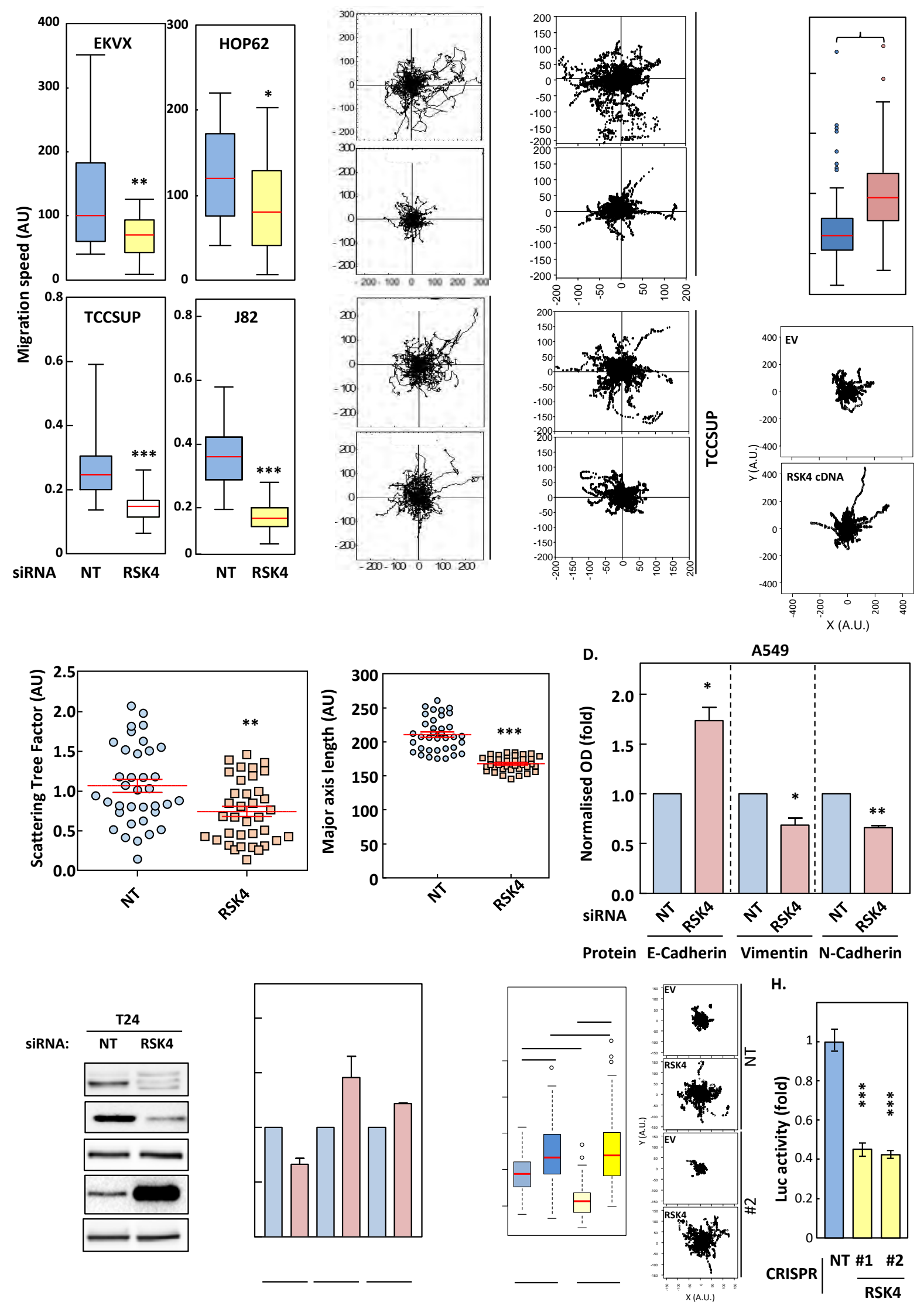

H.

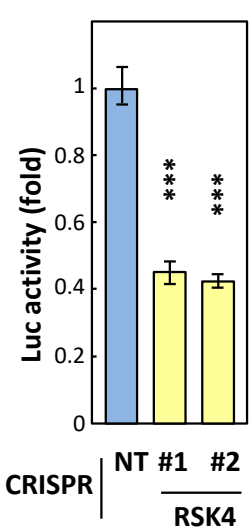

Fig S3: RSK4 modulates the metastatic program in lung and bladder cancer cells. (A-B) RSK4 silencing decreases (A) while RSK4 overexpression increases (B) the speed (A-left and B-up) and track 
length (A-right and B-bottom) of random-walk cell migration in lung (A549, EKVX and HOP62) and bladder (TCCSUP and J82) cancer cell lines. NT; non-targeting, EV; empty-vector. Red bar represent the median. (C) 36 microscopic fields of view for cells treated with non-targeting (NT) or RSK4targeting siRNAs such as those shown in Fig $2 \mathrm{C}$ were analysed in ImageJ to determine the Scattering Tree Factor or cellular Major Axis Length. Red bars represent mean \pm SEM. (D and F) Optical densitometry of Western blots $(n=3)$ for the indicated proteins normalised to vinculin expression from A549 cells transfected with RSK4 or non-targeting (NT) siRNAs (D) or empty-vector or RSK4encoding CDNA (F). (E) RSK4 silencing lead to a mesothelial-to-epithelial transition (MET) expression profile. Lysates from T24 cells treated for 72h with RSK4-targeting or NT siRNAs were analysed by SDS-PAGE/Western blotting for the indicated proteins. Detection of Vinculin was used as a loading control. E-Cadh; E-cadherin, N-Cadh; N-cadherin, Vim; vimentin, Vinc; vinculin. (F) Optical densitometry of Western blots $(n=3)$ for changes in EMT markers following RSK4 or empty-vector (EV) overexpression in A549 cells. Normalisation to vinculin signal was used as a loading control. (G) Overexpression of RSK4 rescues the decreased motility of RSK4-CRISPR A549 cells. NT or RSK4CRISPR cells (\#2) were transfected with either EV or RSK4 CDNA and random cell migration monitored using time-lapse microscopy for $18 \mathrm{~h}$. Left panel; Box-plot of cell speed. " $\mathrm{n}=$ "; number of cells tracked per condition. Right panel; individual cell tracks. (H) RSK4 or NT CRISPR A549 cells were transfected with a RelA-Firefly luciferase reporter construct and a Renilla luciferase control vector. The next day, lysates were analysed for Firefly luciferase activity normalised to that of the Renilla luciferase control using a luminescence plate reader. (A-D) Data shown are representative of three independent experiments with $(A, B, G)$ performed in quadruplicates. ( $D$ and $F$ ) Bar graphs represent mean \pm SEM of $n=3$. Statistics: (A-B and G) ANOVA and (C-F and H) t-test with *; $p \leq 0.05, * * ; p \leq 0.01$, $* * * ; p \leq 0.001$. 
A.
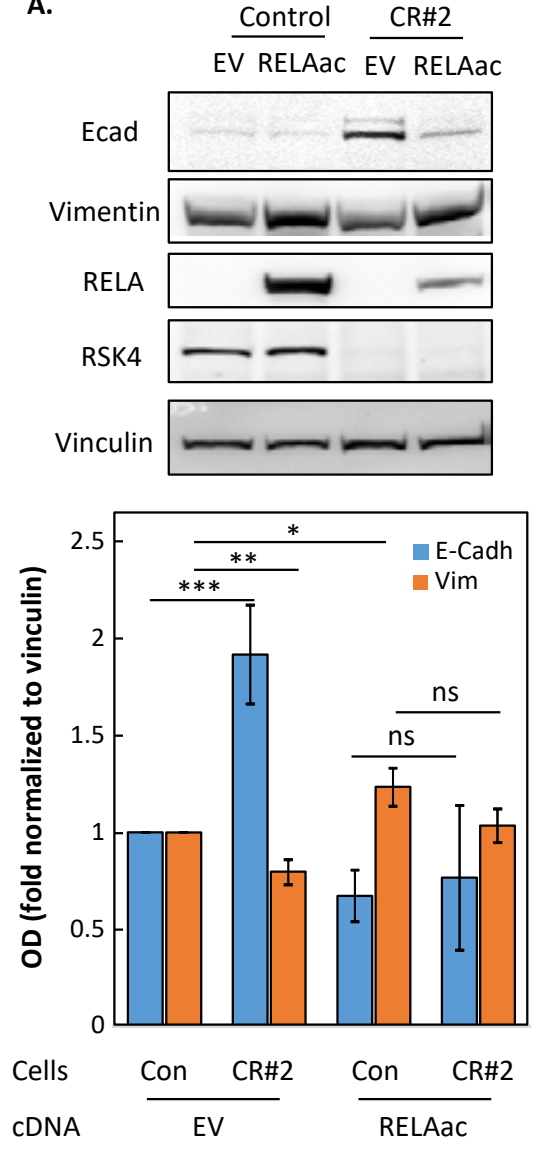

B.
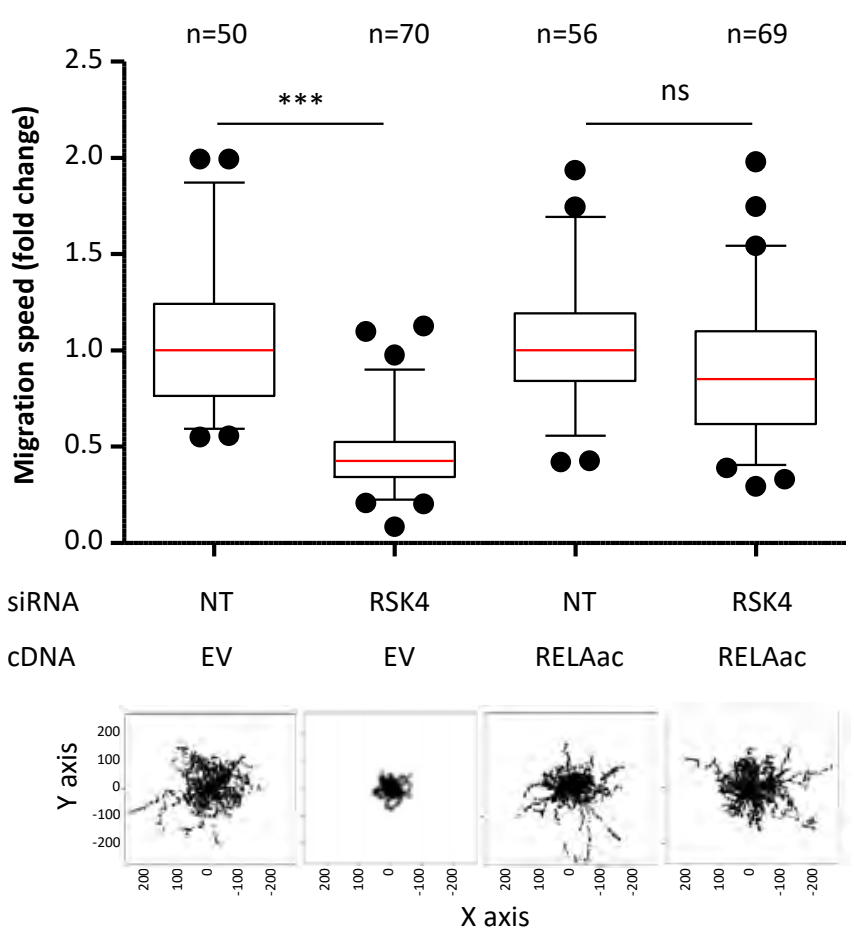

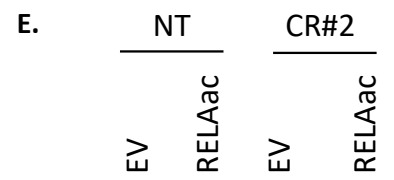

D.

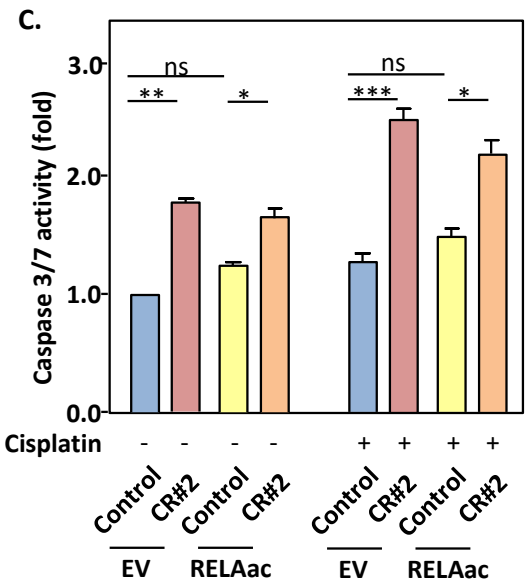

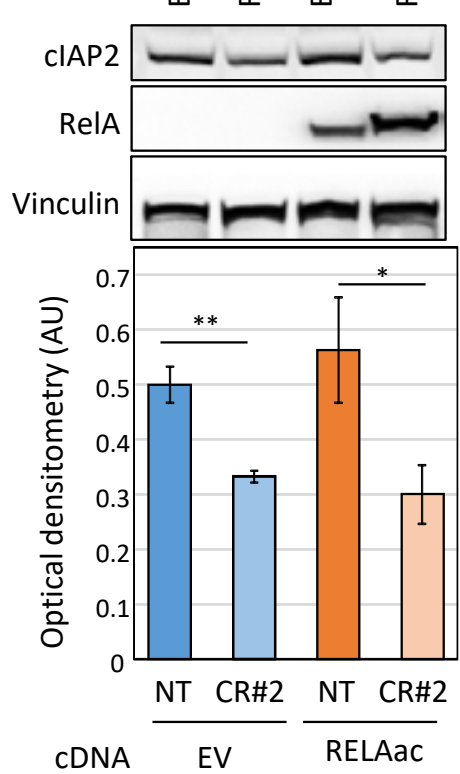

Fig S4: Active RELA overexpression rescues cell migration but not drug response changes downstream of RSK4 silencing. (A-B) Active RELA overexpression in RSK4-CRISPR (A) or wild-type A549 (B) corrects the changes in EMT markers (A) and the decrease in cell migration (B) observed following RSK4 downregulation. (A-lower panel) Optical densitometry quantification of $n=3$ repeats. CR\#2; RSK4 CRISPR clone 2, Con; control, EV; empty vector. (B) Cells were treated with nontargeting (NT) or RSK4-targeting siRNAs and transfected $24 \mathrm{~h}$ later with either empty vector or active RELA cDNA. Time-lapse imaging was performed $24 \mathrm{~h}$ later for $18 \mathrm{~h}$. Upper; Fold change in cell speed. 
Bottom; cell tracks. Red bar; median. Stats; ANOVA with ${ }^{* * *} ; p \leq 0.001$, ns; not significant. (C-E) Active RELA overexpression in RSK4-CRISPR (C and E) or wild-type A549 (D) does not prevent drug sensitisation downstream of RSK4 downregulation. (D) Cells treated with NT or RSK4-targeting siRNAs for $24 \mathrm{~h}$ or ( $\mathrm{C}$ and E) NT and RSK4-CRISPR cells were transfected with either empty-vector or active RELA cDNA. (C-D) $24 \mathrm{~h}$ later, cells were treated with or without cisplatin for $48 \mathrm{~h}$ and caspase $3 / 7$ activity determined using a substrate-based luminometric assay. (E) $72 \mathrm{~h}$ after vector transfection, cell lysates were analysed by Western blotting for the indicated proteins (upper panel) and repeats $(n=3)$ were quantified by optical densitometry for changes in clAP2 expression, normalised to those of vinculin used as a loading control (lower panel). Bar graphs represent mean \pm SEM. Stats; Student's t-test with *; $p \leq 0.05,{ }^{* *} ; p \leq 0.01,{ }^{* * *} ; \leq \leq 0.001, \mathrm{~ns}$; not significant. 

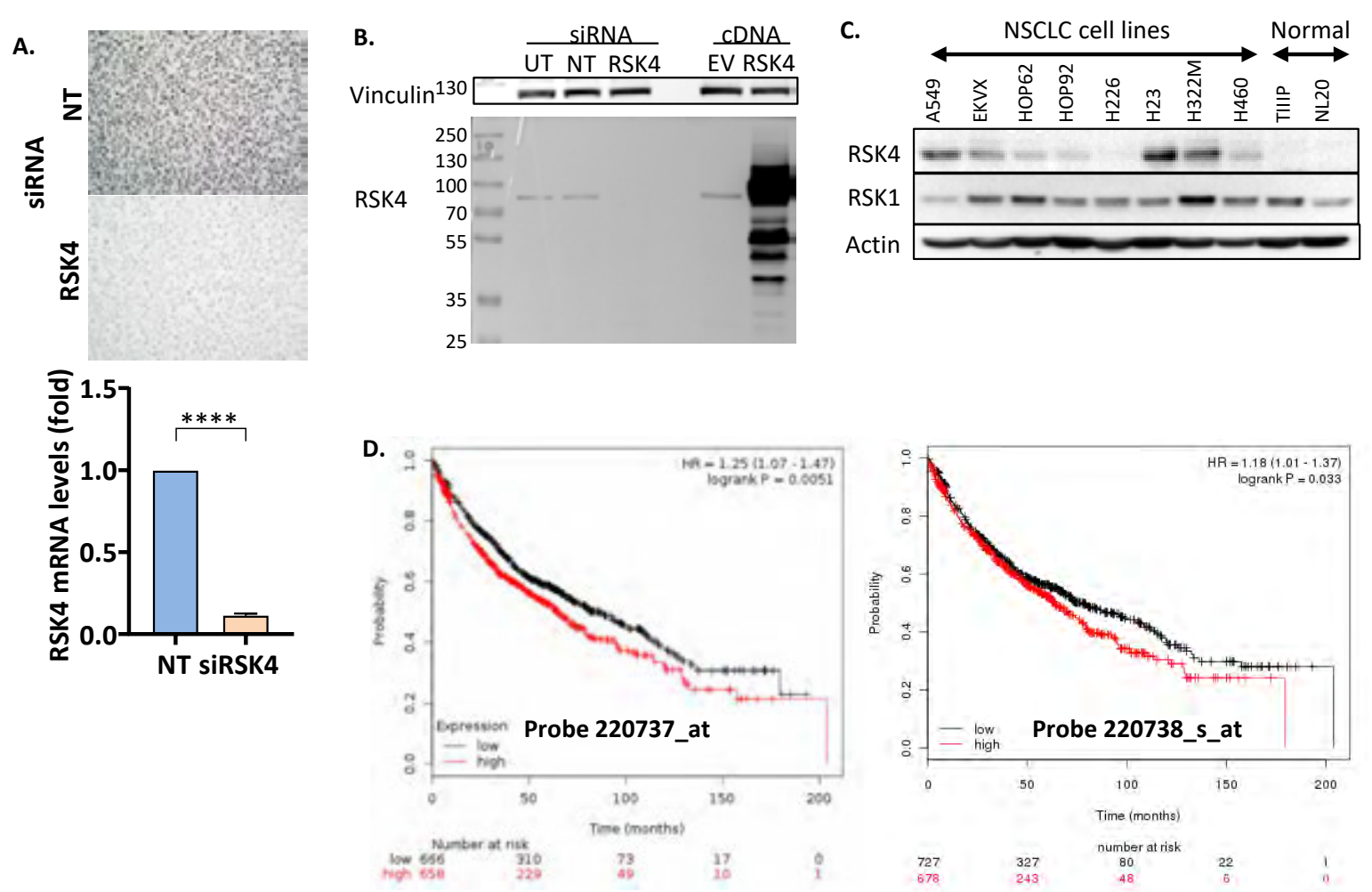

E.

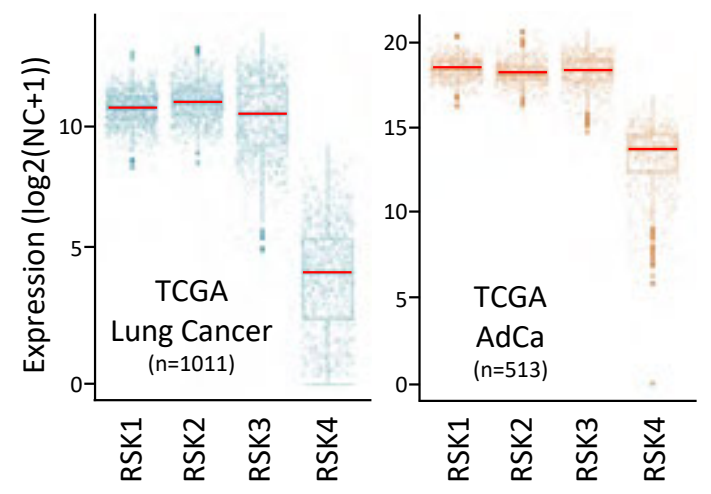

F.

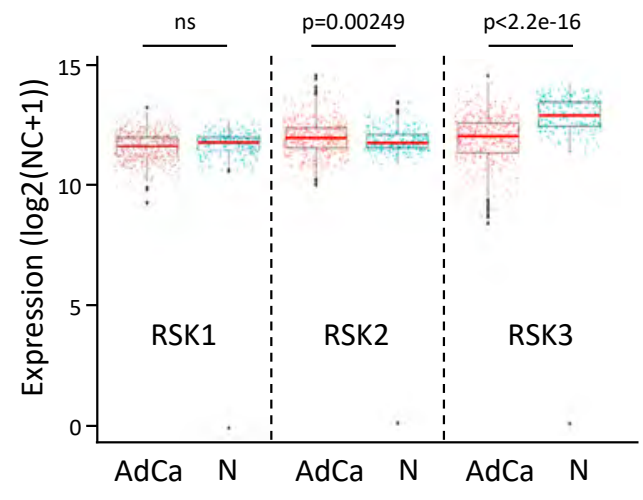

G.
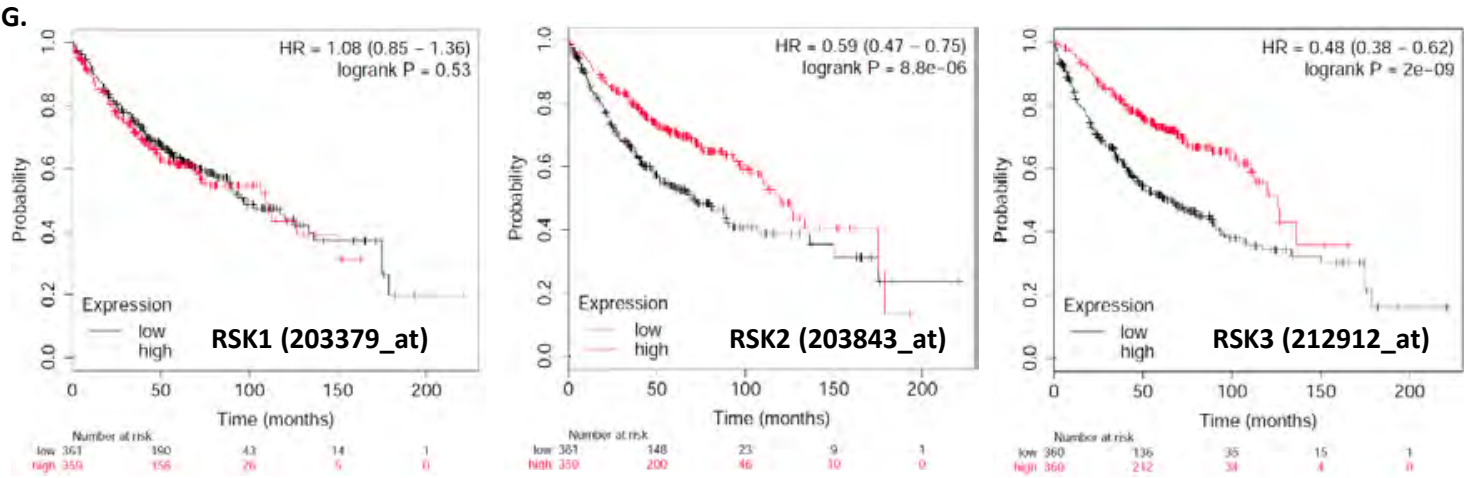

Fig S5: RSK4 overexpression in lung cancer cells and clinical relevance. (A) A549 cells were transfected with RSK4 or non-targeting (NT) siRNAs for $48 \mathrm{~h}$ prior to centrifugation, fixation and paraffin embedding. Slices were stained for RSK4 expression and counterstained by DAB. Lower panel: corresponding representative RT-qPCR results. Data are average of 3 technical replicates \pm SD. 
(B) Lysates from A549 cells transfected or not (UT) for $48 \mathrm{~h}$ with non-targeting (NT) or RSK4 siRNAs or empty-vector or RSK4 cDNA were analysed by Western blotting to demonstrate the antibody's specificity. Full blot is shown. Vinculin detection was used as a loading control. (C) Lysates from the indicated cell lines were analysed by SDS-PAGE/Western blotting for the indicated proteins with actin serving as a loading control. PIIG; type-II pneumocytes. (D) Kaplan-Meier analysis of overall survival for lung cancer patients (all subtypes) as a function of low or high RSK4 mRNA expression. Analysis was performed using the Km-plotter web interface (75) using two separate microarray probes. Median mRNA expression was used to determine the cut-off between low and high expressors. (E-F) The TCGA lung cancer datasets was analysed for respective expression of RSK isoforms in cancer samples (E) or for comparison of expression of RSK1-3 between normal lung (N) and lung adenocarcinoma (AdCa) samples (F). Stats: Student's t-test. (G) Kaplan-Meier analysis of overall survival for lung adenocarcinoma patients as a function of low or high mRNA expression for $R S K 1, R S K 2$, and RSK3. Analysis was performed using the Km-plotter web interface (75) using the indicated microarray probes. Median mRNA expression was used to determine the cut-off between low and high expressors. 


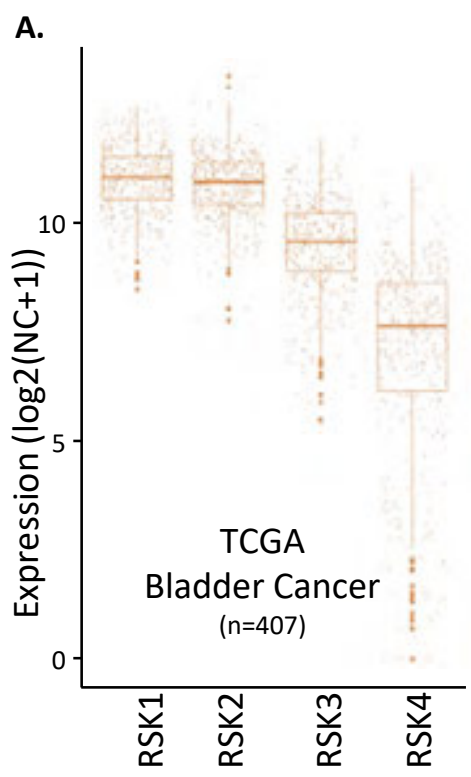

B.
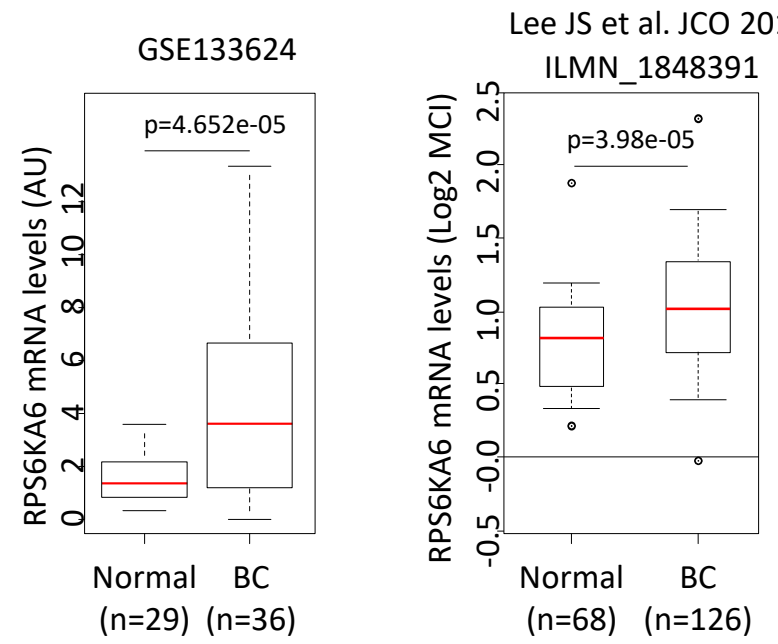

Sanchez-Carbayo M et al. JCO 2006

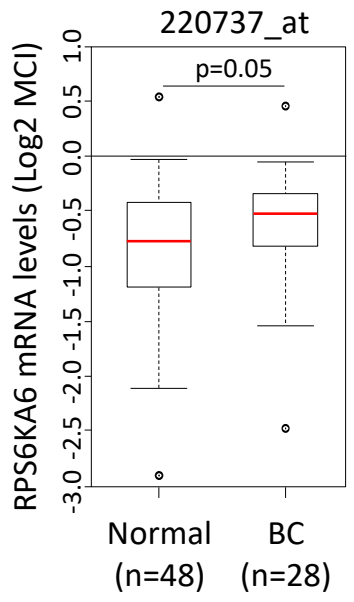

Fig S6: RSK4 is overexpressed in bladder cancer. (A) The bladder cancer TCGA dataset was analysed for the relative expression of RSK isoforms. Red bar; median. (B) Two microarray and one RNA-Seq (GSE133624) datasets were analysed for comparative expression of RSK4 between cancer (BC) and normal tissue. For the microarray datasets, the probes used to detect RSK4 mRNA are indicated. Red bar; median. Stats: Student's t-test.

\begin{tabular}{|c|c|c|c|c|c|c|c|}
\hline & Mouse & $\begin{array}{c}\text { Av lung } \\
\text { involvement }\end{array}$ & $\begin{array}{c}\text { Av } \\
\text { diameter } \\
(\mathrm{mm})\end{array}$ & & Mouse & $\begin{array}{c}\text { Av lung } \\
\text { involvement }\end{array}$ & $\begin{array}{c}\text { Av } \\
\text { diameter } \\
(\mathrm{mm})\end{array}$ \\
\hline \multirow{6}{*}{$\begin{array}{l}\frac{\mathscr{U}}{\varepsilon} \\
\frac{5}{z}\end{array}$} & $\# 1$ & 70 & 0.15 & \multirow{6}{*}{ 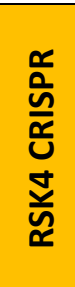 } & $\# 1$ & 30 & 0.064 \\
\hline & $\# 2$ & 60 & 0.25 & & $\# 2$ & 3 & 0.04 \\
\hline & $\# 3$ & 80 & 0.75 & & $\# 3$ & 1 & 0.016 \\
\hline & $\# 4$ & 30 & 0.066 & & \#4 & 2 & 0.012 \\
\hline & \#5 & 0 & 0 & & \#5 & 40 & 0.134 \\
\hline & $\# 6$ & 60 & 0.27 & & \#6 & 2 & 0.013 \\
\hline
\end{tabular}




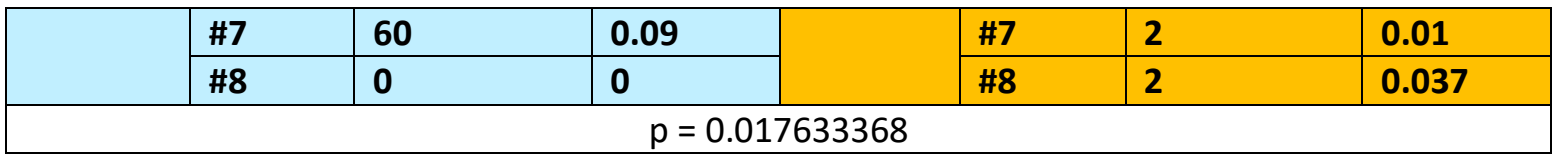

Fig S7: RSK4 downregulation prevents lung colonisation by A549 cells. $1.10^{6}$ A459 NT or RSK4 CRISPR cell clone \#1 were injected through the tail vein of SCID mice. Animals were sacrificed 2 months later and their lungs extracted, fixed and paraffin-embedded prior to 10 sections (each separated by $60 \mu \mathrm{m}$ ) being $\mathrm{H} \& \mathrm{E}$ stained and the average lung involvement (\%) and average diameter of lung tumour nodules determined. 


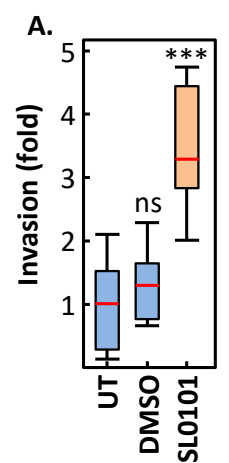

D.

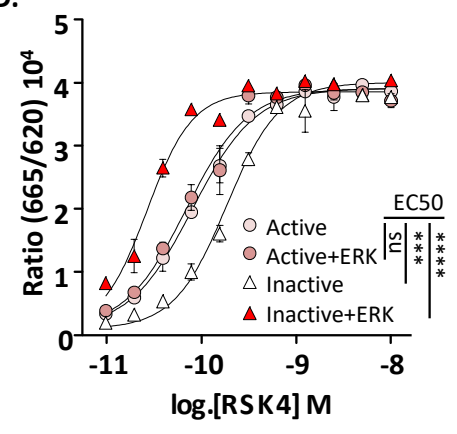

G.

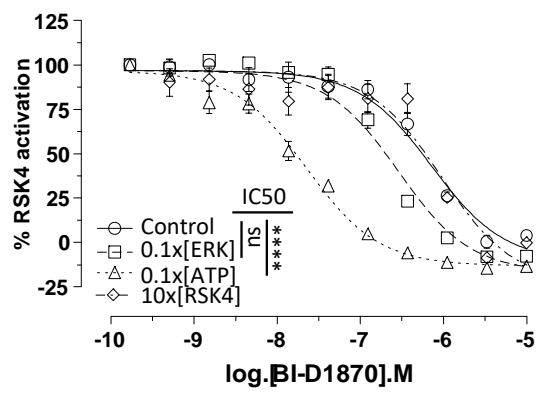

I.

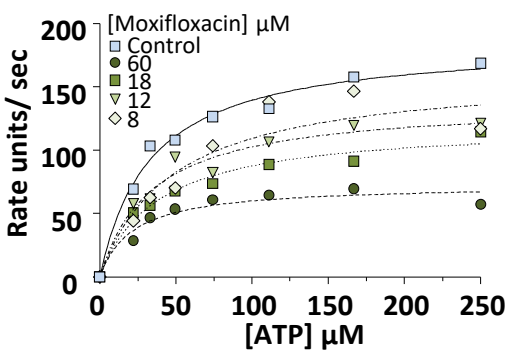

K.

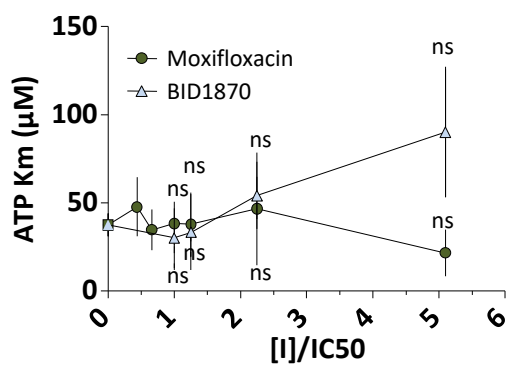

E. c.

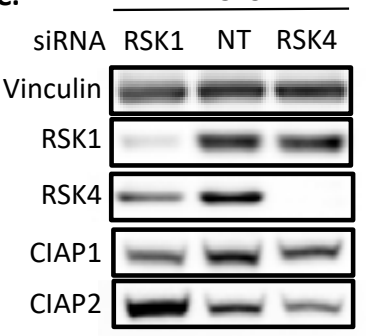

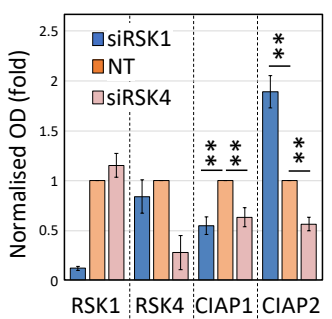
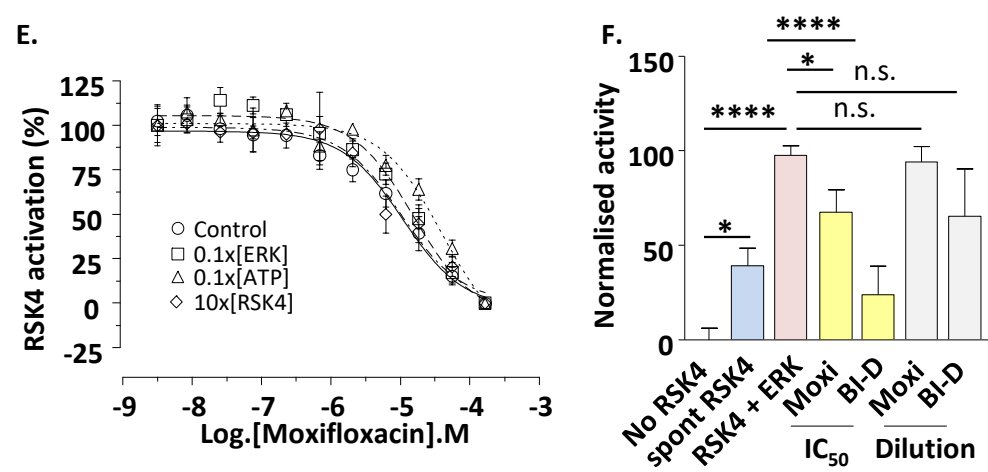

H.

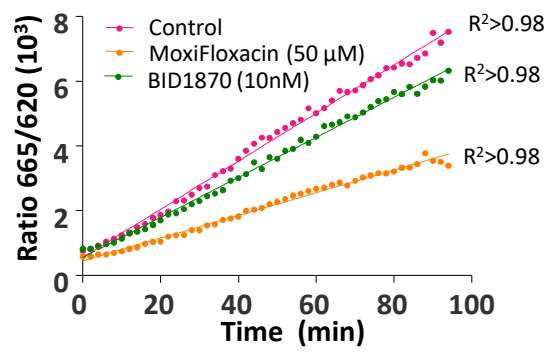

J.

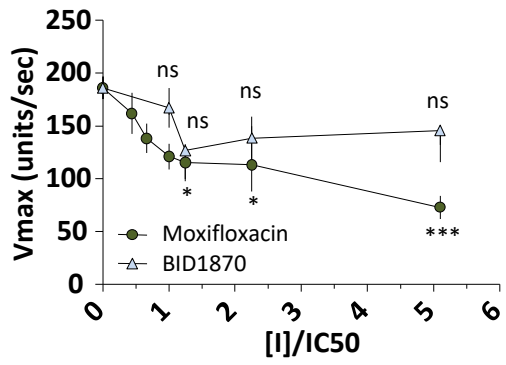

L.

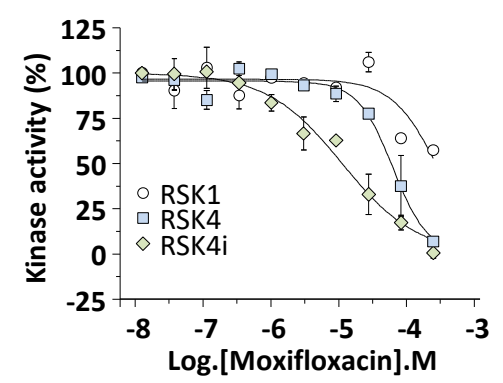

Fig S8: Rational for and identification of a RSK4 allosteric inhibitor. (A-C) RSK1 inhibition produces opposite effects on cell invasion and drug sensitisation as RSK4 downregulation. (A) A549 were 
treated with or without DMSO or $50 \mu \mathrm{M}$ of the pan-RSK inhibitor SL0101 prior to being overlaid with a collagen I matrix and $166 \mathrm{ng} / \mathrm{ml}$ EGF to provide chemo-attraction. Cell invasion was monitored $48 \mathrm{~h}$ later by confocal microscopy following PFA-based fixation and Cytox Green staining. Box plot is representative of three independent experiment performed in quadruplicates with a total of 36 fields of view acquired. Red bar represents the median. (B) Comparison of changes in sensitivity index to Cisplatin and Taxol obtained following silencing of RSK4 or RSK1 in lung (A549) or bladder (T24, TCCSUP and J82) cells lines. Cells were transfected with the indicated siRNAs or a non-targeting sequence and treated two days later with cisplatin ( $3.5 \mu \mathrm{M}$ for bladder cells and $12.5 \mu \mathrm{M}$ for A549 cells) and taxol ( $40 \mathrm{nM}$ for bladder cells and $58 \mathrm{nM}$ for A549 cells) for $48 \mathrm{~h}$. Cell number was determined by Crystal violet staining and the sensitivity index calculated as in (48). Statistics: ANNOVA with n.s.; not significant, *; $p<0.05,{ }^{* *} ; p<0.01,{ }^{* * *} ; p<0.001$. (C) Left: Lysates for A549 cells treated with NT, RSK1 or 4 siRNAs were analysed by Western blotting for the indicated proteins. Vinculin detection was used as a loading control. Right: Optical densitometry of three biological replicate experiments normalised to vinculin and to the NT condition. (D) Dephosphorylated "inactive" RSK4 can be efficiently re-activated in vitro in the presence of recombinant active ERK2 as monitored using an $\mathrm{HTRF}^{\circledR} \mathrm{KinEASE}^{\mathrm{TM}}$ platform. (E) Varying the concentration of ATP, ERK2 or RSK4 in the assay does not modify the inhibitory efficiency of moxifloxacin. (F) Dilution of pre-inhibited RSK4 using $\mathrm{IC}_{50}$ for the indicated compounds rescues kinase activity, suggesting resversibility of the inhibition. BI-D; BI-D1870, Moxi; moxifloxacin. (G) HTRF-based RSK4 activation assays in the presence of the pan-RSK inhibitor, BI-D1870. Varying the concentration of ATP and ERK2, but not RSK4, modifies the inhibitory efficiency of BI-D1870. (H) Time-dependence assays for inhibition by moxifloxacin and BI-D1870 show linear relationship with product formation in the presence of both inhibitors. (I) Michaelis Menten kinetics for RSK4 in the presence of increasing concentrations of moxifloxacin show reduced Vmax (J) but maintenance of Km (K). (L) Moxifloxacin demonstrates higher potency against the dephosphorylated RSK4 (RSK4i) than activated RSK1 or 4. Moxifloxacine was incubated with dephosphorylated RSK4 (RSK4i), active/phosphorylated RSK4 (RSK4) or RSK1 and the enzymes subjected to HTRF assays in the presence of ERK2. (A-L) Results shown are representative of at least three independent repeats. (B-K) Data represent the mean of $n=4$ replicates \pm SEM. (A, B and E) Statistics: Student's t-test with n.s.; not significant, ${ }^{*} ; p \leq 0.05,{ }^{* *}$; $p \leq 0.01, * * * ; p \leq 0.001$. 
A.
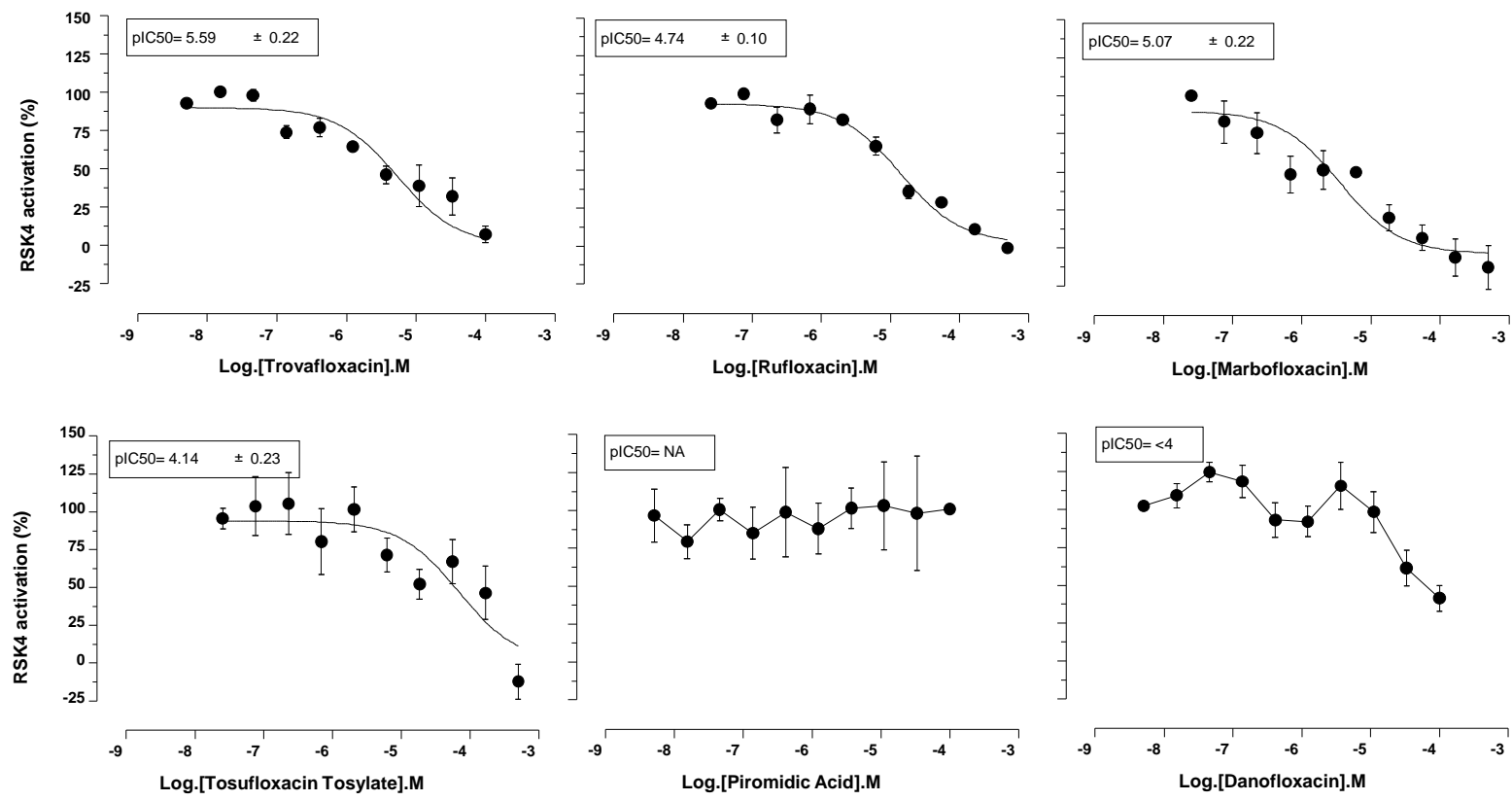

Fig S9: Not all floxacins inhibit RSK4 activation. (A) HTRF-based RSK4 activation assays in the absence or presence of increasing concentrations of the indicated representative floxacin compounds show that these vary in their ability to inhibit RSK4 activation by ERK2. 


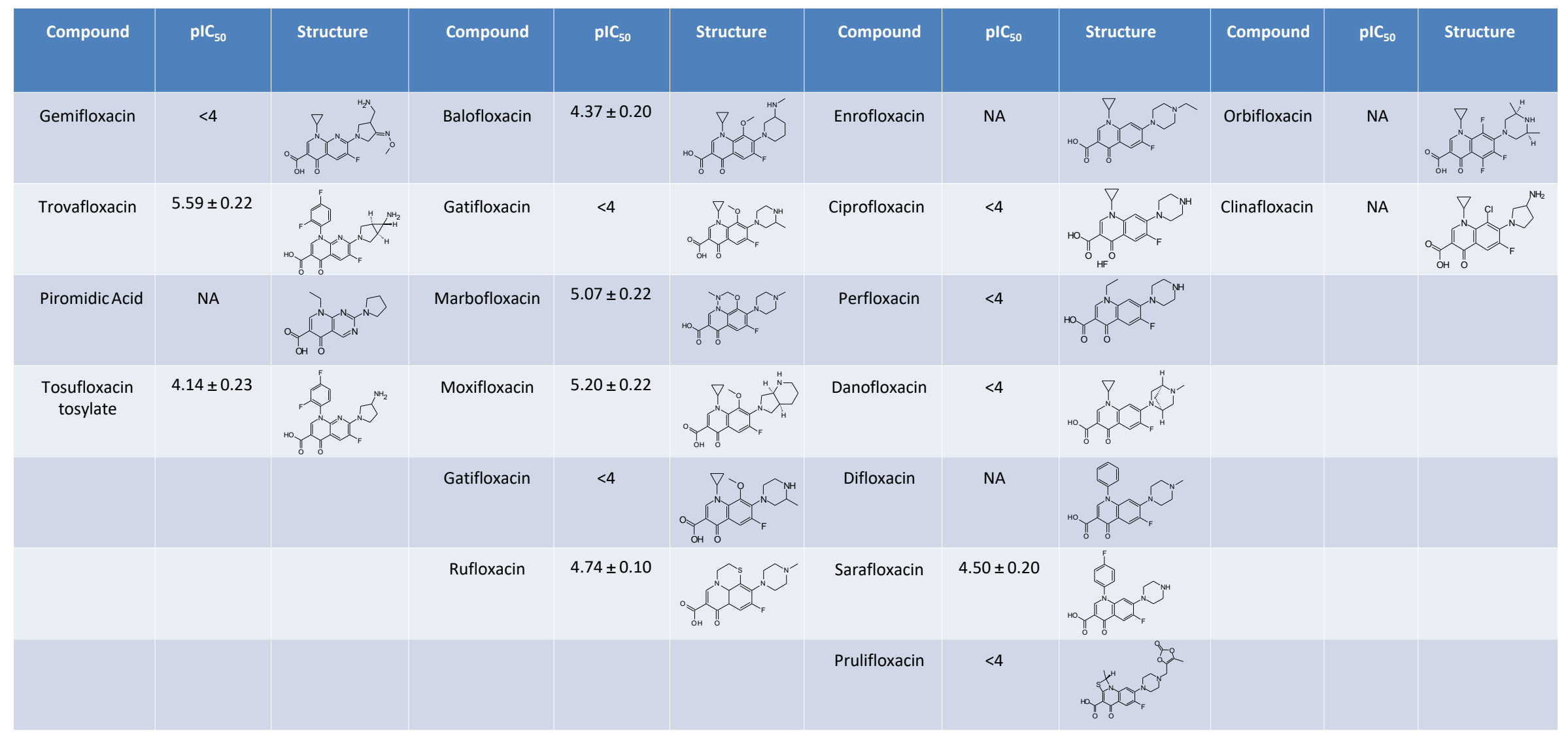

Fig S10: Floxacin compounds tested in our RSK4 activation assay, corresponding structures and pIC 50 values. NA (Not Applicable) values points to compounds that failed to inhibit RSK4 activation at all tested concentrations. 

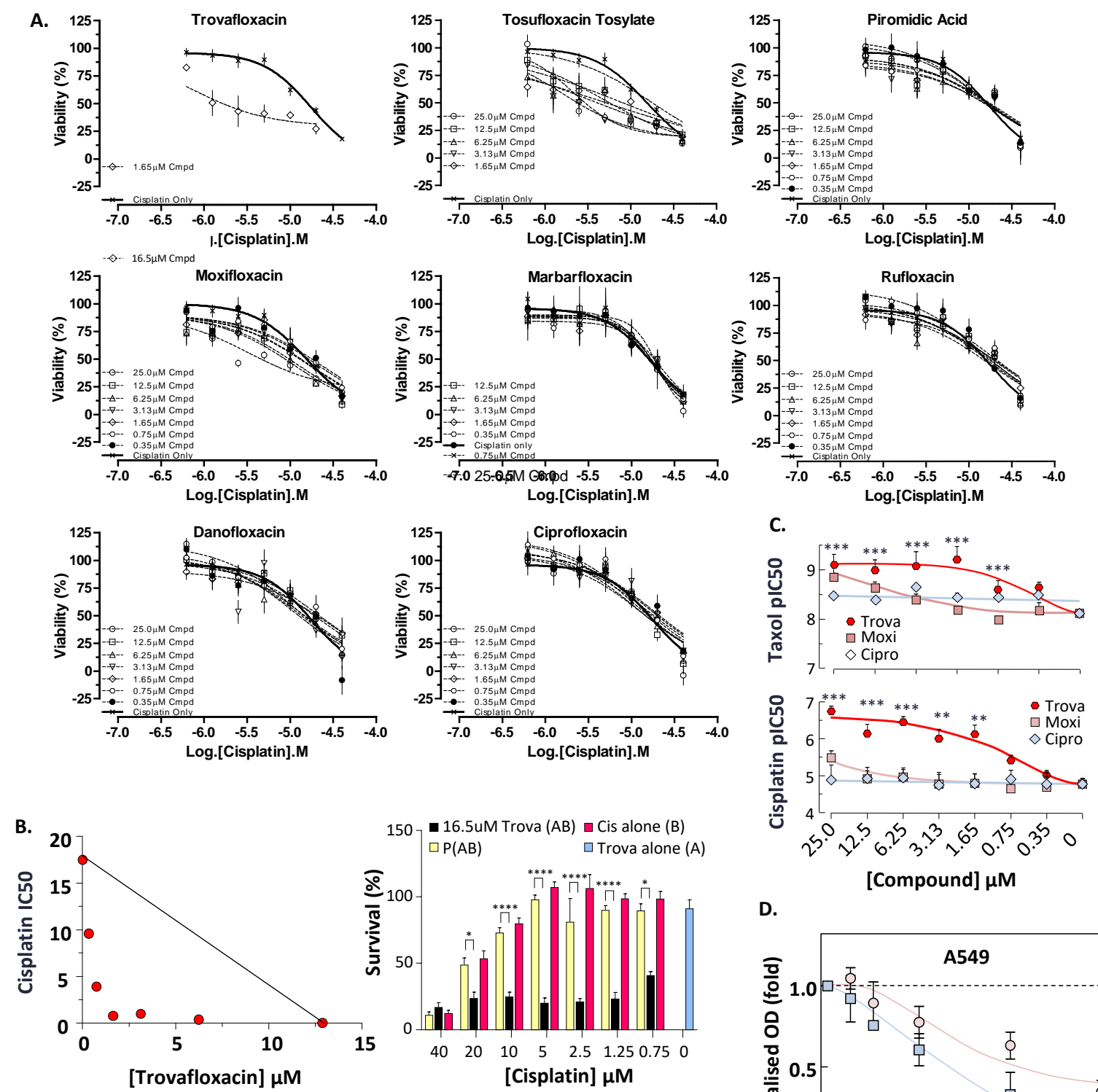

D.

E.

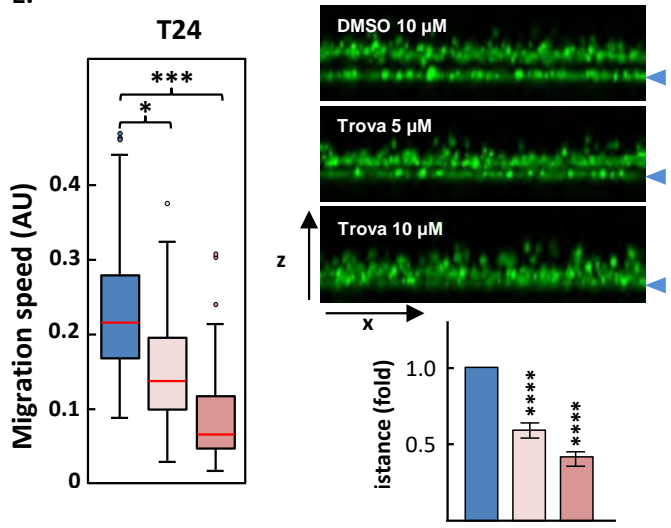

G. Trova

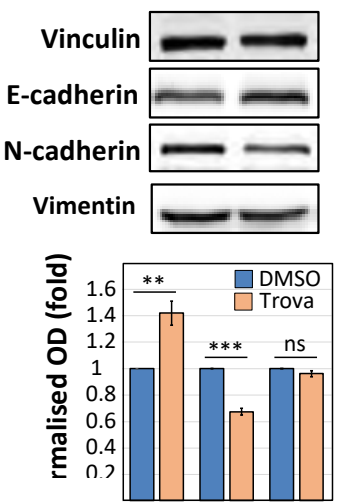

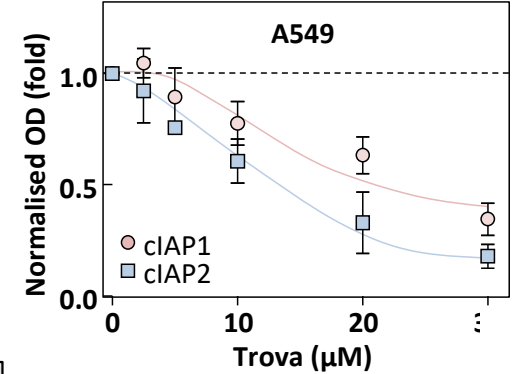

H.

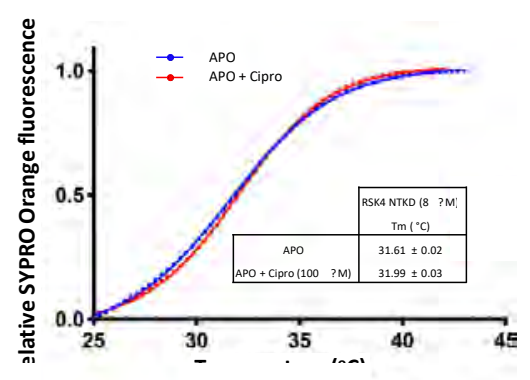

Fig. S11: Floxacins inhibiting RSK4 reproduce cellular effects of RSK4 knockdown. (A) Floxacin compounds differ in their ability to sensitise A549 cells to cisplatin. Cells were treated with various 
concentrations of floxacins for $1 \mathrm{~h}$ prior to incubation with a dose range of cisplatin for $72 \mathrm{~h}$ before determining cell number by Alamar Blue staining. (B) Left: Isobologram for Cisplatin $\mathrm{IC}_{50}$ versus a concentration range for trovafloxacin demonstrates synergism between the two compounds in A549 cell viability assays. Right: Synergism is separately confirmed by corresponding Bliss independence factors (P(AB)) calculation. Statistics 2-way ANOVA with *; $p<0.05,{ }^{* *} ; p \leq 0.01,{ }^{* * *} ; p<0.005,{ }^{* * * *}$; $p \leq 0.001$. (C) Trovafloxacin shows increased ability over moxifloxacin in sensitising A549 cells to cisplatin and taxol. Cells were incubated with floxacin compounds for $1 \mathrm{~h}$ prior to addition of taxol or cisplatin. Cell viability was determined 48h later using Alamar Blue. (D) Optical densitometry of Western blots $(n=3)$ for cIAP1 and 2 of lysates from A549 cells treated with a dose range of trovafloxacin. Values are normalised to vinculin expression and to the untreated condition. (E-F) T24 $(E)$ and A549 (F) cells were subjected to (E) a random-walk cell migration assay for $18 \mathrm{~h}$ or (F) a 3Dcollagen invasion assay following treatment with or without trovafloxacin at the indicated concentrations. (E) $\mathrm{N}=80$ cells were tracked per condition. Red bar; median. (F) Upper; representative confocal stacks with blue arrow representing bottom of plate. Lower; bar graph represent mean \pm SEM of 9 stacks. (G) Cell lysates from A549 cells treated with/without $10 \mu \mathrm{M}$ trovafloxacin for $24 \mathrm{~h}$ were analysed by Western blotting for the indicated proteins. Detection of vinculin was used as a loading control. Lower panel shows quantification of three biological replicates. (H) Differential Scanning Fluorimetry analysis of Ciprofloxacin (negative control) binding to RSK4 NTKD. Shown are the melting curves of dephosphorylated RSK4 NTKD (blue) and RSK4 NTKD in presence of $100 \mu \mathrm{M}$ Ciprofloxacin (red). Ciprofloxacin did not induce any notable temperature shifts indicating no binding to RSK4 NTKD. To maximise solubility, Ciprofloxacin was dissolved in $\mathrm{HCl}$ and the $\mathrm{HCl}$ final concentration in both RSK4 NTKD APO and Ciprofloxacin samples was $0.7 \mu \mathrm{M}$. (A-G) Results shown are representative of at least three independent experiments. Statistics: ANOVA with *; $p<0.05, * * ; p \leq 0.01,{ }^{* * *} ; p<0.005, * * * * ; p \leq 0.001$. 
A.

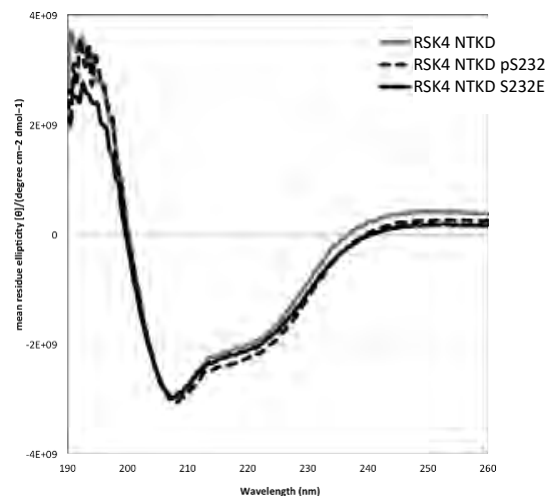

B.

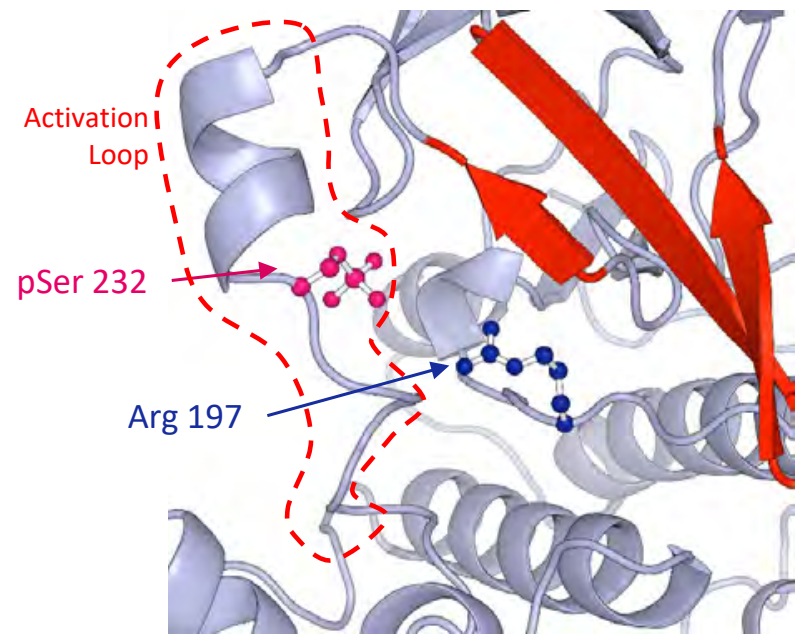

D.

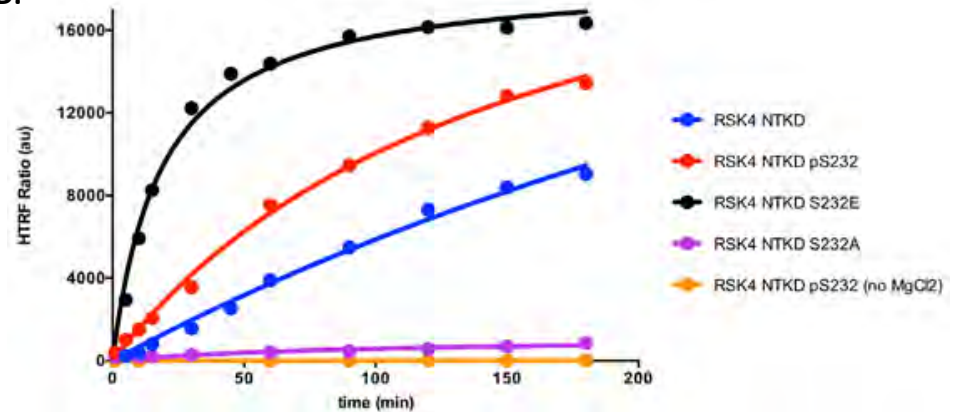

C.

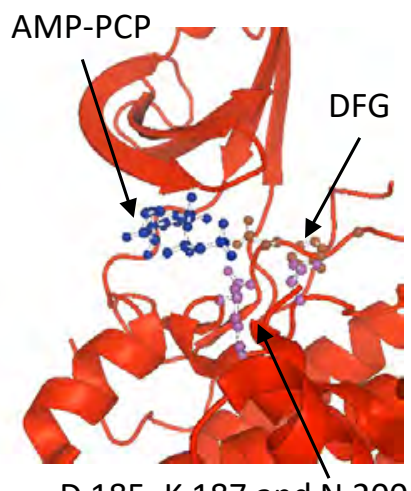

D 185, K 187 and N 200

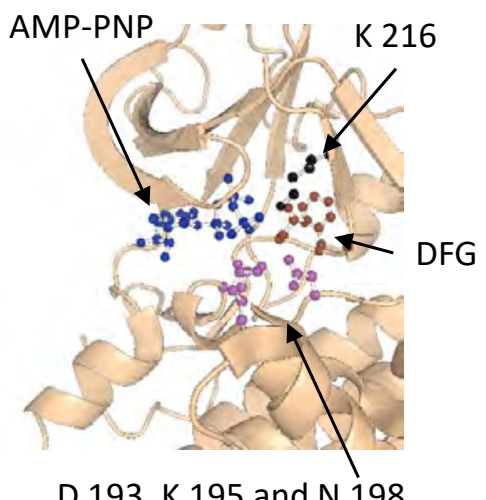

D 193, K 195 and N 198

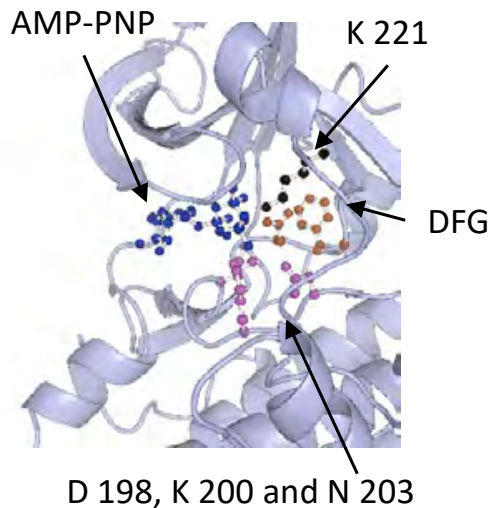

Fig. S12: RSK4 structures and floxacin docking. (A) Far UV CD spectrum of RSK4 NTKD, RSK4 NTKD pS232 and RSK4 NTKD S232E reported in terms of mean residue ellipticity $[\theta] /\left(\right.$ degree $\left.\cdot \mathrm{cm}^{-2} \cdot \mathrm{dmol}^{-1}\right)$. Deconvolution of the CD spectra of RSK4 NTKD, RSK4 NTKD pS232 and RSK4 NTKD S232E yield, respectively, the following percentages for secondary structure elements: $21.1 \%$ of $\alpha$-helix, $31.6 \%$ of $\beta$-strands and $17.6 \%$ turns; $22.7 \%$ of $\alpha$-helix, $29.3 \%$ of $\beta$-strands and $20.9 \%$ turns; $18.8 \%$ of $\alpha$-helix, $29.7 \%$ of $\beta$-strands and $20.8 \%$ turns. All the variations are within the uncertainty of the techniques and show no significant differences in the composition of secondary structural elements among the different proteins. (B) Cartoon representation of RSK4 NTKD pS232. $\beta$ B-sheet is highlighted in red. In ball-and-stick are shown the phosphorylated S232 (pink) and R197, part of the HDR motif (blue). (C) Top panel: RSK1 NTKD active site bound to AMP-PCP (the AMP-PCP is in an orientation not favourable for $\psi$-phosphate transfer). Middle panel: RSK2 NTKD active site bound to AMP-PNP and Lower panel: RSK4 active site bound to AMP-PNP. In ball-and-stick are shown key residues of the 
active site: the DFG motif, which positions ATP for phosphoryl transfer, is shown in brown; the substrate binding $D$, the catalytic $K$ (indispensable for the formation of enzymatic intermediate during catalysis ) and the magnesium-binding $\mathrm{N}$, located at the bottom of the active sites, are shown in purple; the $\mathrm{K}$, which interacts with the $\beta$-phosphate of the ATP and help the correct positioning required for ATP binding and catalysis, is shown in black (part of the $\beta B$-sheet motif which is not visible in RSK1 structure). The relative position of the key residues reveals an almost identical arrangement between RSK1/2/4 NTKD active sites. All structures have been superimposed and are shown in the same orientation. (D) Kinase activity of RSK4 mutants. Hetero-phosphorylation of the HTRF biotinylated peptide S2 $(1 \mu \mathrm{M})$ by $500 \mathrm{nM}$ of wild-type or indicated mutant versions of RSK4 NTKD in the presence of $10 \mathrm{mM} \mathrm{MgCl} 2$ and $2 \mathrm{mM}$ ATP. The time points follow a classic MichaelisMenten trend, showing that our recombinant RSK4 proteins are fully functional in vitro, independent of PDK1 activity. DSF and HTRF experiments were run using the same buffer used to crystallise the RSK4 NTKD, RSK4 NTKD pS232 and RSK4 NTKD S232E. (E-F) Docking Controls. Cartoon representation of RSK4 bound to Trovafloxacin and two positive and negative controls. All structures are in the same orientation and were superimposed using the RSK4 NTKD structure. The $\beta B$-sheet is highlighted in red and the activation loop in pink. 

A.

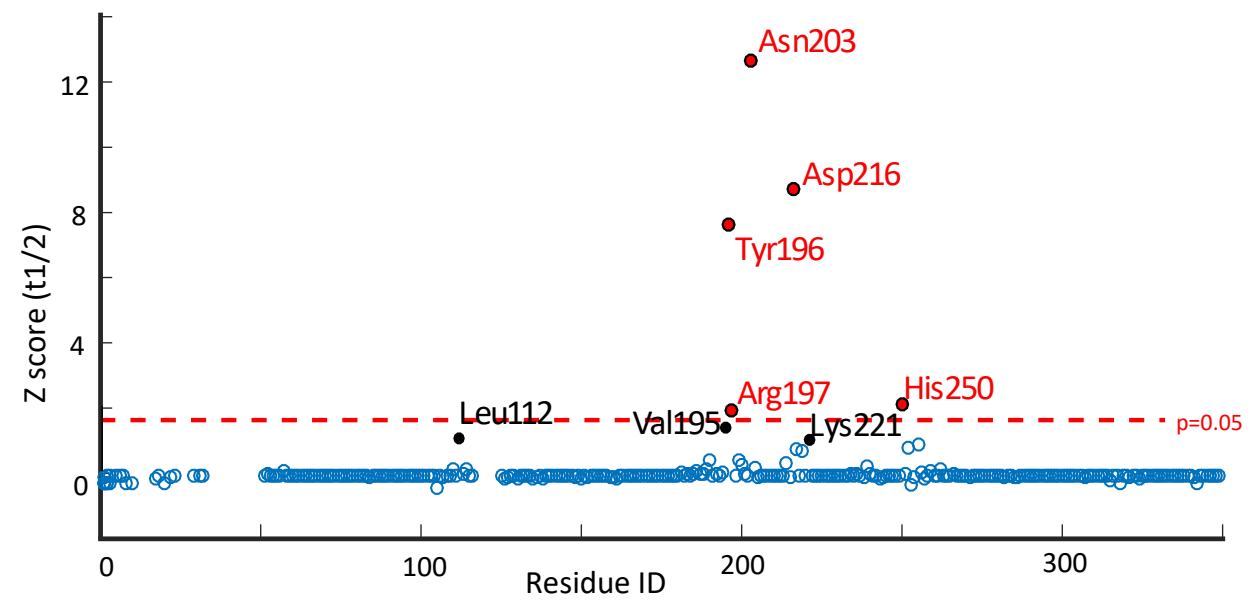

B.
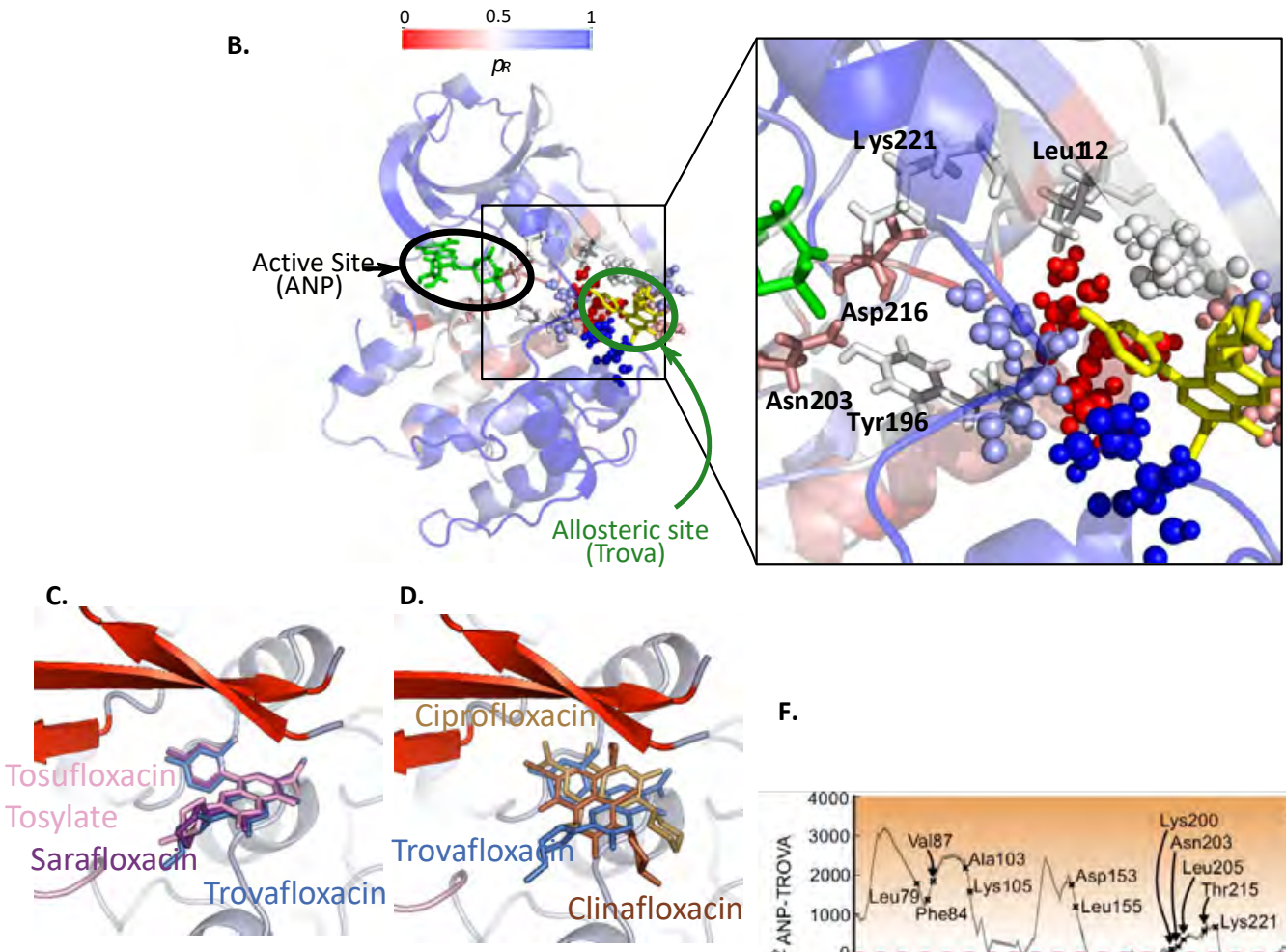

E.
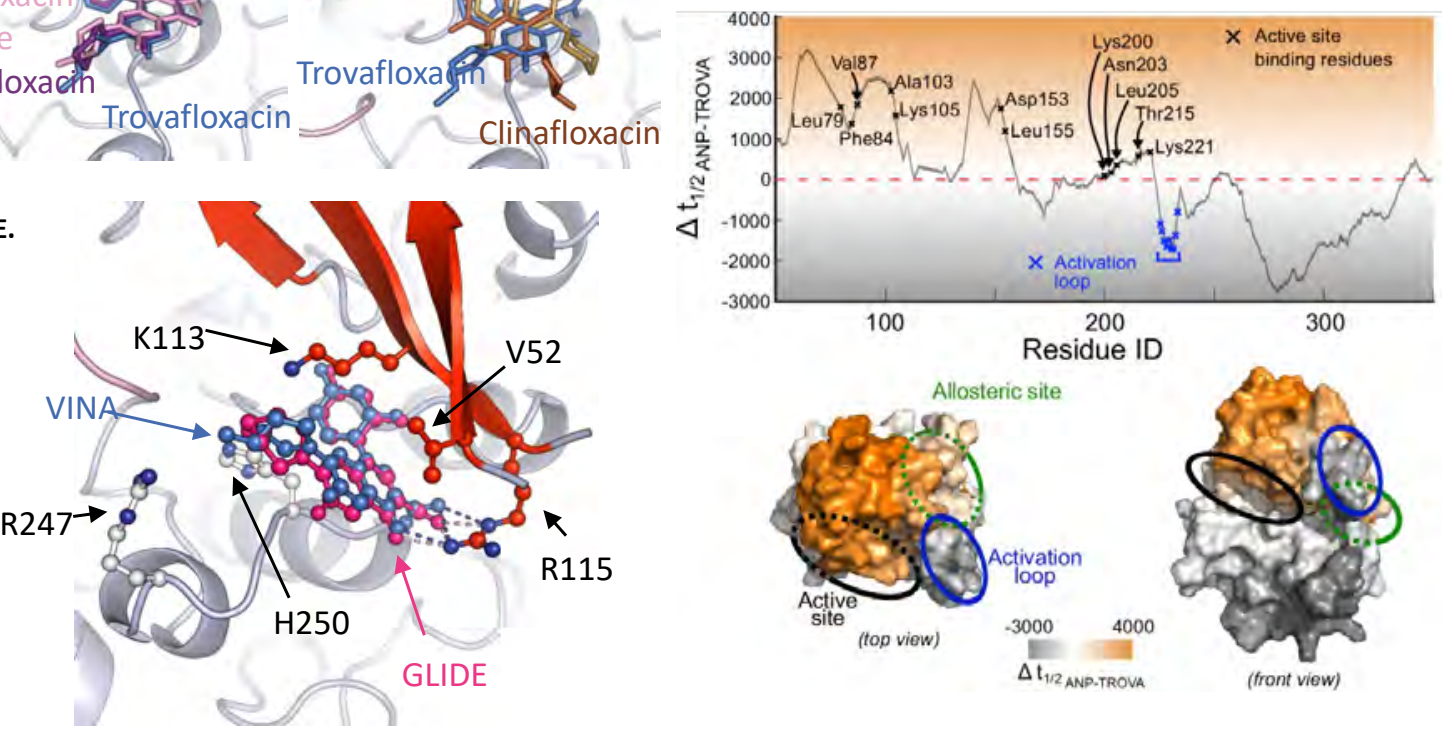

F.

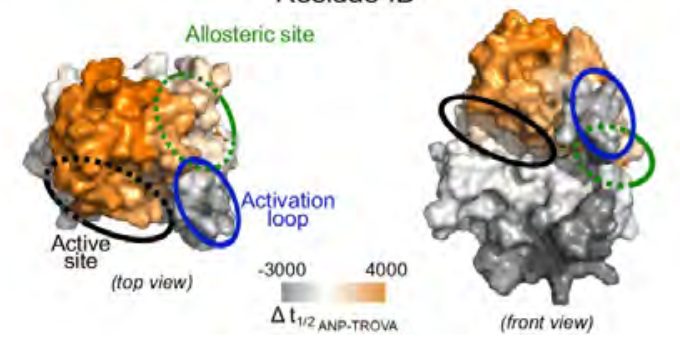

Fig. S13: Identifying mutations that perturb the communication between the active site and allosteric site through exhaustive computational alanisation. (A) An exhaustive computational alanine scanning of RSK4 is carried out by removing the weak interactions of each residue, one at a time. This computational alanine scanning identifies mutations that perturb strongly the communication between the active site and the allosteric site inducing large increases in the Markov 
transient time $t_{1 / 2}$. The alanisation of the highlighted residues (red, $\mathrm{Z}$ score $>1.64$, corresponding to $p<0.05$ ) induces the strongest reduction in the communication between the allosteric and active sites. (B) The active-site ligand (green sticks), the ligand Trova (yellow sticks) and the allosteric residues where TROVA binds (spheres) are shown on the structure of RSK4. Residues highly important under alanisation (high Z score in (A)) are shown as sticks and form two pathways, which connect the active site and allosteric site. (C) The top-ranked pose of Trovafloxacin, Tosufloxacin Tosylate and Sarafloxacin (positive controls) obtained using Autodock VINA are shown in marine blue, pink and purple respectively. (D) The top-ranked pose of Trovafloxacin, Ciprofloxacin and Clinafloxacin (negative controls) are shown in marine blue, pale-brown and brown respectively. (E) Docking of Trovafloxacin to the RSK4 NTKD structure. The top-ranked pose of Trovafloxacin obtained using Autodock VINA and GLIDE are shown in marine blue and hot-pink respectively. The Structure were superimposed using the RSK4 NTKD structure and oriented as in figure 6E. The $\beta B$-sheet is highlighted in red and the activation loop in pink. Key residues of the binding site are shown in balland-stick coloured by element. $\mathrm{H}$-bonds are identical in the two structures and are shown as blue and pink dotted lines respectively for the Autodock VINA and GLIDE outputs. (F) The difference in Markov transient times between the random walks originating at the AMP-PNP and Trovafloxacinbound allosteric sites $(\Delta \mathrm{t} 1 / 2=\mathrm{t} 1 / 2 \mathrm{ANP}-\mathrm{t} 1 / 2$ TROVA) is computed for all residues of the protein. As shown by the positive values of $\Delta \mathrm{t} 1 / 2>0$, the binding of trovafloxacin leads to increased communication with the residues of the active site (relative to AMP-PNP), whereas AMP-PNP exhibits increased communication with residues in the activation loop and lower lobe of RSK4 (relative to trovafloxacin), as indicated by $\Delta \mathrm{t} 1 / 2<0$. The value of $\Delta \mathrm{t} 1 / 2$ for each residue is mapped onto the protein structure (bottom). The active site and upper lobe are identified as regions better connected to the allosteric site after trovafloxacin binding. 

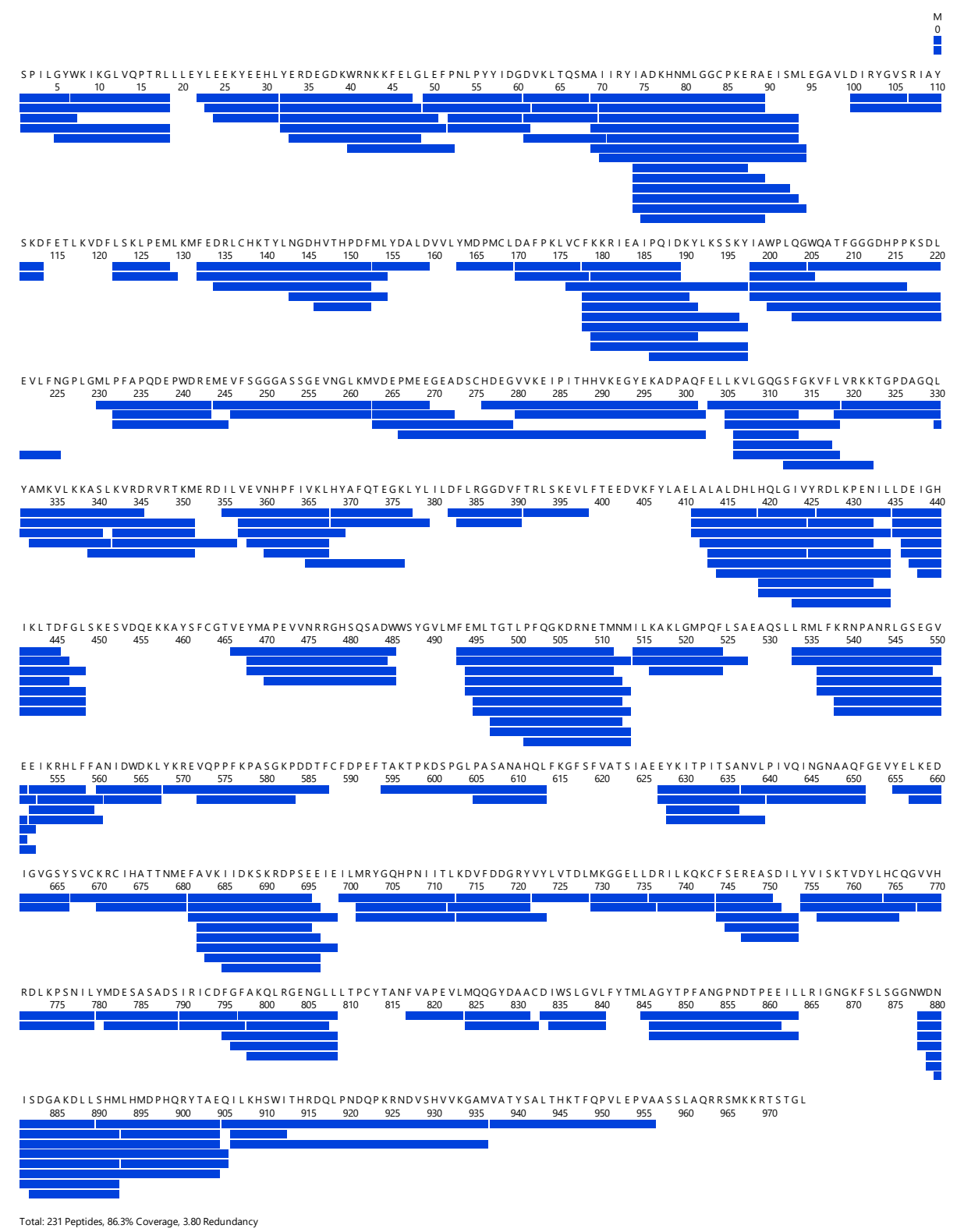

Total: 231 Peptides, $86.3 \%$ Coverage, 3.80 Redundancy

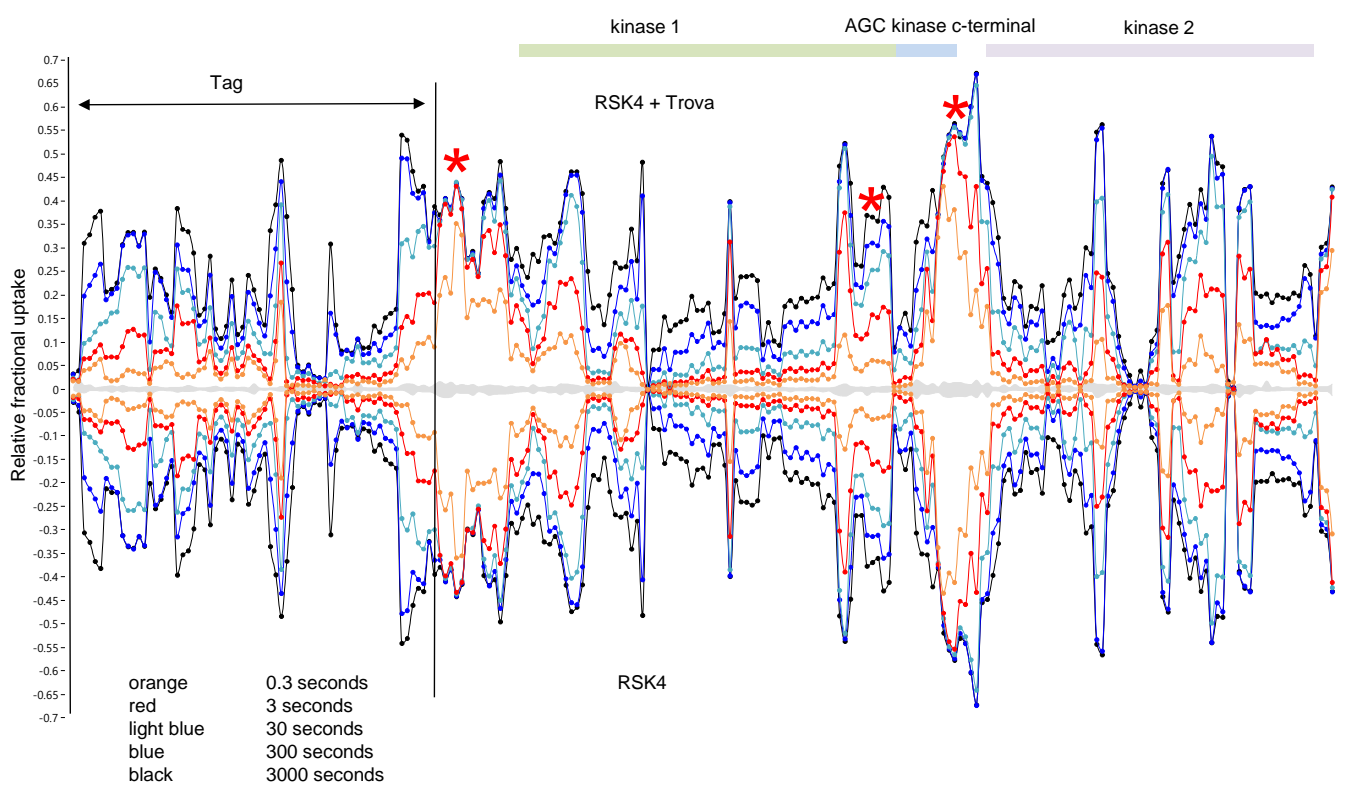


Fig. S14: Deuterium exchange mass-spectrometry. (A) Peptic peptide coverage of RSK4. (B) Butterfly plot displayed in the DynamX 3.0 software (Waters, UK) showing the incorporation of deuterium at successive time points indicated by the different colours as above. Relative fractional uptake is shown on the $y$-axis, without correction for back-exchange, while the experimental error is reported as a grey region on the $\mathrm{x}$-axis. 


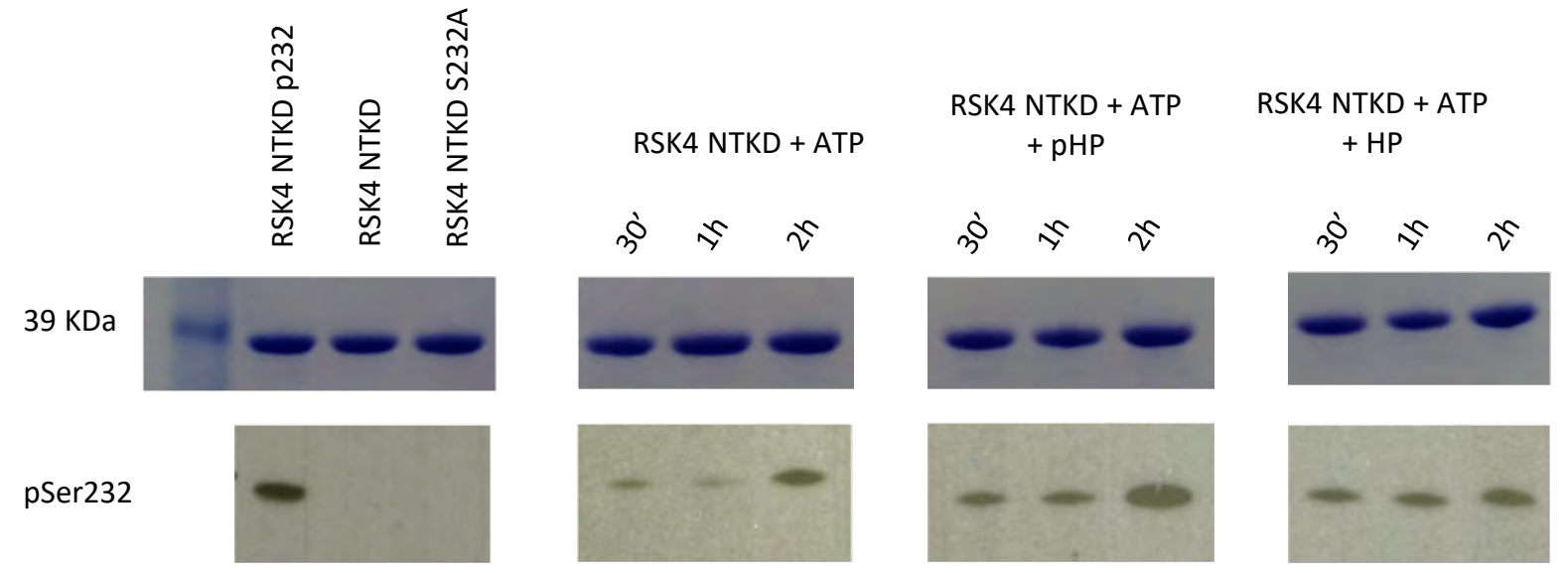

Fig. S15: The hydrophobic motif of RSK4 modulates the activity of the RSK4-NTKD. $10 \mu M$ RSK4 NTKD was incubated with $2 \mathrm{mM}$ ATP alone or in presence of $100 \mu \mathrm{M}$ of unphosphorylated (HP) or phosphorylated (pHP) hydrophobic motif peptide. Aliquots were collected after 30 minutes, 1 hour and 2 and phosphorylation of Ser 232 assessed by Western blotting. SDS-PAGE gels stained with Coomassie blue are shown on the top panel as loading control. On the left panels $10 \mu \mathrm{M}$ RSK4 NTKD pS232, RSK4 NTKD, RSK4 NTKD S232A were used respectively as positive control, time zero, and negative control. 
A.

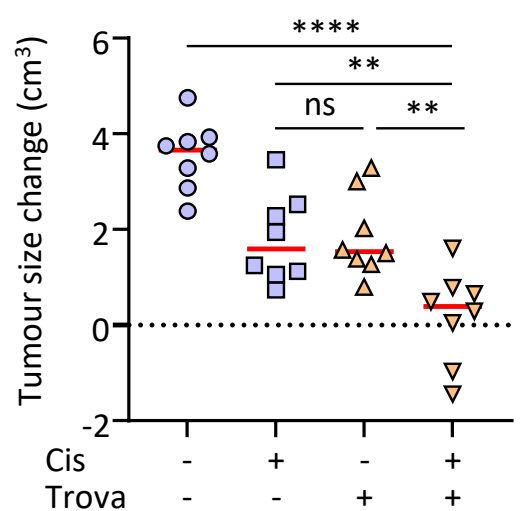

B.

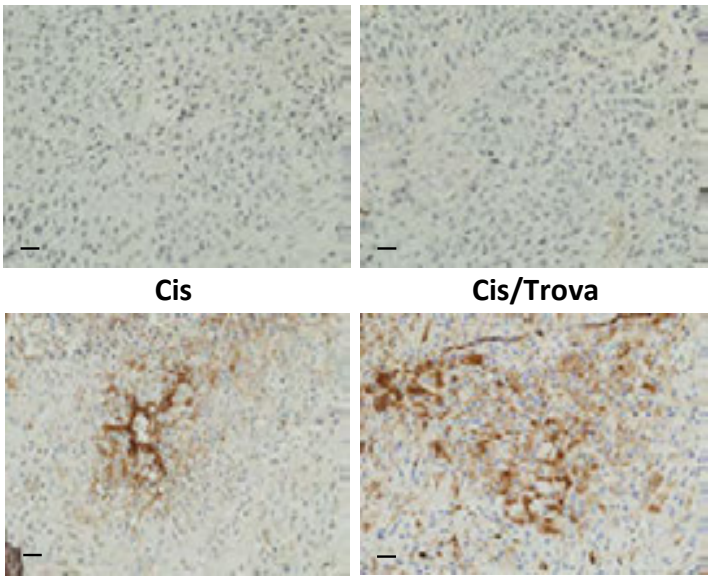

D.

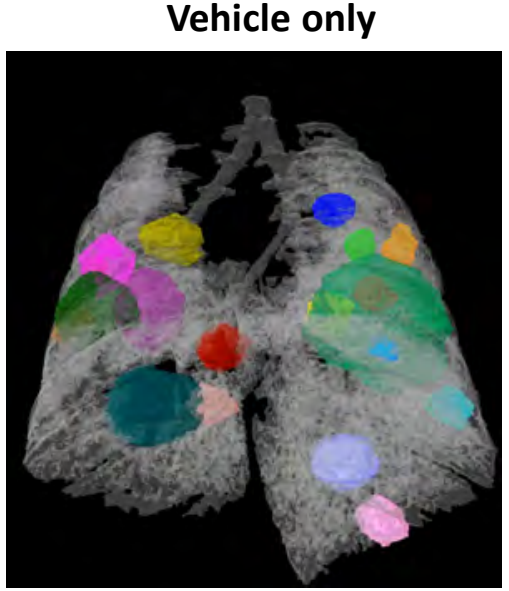

E.

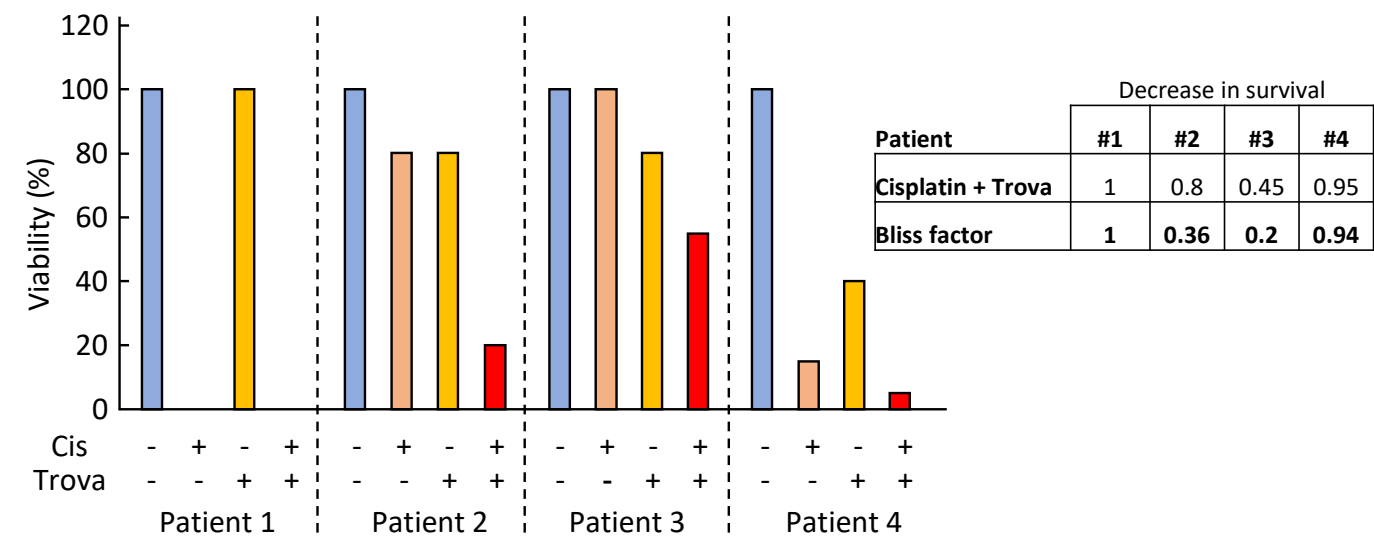

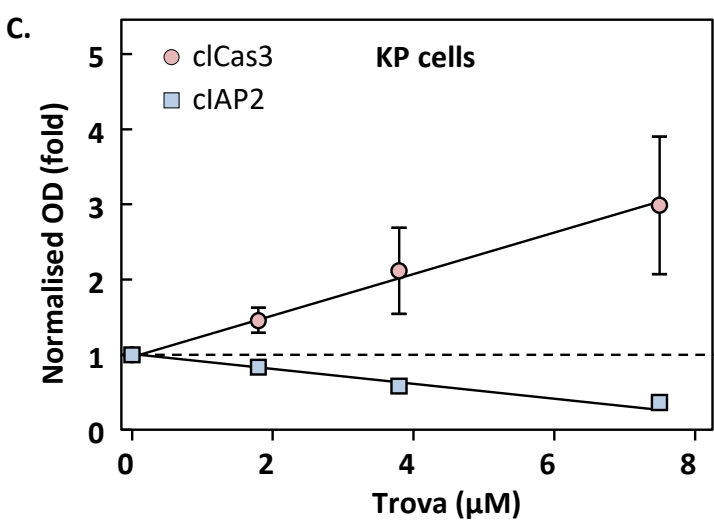

Trovafloxacin + Cisplatin

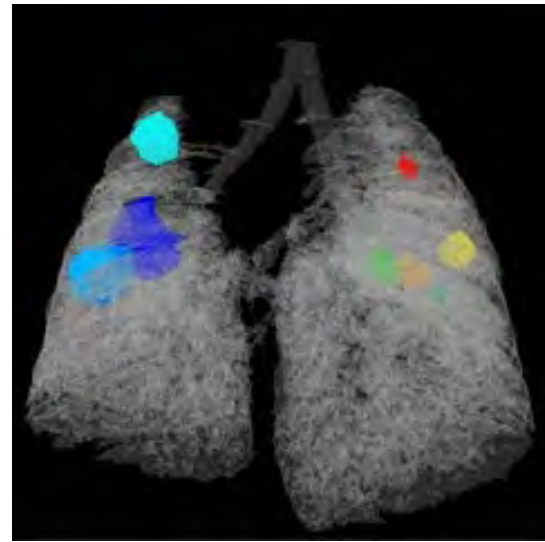

Patient 4 
Fig. S16: Trovafloxacin potentiates the effects of cisplatin treatment on tumours in vivo in mice and ex vivo on patient tumour explants. (A) Differences in tumour sizes corresponding to xenograft experiment presented in Fig 7A. Statistics: ANOVA with $* ; p<0.05, * * ; p<0.005, * * * * ; p \leq 0.001$. (B) Exemplar of caspase 3 cleavage staining (brown signal) obtained on FFPE A549 tumour samples from experiments presented as Fig 7A-B. Scale bar: $30 \mu \mathrm{m}$. (C) Optical densitometry of Western blotting $(n=3)$ for cIAP2 and cleaved caspase 3 from lysates of KP cells treated with a dose range of trovafloxacin. Signal was normalised to that for vinculin (loading control) and that of untreated condition. (D) Lung CT scans 3D reconstruction of vehicle-only and combination-treated KP mice. Exemplars animated 3D reconstructions of lung micro-CT scans for vehicle-only and trovafloxacin/cisplatin combination-treated KRASV12/TP53-/- (KP)-driven genetically-engineered mouse model from experiments shown as Fig 7D-F. Individual tractable tumours are alternatively coloured. Corresponding supplementary animation movies are provided (movies S1-S2). (E) Bladder cancer explants from 4 patients were treated with/without cisplatin $(40 \mu \mathrm{M})$ and trovafloxacin $(25$ $\mu \mathrm{M}$ ) alone or in combination and viability determined 5 days later as previously described (71). Lefthand table provides comparison between experimentally observed decrease in survival fraction in response to Cisplatin/Trovafloxacin combination and corresponding Bliss factor. 


\section{Consort diagram}

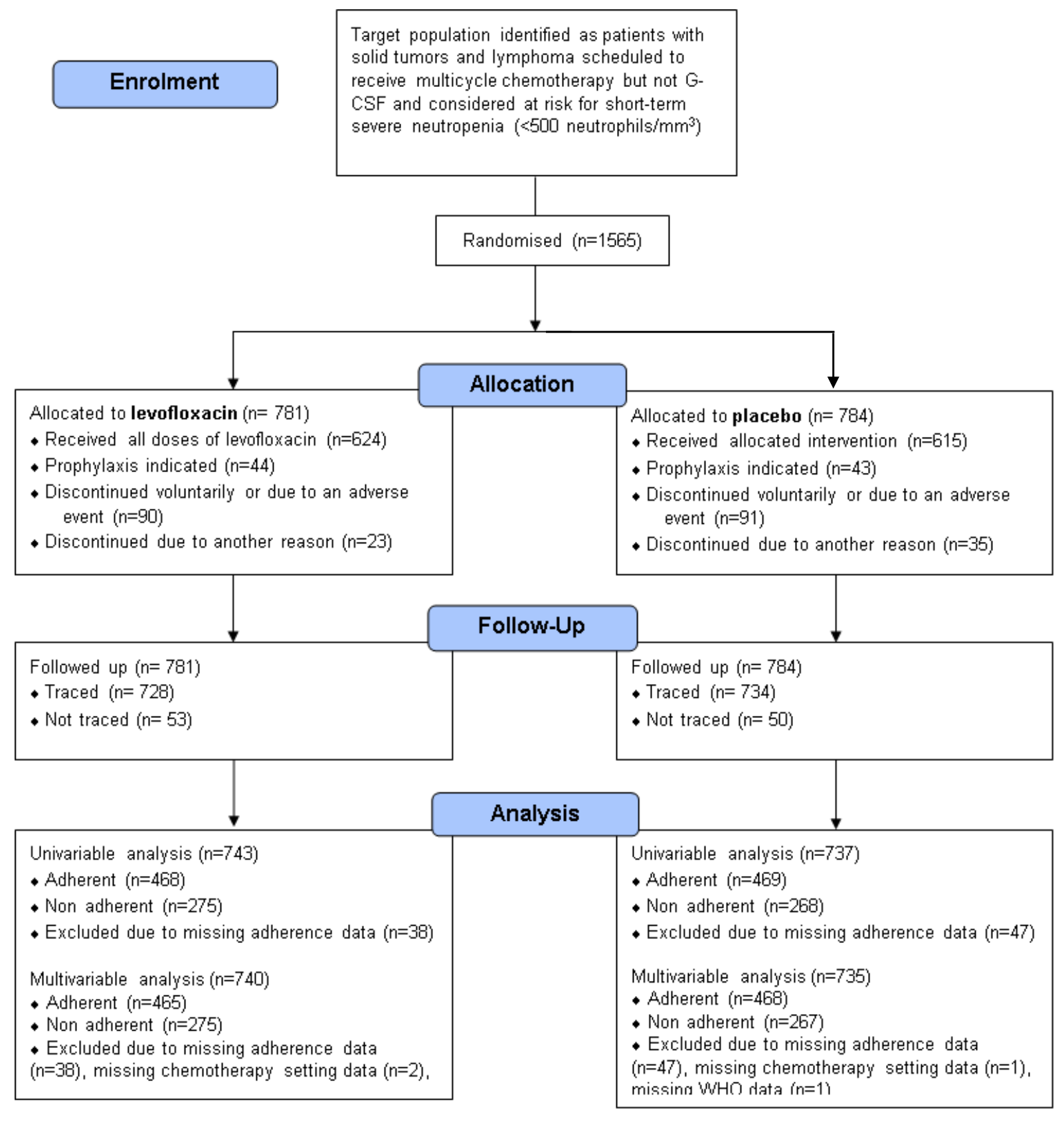

Fig. S17: CONSORT flow diagram of the SIGNIFICANT Trial. 
A.
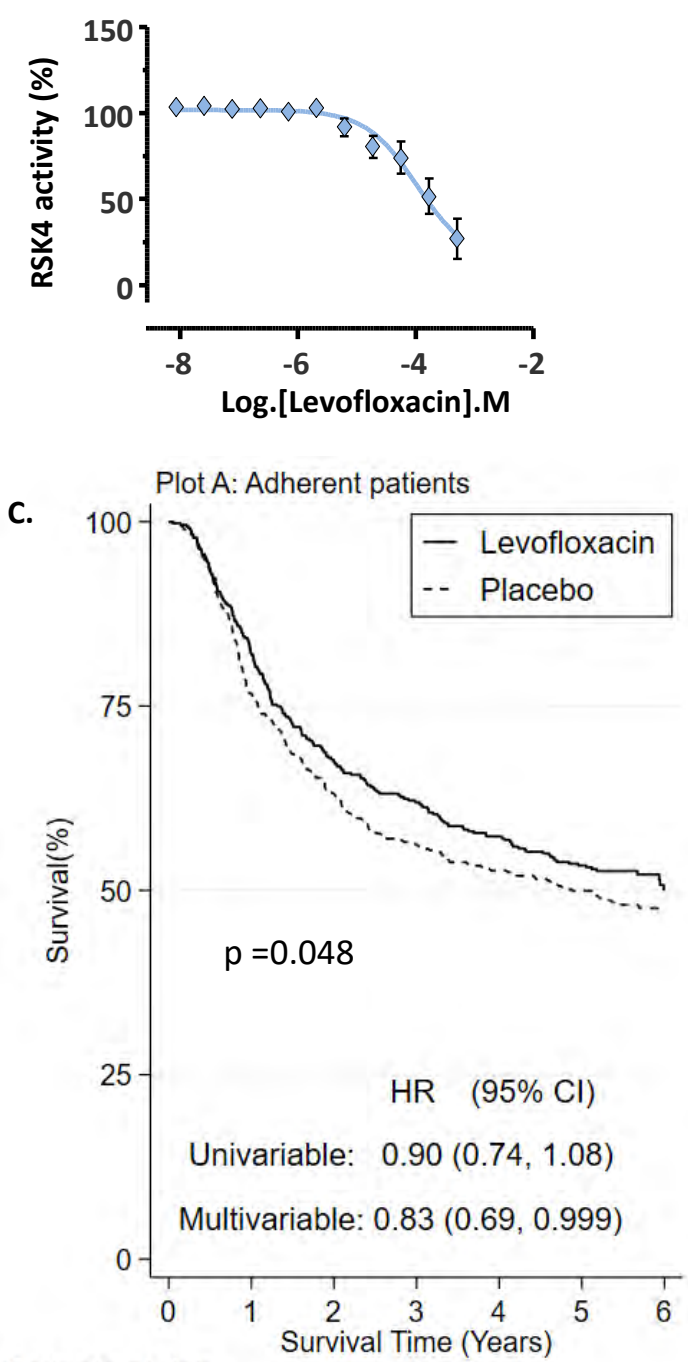

Patients at risk

$\begin{array}{llllllll}\text { Levo } & 468 & 354 & 291 & 267 & 247 & 228 & 64\end{array}$

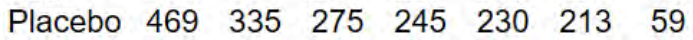

B.

Levo $(\mu \mathrm{M}) \quad 0 \quad 12.5 \quad 25 \quad 50100200$
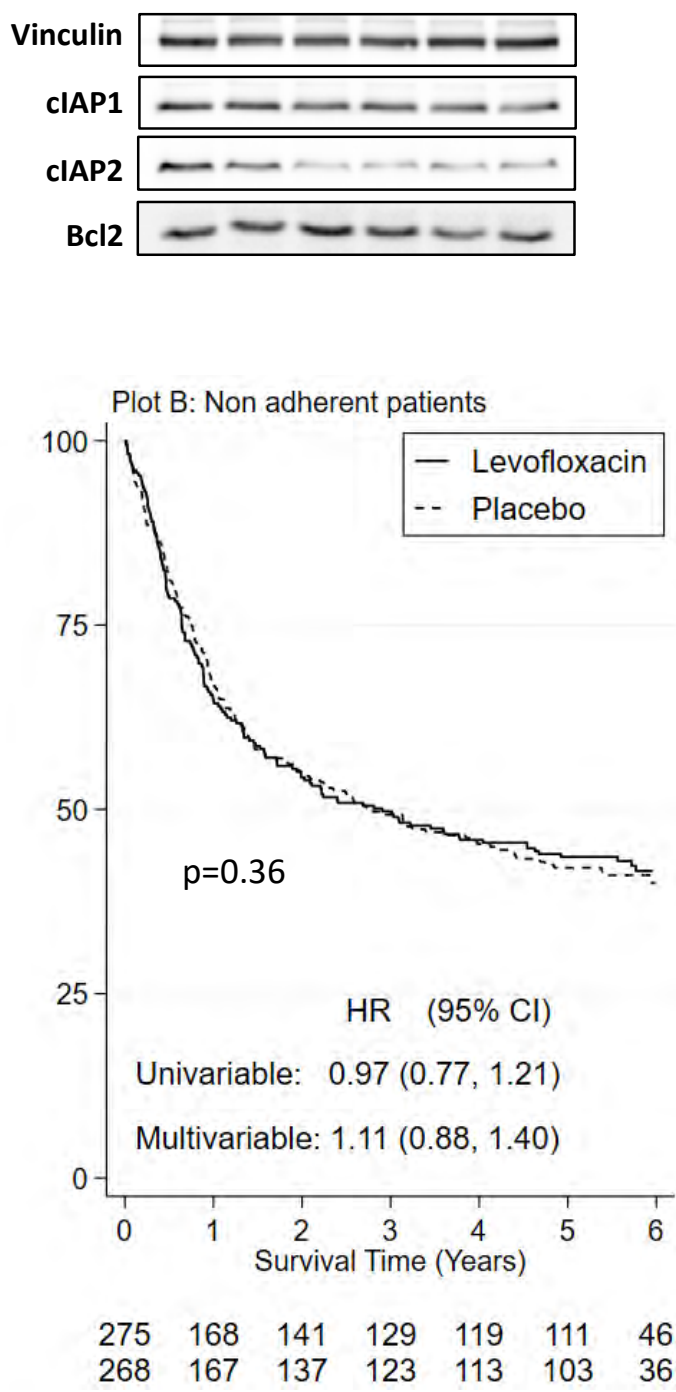

D.

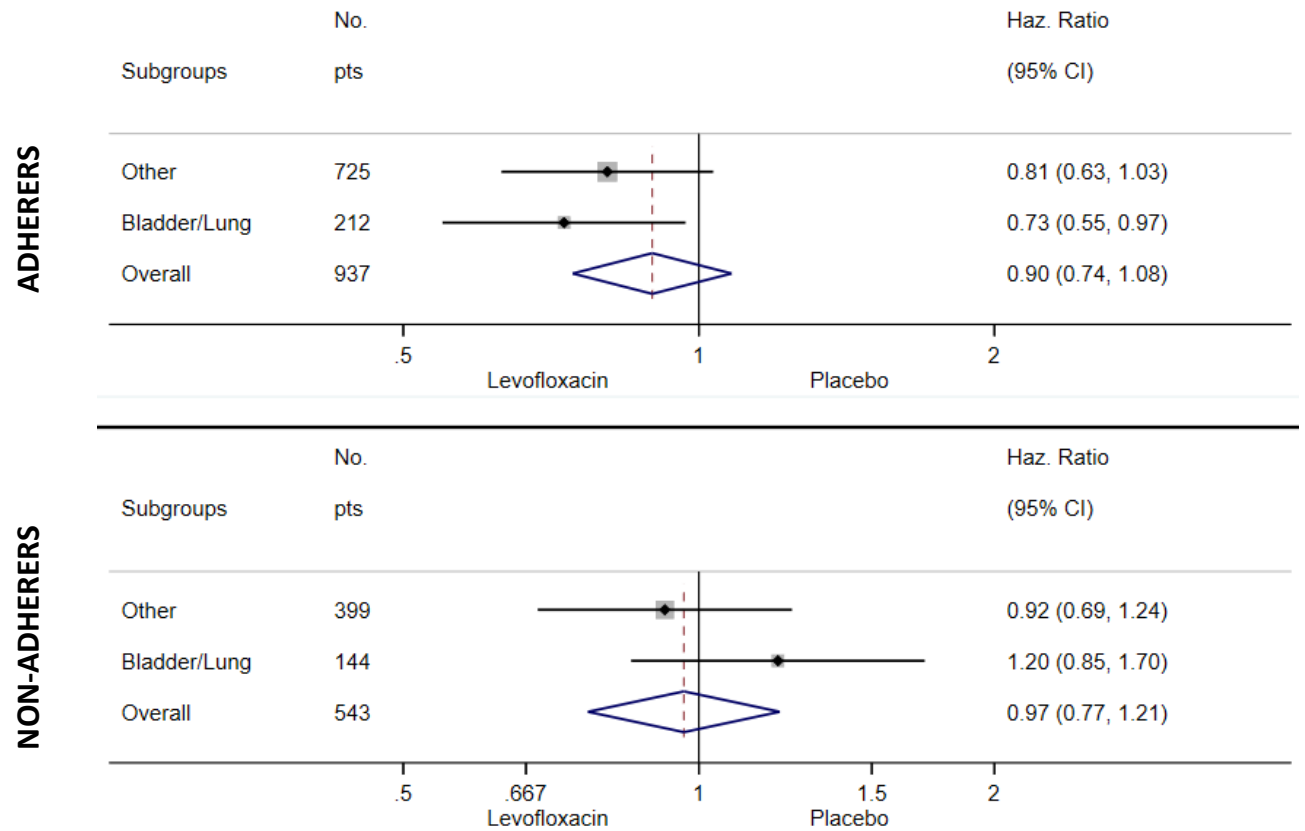


Fig. S18: Levofloxacin added to cisplatin-based chemotherapy improves survival in patients with cancers including lung and bladder. (A) Levofloxacin inhibits RSK4 activation by ERK2 in our HTRF assay. Data are representative of three replicate experiments and show mean \pm SEM of quadruplicates. (B) Lysates from A549 cells treated with or without levofloxacin (Levo) for $24 \mathrm{~h}$ were analysed by SDS-PAGE/Western blotting for the indicated proteins. Detection of vinculin was used as a loading control. (C) Kaplan-Meier overall survival curves of the SIGNIFICANT Trial comparing patients who were adherent (left panel) or non-adherent (right panel) in taking either levofloxacin (solid line) or placebo (dashed line) during chemotherapy for several cancer types including lung and bladder cancer. Hazard ratios (HR) are shown for univariate and multivariable analyses. (D) Forest plot of HR for levofloxacin-adherent and non-adherent lung and bladder cancer patients vs overall SIGNIFICANT cohort.

Table S1: Summary of data collection, structure determination, and refinement statistics for the RSK4-NTKD, RSK4-NTKD pS232 and RSK4-NTKD S232E crystals. 
RSK4 NTKD

RSK4 NTKD pS232

RSK4 NTKD S232E

\begin{tabular}{|c|c|c|c|c|c|}
\hline \multicolumn{6}{|c|}{ Data Collection } \\
\hline Space group & P 1211 & \multicolumn{2}{|l|}{ P 1211} & \multicolumn{2}{|l|}{ P 1211} \\
\hline Molecules ASU & 2 & \multicolumn{2}{|l|}{2} & \multicolumn{2}{|l|}{2} \\
\hline \multicolumn{6}{|l|}{ Cell dimensions } \\
\hline$a, b, c(\AA)$ & $113.92 \quad 62.18$ & 52.33 & 62.49 & 110.61 & 66.56 \\
\hline$\alpha, \beta, v\left({ }^{\circ}\right)$ & $\begin{array}{lll}90.00 & 90.24 & 90.00\end{array}$ & 90.00 & 90.00 & 90.22 & 90 \\
\hline Resolution (A) & $46.66-2.5(2.599-2.499)$ & \multicolumn{2}{|c|}{$47.67-3.0(3.107-3.0)$} & \multicolumn{2}{|c|}{$28.52-2.5(2.589-2.5)$} \\
\hline Redundancy & 1.9 & \multicolumn{2}{|l|}{2.0} & \multicolumn{2}{|c|}{3.3} \\
\hline $\mathrm{I} / \sigma$ & $9.31(2.75)$ & \multicolumn{2}{|l|}{$7.67(3.53)$} & \multicolumn{2}{|l|}{$2.36(2.51)$} \\
\hline $\mathrm{R}_{\text {merge }}$ & 6.5 & \multicolumn{2}{|l|}{4.5} & \multicolumn{2}{|l|}{2.5} \\
\hline Completeness (\%) & $96.17(96.98)$ & \multicolumn{2}{|l|}{$99.76(99.93)$} & \multicolumn{2}{|l|}{$99.84(99.92)$} \\
\hline \multicolumn{6}{|c|}{ Refinement } \\
\hline No. reflections (unique) & $45256(23781)$ & \multicolumn{2}{|l|}{$30046(15236)$} & \multicolumn{2}{|l|}{$59546(26061)$} \\
\hline $\mathrm{R}_{\text {work }}(\%)$ & 18.99 & \multicolumn{2}{|l|}{22.18} & \multicolumn{2}{|l|}{22.59} \\
\hline$R_{\text {free }}(\%)$ & 23.70 & \multicolumn{2}{|l|}{26.76} & 23.96 & \\
\hline $\mathrm{CC} 1 / 2$ & $0.992(0.828)$ & $0.995(0.923)$ & & $0.00515(0.0155)$ & \\
\hline Average $\mathrm{B}$, all atoms $\left(\AA^{2}\right)$ & 27.0 & 60 & & 37.3 & \\
\hline Number of Atoms (total) & 5104 & 4447 & & 4653 & \\
\hline Protein & 4716 & 4299 & & 4505 & \\
\hline Water molecules & 262 & 54 & & 86 & \\
\hline $\mathrm{Zn}$ & 2 & 1 & & 0 & \\
\hline AMP-PNP & 124 & 93 & & 62 & \\
\hline RMSD from ideal geometry & & & & & \\
\hline Bond lengths $(\AA)$ & 0.012 & 0.004 & & 0.004 & \\
\hline Bond angles $\left({ }^{\circ}\right)$ & 1.34 & 0.77 & & 0.81 & \\
\hline Ramachandran plot (\%) & & & & & \\
\hline Favored & 96 & 96 & & 96 & \\
\hline Disallowed & 0 & 0.18 & & 0.18 & \\
\hline Twin law & & $h,-k,-1$ & & $h,-k,-1$ & \\
\hline
\end{tabular}


Table S2: siRNA sequences used to target RSK4

\begin{tabular}{|l|l|l|l|}
\hline Gene & Name & siRNA sequence (5'-3') & \multirow{2}{*}{ Provider } \\
\hline RPS6KA6 & D_01 & GCAAATGTATTACCAATTG - Exon 14 & \\
\cline { 1 - 3 } RPS6KA6 & D_02 & GGACAACATCCCAACATTA - Exon 16 & \multirow{2}{*}{ Dharmacon } \\
\hline RPS6KA6 & D_03 & GGTGGAAACTGGGACAATA - Exon 20 & \\
\hline RPS6KA6 & D_04 & GTAGATATGTTTACCTTGT - Exon 17 & \\
\hline
\end{tabular}

\begin{tabular}{|l|l|}
\hline \multirow{2}{*}{ Table S3: RT-qPCR primers usedGene } & Primers sequence (5'-3') \\
\hline \multirow{2}{*}{ HPRT } & Fwd: TGCTCAAGGTTCTTGGTCAG (Exon 3) \\
\cline { 2 - 2 } & Rev: TTGTCCGACTCTGTCTCG (Exon 5) \\
\hline \multirow{2}{*}{ BCl-2 } & Fwd: TGACCTTGATTTATTTTGCATACC (Exon 2 \\
\cline { 2 - 2 } & Rev: CGAGCAAGACGTTCAGTCCT (Exon 3) \\
\hline \multirow{2}{*}{ PDCD4 } & Fwd: TTTGAGTTCGGTGGGGTCAT (Exon 1) \\
\cline { 2 - 2 } & Rev: TGACTTCACTTG TGGCCCAG (Exon 2) \\
\hline \multirow{2}{*}{ CIAP1 } & Fwd: GCAGAAAATGCTGGGACTGAG (Exon 4) \\
\cline { 2 - 2 } & Rev: TGTACCCCAGACACCTTTGC (Exon 5) \\
\hline \multirow{2}{*}{ CIAP2 } & Fwd: AGCTAGTCTGGGATCCACCTC \\
\cline { 2 - 2 } & Rev: GGGGTTAGTCCTCGATGAAG \\
\hline & Fwd: TGGAAGCTACCTCTCAGCCTAC \\
\cline { 2 - 2 } & Rev: GGAACTTCTCATCAAGGCAGA \\
\hline
\end{tabular}

\begin{tabular}{|l|l|l|l|l|l|}
\hline Protein & Species & MW (kDa) & Company & Cat. No. & Clone \\
\hline RSK4 & Rabbit & 84 & Abcam & Ab76117 & EP1982Y \\
\hline clAP1 & Rabbit & 72 & Abcam & Ab108361 & EPR4673 \\
\hline clAP2 & Rabbit & 72 & Cell Signalling & 3130 & 58C7 \\
\hline BCL2 & Mouse & 25 & Santa Cruz & Sc-509 & 100 \\
\hline E-Cadherin & Rabbit & 135 & Cell Signalling & 3195 & - \\
\hline N-Cadherin & Mouse & 135 & Cell Signalling & 14215 & 13 A9 \\
\hline Vimentin & Rabbit & 57 & Cell Signalling & 5741 & D21H3 \\
\hline PARP & Rabbit & 29 & Cell Signalling & 9542 & - \\
\hline Caspase 3 (Cleaved; Asp175) & Rabbit & 17,19 & Cell Signalling & 9661 & - \\
\hline Caspase 7 & Rabbit & 20,35 & Cell Signalling & 9492 & - \\
\hline NFkB 1 p105/P50 & Rabbit & 105,50 & Cell Signalling & 12540 & - \\
\hline NFkB p65 & Mouse & 65 & Cell Signalling & 6956 & L8F6 \\
\hline FLAG & Mouse & & Sigma & F1804 & M2 \\
\hline Vinculin & Mouse & 150 & Sigma & V9131 & hVIN-1 \\
\hline Tubulin & Mouse & 52 & Sigma & T5168 & B-5-1-2 \\
\hline Actin & Mouse & 42 & Sigma & A2228 & AC-74 \\
\hline Lamin B & Goat & 70 & Santa Cruz & sc-6216 & C-20 \\
\hline
\end{tabular}




\begin{tabular}{|l|l|l|l|l|l|}
\hline P300 & Mouse & 300 & Santa Cruz & sc-48343 & F-4 \\
\hline Phospho Ser 89-P300 & Rabbit & 300 & Santa Cruz & sc-130210 & - \\
\hline HSP90 & Mouse & 90 & Sigma & $05-594$ & - \\
\hline
\end{tabular}


The following data files are available in the online version of the supplement:

Data file S1. Trovafloxacin has no major activity against a panel of 140 recombinant kinases.

(Excel)

Movie S1. Micro-CT projection of lung of an exemplar vehicle-only treated KP mouse

Movie S2. Micro-CT projection of lung of an exemplar trovafloxacin/cisplatin combination treated KP mouse 